\title{
Chemoselective Formation of Successive Triazole Linkages in One Pot: 'Click-Click' Chemistry
}

\author{
Vincent Aucagne ${ }^{\star \dagger}$ and David A. Leigh* \\ School of Chemistry, University of Edinburgh, West Mains Road, Edinburgh EH9 3JJ, UK \\ aucagne@cnrs-orleans.fr; david.leigh@ed.ac.uk
}

\section{Experimental Methods}

General: Unless stated otherwise, all reagents and anhydrous solvents were purchased from Aldrich Chemicals and used without further purification. All reactions were carried out under a nitrogen inert athmospher. Alkyne $\mathbf{1}$ and azide $\mathbf{3}$ were prepared according to literature procedures. ${ }^{1}$ Column chromatography was carried out using Kiesegel C60 (Merck, Germany) as the stationary phase, and TLC was performed on precoated silica gel plates $(0.25 \mathrm{~mm}$ thick, $60 \mathrm{~F}_{254}$, Merck, Germany) and observed under UV light. ${ }^{1} \mathrm{H}$ and ${ }^{13} \mathrm{C}$ NMR spectra were recorded on Bruker AV250, AV400 or AV500 instruments, at a constant temperature of $25^{\circ} \mathrm{C}$. Chemical shifts are reported in parts per million from low to high field and referenced to TMS. Coupling constants $(J)$ are reported in hertz $(\mathrm{Hz})$. Standard abbreviations indicating multiplicity were used as follows: $\mathrm{m}=$ multiplet, $\mathrm{br}=$ broad, $\mathrm{d}=$ doublet, $\mathrm{q}=$ quadruplet, $\mathrm{t}=$ triplet, $\mathrm{s}=$ singlet. All melting points were determined using a Sanyo Gallenkamp apparatus and are reported uncorrected. Analytical RP-HPLC was performed using a Merck-Hitachi L7100 pump equiped with a C18 column, nucleosil $300 \AA(5 \mu \mathrm{m}, 250 \times 4.6 \mathrm{~mm})$, a L-7455 diode array detector and a Merck-Hitachi interface D-7000. Compounds were eluted with a linear gradient of $\mathrm{CH}_{3} \mathrm{CN} / \mathrm{H}_{2} \mathrm{O} / 0.1 \%$ TFA at $1 \mathrm{ml} / \mathrm{min}$ flow rate. Solvent A was water containing $0.1 \%$ TFA, solvent $\mathrm{B}$ was $\mathrm{CH}_{3} \mathrm{CN}$ containing $0.1 \%$ TFA. Gradient A : $25-55 \%$ of B over 30 min, then 55$95 \%$ of B over 3 min. Gradient B : $35-65 \%$ of B over 30 min then $65-95 \%$ of B over 3 min. The elution was followed at $214 \mathrm{~nm}$. Optical rotations were measured on a Perkin-Elmer 241 polarimeter. ESI mass spectrometry was performed with a Micromass Platform II Mass Spectrometer controlled using Masslynx v2.3 software while FAB mass spectrometry was carried out by the services at the University of Edinburgh. 


\section{1-(3-trimethylsilylprop-3-ynoxy)-4-(tris-(4-tert-butyl-phenyl)-methyl)-benzene (2)}

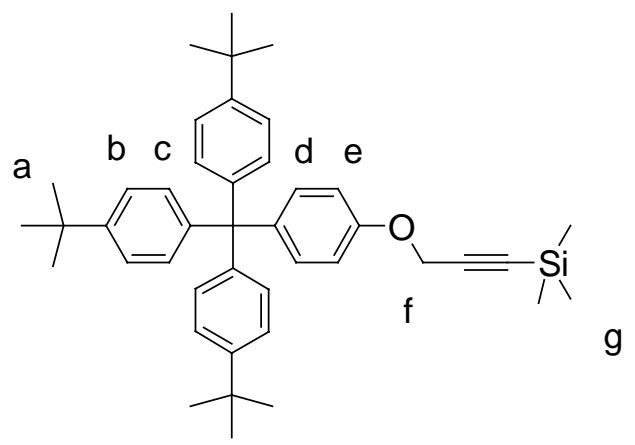

Alkyne $\mathbf{1}^{1}$ (123 mg, $\left.0.2 \mathrm{mmol}\right)$ was dissolved in THF (10 mL). The resulting solution was cooled down to $-40^{\circ} \mathrm{C}$ and $n$-BuLi $(138 \mu \mathrm{L}, 1.6 \mathrm{M}$ in hexanes, $0.22 \mathrm{mmol}, 1.1$ equiv) was added dropwise. After warming to $0^{\circ} \mathrm{C}$ and 15 min stirring, the solution was cooled to $-40^{\circ} \mathrm{C}$ and TMSCl $(31 \mu \mathrm{L}, 0.24 \mathrm{mmol}, 1.2$ equiv) was added. The reaction mixture was stirred at $\mathrm{rt}$ overnight, then quenched by an aqueous $\mathrm{NH}_{4} \mathrm{Cl}$ saturated solution $(10 \mathrm{~mL})$ and extracted with $\mathrm{CH}_{2} \mathrm{Cl}_{2}$ (4 x $10 \mathrm{~mL}$ ). Combined organic layers were dried over $\mathrm{MgSO}_{4}$ then filtrated and concentrated in vacuo. Resulting yellow amorphous solid was purified using flash column chromatography on silica gel (eluent: $\mathrm{CH}_{2} \mathrm{Cl}_{2}$ /petroleum ether gradient from 1/9 to 2/8) to give pure TMS-alkyne 2 as a colorless amorphous solid (104 mg, 85\%). ${ }^{1} \mathrm{H} \mathrm{NMR} \mathrm{(400} \mathrm{MHz}, \mathrm{CDCl}_{3}$, $298 \mathrm{~K}): \delta=0.17$ (s, 9H, Hg), 1.30 (s, 27H, Ha), 4.65 (s, 2H, Hf), 6.83 (d, 2H, J = 9.1 Hz, He), $7.07(\mathrm{~d}, 6 \mathrm{H}, J=8.6 \mathrm{~Hz}, \mathrm{Hb}), 7.10(\mathrm{~d}, 2 \mathrm{H}, J=9.1 \mathrm{~Hz}, \mathrm{Hd}), 7.23(\mathrm{~d}, 6 \mathrm{H}, J=8.6 \mathrm{~Hz}, \mathrm{Hc}) ;{ }^{13} \mathrm{C}$ NMR $\left(100 \mathrm{MHz}, \mathrm{CDCl}_{3}, 298 \mathrm{~K}\right): \delta=-0.1\left(\mathrm{CH}_{3}\right), 31.5\left(\mathrm{CH}_{3}\right), 34.5(\mathrm{C}), 57.0\left(\mathrm{CH}_{2}\right), 63.3(\mathrm{C}), 92.7$ (C), $100.5(\mathrm{C}), 113.7(\mathrm{CH}), 124.2(\mathrm{CH}), 130.9(\mathrm{CH}), 132.3(\mathrm{CH}), 140.5(\mathrm{C}), 144.2(\mathrm{C}), 148.5(\mathrm{C})$, 155.8 (C); LRFAB-MS (3-NOBA matrix): $\mathrm{m} / \mathrm{z}=614$ [M]+; HRFAB-MS (3-NOBA matrix): m/z $=614.39605$ (calcd. for $\left.\mathrm{C}_{43} \mathrm{H}_{54} \mathrm{OSi}, 614.39440\right)$.

\section{1-\{4-[Tris-(4-tert-butyl-phenyl)-methyl]-phenoxymethyl\}-3-(3-\{4-[tris-(4-tert-butyl-phenyl)- methyl]-phenoxy\}-propyl)-1H-[1,2,3]triazole (4)}

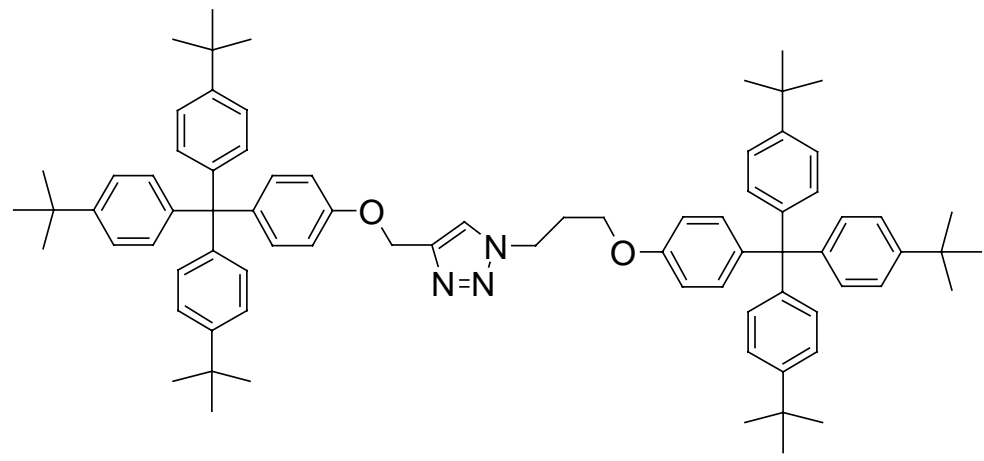

Method A: Alkyne 1 (10 mg, $0.018 \mathrm{mmol})$, TMS-alkyne 2 (11 mg, $0.018 \mathrm{mmol})$ and azide 3 (11 $\mathrm{mg}, 0.018 \mathrm{mmol})$ were dissolved in $4: 1 \mathrm{CH}_{2} \mathrm{Cl}_{2} / \mathrm{CH}_{3} \mathrm{OH}(500 \mu \mathrm{L})$ and $\mathrm{Cu}\left(\mathrm{CH}_{3} \mathrm{CN}\right)_{4} \mathrm{PF}_{6}(1.4 \mathrm{mg}$, 0.0036 mmol, 0.2 equiv) was added. The reaction was stirred at $\mathrm{rt}$ and monitored via ${ }^{1} \mathrm{H}$ NMR. 
Method B: TMS-Alkyne $2(11 \mathrm{mg}, 0.018 \mathrm{mmol})$ and azide $3(11 \mathrm{mg}, 0.018 \mathrm{mmol})$ were dissolved in 4:1 $\mathrm{CH}_{2} \mathrm{Cl}_{2} / \mathrm{CH}_{3} \mathrm{OH}(500 \mu \mathrm{L})$ and $\mathrm{Cu}\left(\mathrm{CH}_{3} \mathrm{CN}\right)_{4} \mathrm{PF}_{6}(1.4 \mathrm{mg}, 0.0036 \mathrm{mmol}, 0.2$ equiv) and $\mathrm{AgBF}_{4}(0.7 \mathrm{mg}, 0.018 \mathrm{mmol}, 0.2$ equiv) were added. The reaction was stirred at $\mathrm{rt}$ and monitored via ${ }^{1} \mathrm{H}$ NMR.

In both cases, after a few hours shaking at $\mathrm{rt}$, a new product was formed in near quantitative yield, whose ${ }^{1} \mathrm{H}$ NMR data were identical to those previously reported for $4 .{ }^{1}$

\section{1-(3-trimethylsilylprop-3-yne) 4-nitrophenyl carbonate}

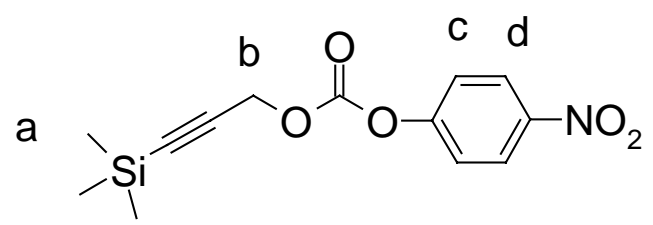

To an ice-cooled solution of 3-trimethylsilyl-prop-3-yn-1-ol (2.62 g, $16.9 \mathrm{mmol})$ in $\mathrm{CH}_{2} \mathrm{Cl}_{2}$ (100 $\mathrm{mL}$ ) and pyridine $(2.05 \mathrm{~mL}, 25.3 \mathrm{mmol}, 1.5$ equiv) was added 4-nitrophenyl chloroformate in one portion ( $3.40 \mathrm{~g}, 16.9 \mathrm{mmol}, 1$ equiv). The reaction mixture was stirred for $36 \mathrm{~h}$ at $\mathrm{rt}$, then cooled in an ice bath and quenched with a $1 \mathrm{M}$ aqueous $\mathrm{HCl}$ solution $(30 \mathrm{~mL})$ and extracted with $\mathrm{CH}_{2} \mathrm{Cl}_{2}$ $(2 \times 50 \mathrm{~mL})$. The combined organic layers were dried over $\mathrm{MgSO}_{4}$, filtered and concentrated in vacuo. The resulting yellow oil was purified using short-path flash column chromatography on silica gel (eluent: $\mathrm{CH}_{2} \mathrm{Cl}_{2}$ /petroleum ether gradient from $2 / 8$ to $\left.4 / 6\right)$ to give pure 1-(3trimethylsilylprop-3-yne), 4-nitrophenyl carbonate as a colorless oil (4.50 g, $91 \%) .{ }^{1} \mathrm{H}$ NMR $\left(400 \mathrm{MHz}, \mathrm{CDCl}_{3}, 298 \mathrm{~K}\right): \delta=0.20\left(\mathrm{~s}, 9 \mathrm{H}, \mathrm{Me}_{3} \mathrm{Si}\right), 4.88\left(\mathrm{~s}, 2 \mathrm{H}, \mathrm{CH}_{2} \mathrm{O}\right), 7.40(\mathrm{~d}, 2 \mathrm{H}, J=9.0 \mathrm{~Hz}$, $\left.\mathrm{H}_{\mathrm{Ar}}\right), 8.29\left(\mathrm{~d}, 2 \mathrm{H}, J=9.0 \mathrm{~Hz}, \mathrm{H}_{\mathrm{Ar}}\right) ;{ }^{13} \mathrm{C}$ NMR $\left(100 \mathrm{MHz}, \mathrm{CDCl}_{3}, 298 \mathrm{~K}\right): \delta=-0.5\left(\mathrm{CH}_{3}\right), 57.3$ $\left(\mathrm{CH}_{2}\right), 94.3(\mathrm{C}), 97.1(\mathrm{C}), 121.7(\mathrm{CH}), 125.3(\mathrm{CH}), 145.4(\mathrm{C}), 151.9(\mathrm{C}), 155.3(\mathrm{C})$; LRFAB-MS (3-NOBA matrix): $\mathrm{m} / \mathrm{z}=294[\mathrm{MH}]^{+}$; HRFAB-MS (3-NOBA matrix): $\mathrm{m} / z=294.07980$ (calcd. for $\mathrm{C}_{13} \mathrm{H}_{16} \mathrm{NO}_{5} \mathrm{Si}, 294.07978$ ).

\section{$N$-(3-Trimethylsilylprop-3ynoxycarbonyl)-L-phenylalanylglycylglycyne propargyl ester (5)}

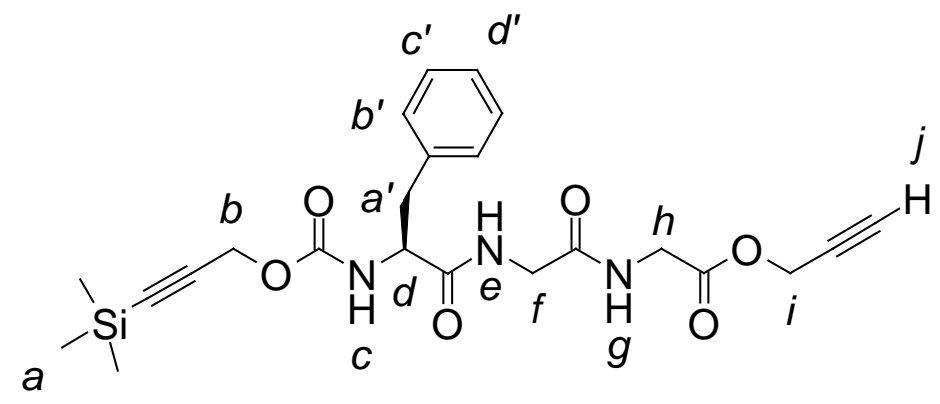

A solution of phenylalanylglycylglycine hydrochloride $(1 \mathrm{~g}, 3.58 \mathrm{mmol})$ in propargyl alcohol (40 $\mathrm{mL}$ ) was cooled in an ice bath. A $\mathrm{HCl}$ solution $\left(2 \mathrm{M}^{\text {in }} \mathrm{Et}_{2} \mathrm{O}, 9 \mathrm{~mL}, 17.9 \mathrm{mmol}, 5\right.$ equiv) was added and the reaction mixture stirred at $\mathrm{rt}$ for $18 \mathrm{~h}$ then concentrated in vacuo. Crude propargyl 
phenylalanylglycylglycinate hydrochloride (1.22 g) was dissolved in $\mathrm{CH}_{2} \mathrm{Cl}_{2}(40 \mathrm{~mL})$ and $\mathrm{Et}_{3} \mathrm{~N}$ (2.01 mL, $14.3 \mathrm{mmol}, 4$ equiv) and 1-(3-trimethylsilylprop-3-yne), 4-nitrophenyl carbonate (1.57 g, $5.37 \mathrm{mmol}, 1.5$ equiv) were added. The resulting yellow solution was stirred at $\mathrm{rt}$ for $18 \mathrm{~h}$ then quenched with a $1 \mathrm{M}$ aqueous $\mathrm{NaOH}$ solution $(20 \mathrm{~mL})$ and extracted with $\mathrm{CH}_{2} \mathrm{Cl}_{2}(2 \times 40 \mathrm{~mL})$. The combined organic layers were dried over $\mathrm{MgSO}_{4}$, filtered and concentrated in vacuo. The resulting yellow oil was purified using flash column chromatography on silica gel (eluent: $\mathrm{CH}_{2} \mathrm{Cl}_{2}$ then $\mathrm{CH}_{2} \mathrm{Cl}_{2} / \mathrm{MeOH}$ 98:2) to give pure dialkyne 5 as a colorless amorphous solid (1.38 g, $82 \%$ over two steps). $[\alpha]^{20}{ }_{\mathrm{D}}=+11.8\left(\mathrm{c} 1.0, \mathrm{CHCl}_{3}\right) ;{ }^{1} \mathrm{H} \mathrm{NMR}\left(400 \mathrm{MHz}, \mathrm{CDCl}_{3}, 298 \mathrm{~K}\right): \delta=$ $0.17\left(\mathrm{~s}, 9 \mathrm{H}, \mathrm{H}_{\mathrm{a}}\right), 2.51\left(\mathrm{t}, 1 \mathrm{H}, J=2.5 \mathrm{~Hz}, \mathrm{H}_{\mathrm{j}}\right), 3.06\left(\mathrm{dd}, 1 \mathrm{H}, J=7.1 \mathrm{~Hz}\right.$ and $\left.11.9 \mathrm{~Hz}, \mathrm{H}_{\mathrm{a}^{\prime} 1}\right), 3.12$ $\left(\mathrm{dd}, 1 \mathrm{H}, J=6.8 \mathrm{~Hz}\right.$ and $\left.11.9 \mathrm{~Hz}, \mathrm{H}_{\mathrm{a}}{ }^{2}\right), 3.91\left(\mathrm{dd}, 1 \mathrm{H}, J=5.7 \mathrm{~Hz}\right.$ and $\left.13.8 \mathrm{~Hz}, \mathrm{H}_{\mathrm{f} 1}\right), 3.97(\mathrm{dd}, 1 \mathrm{H}$, $J=5.6 \mathrm{~Hz}$ and $\left.13.8 \mathrm{~Hz}, \mathrm{H}_{\mathrm{f} 2}\right), 4.02\left(\mathrm{dd}, 1 \mathrm{H}, J=5.8 \mathrm{~Hz}\right.$ and $\left.14.4 \mathrm{~Hz}, \mathrm{H}_{\mathrm{h} 1}\right), 4.07(\mathrm{dd}, 1 \mathrm{H}, J=5.6 \mathrm{~Hz}$ and $\left.14.4 \mathrm{~Hz}, \mathrm{H}_{\mathrm{h} 2}\right), 4.36-4.44\left(\mathrm{~m}, 1 \mathrm{H}, \mathrm{H}_{\mathrm{d}}\right), 4.66\left(\mathrm{~s}, 2 \mathrm{H}, \mathrm{H}_{\mathrm{b}}\right), 4.74\left(\mathrm{~d}, 2 \mathrm{H}, J=2.5 \mathrm{~Hz}, \mathrm{H}_{\mathrm{i}}\right), 5.45(\mathrm{br}$ $\left.\mathrm{d}, 1 \mathrm{H}, J=6.8 \mathrm{~Hz}, \mathrm{H}_{\mathrm{c}}\right), 6.68-6.84\left(\mathrm{~m}, 2 \mathrm{H}, \mathrm{H}_{\mathrm{g}}\right.$ and $\left.\mathrm{H}_{\mathrm{e}}\right), 7.17-7.34\left(\mathrm{~m}, 5 \mathrm{H}, \mathrm{H}_{\mathrm{b}}, \mathrm{H}_{\mathrm{c}^{\prime}}\right.$ and $\left.\mathrm{H}_{\mathrm{d}^{\prime}}\right) ;{ }^{13} \mathrm{C}$ NMR (100 MHz, CDCl $3,298 \mathrm{~K}): \delta=-0.2\left(\mathrm{CH}_{3}\right), 38.1\left(\mathrm{CH}_{2}\right), 41.1\left(\mathrm{CH}_{2}\right), 43.1\left(\mathrm{CH}_{2}\right), 53.0\left(\mathrm{CH}_{2}\right)$, $54.0\left(\mathrm{CH}_{2}\right), 56.7(\mathrm{CH}), 75.7(\mathrm{CH}), 77.0(\mathrm{C}), 92.6(\mathrm{C}), 99.0(\mathrm{C}), 127.4(\mathrm{CH}), 129.0(\mathrm{CH}), 129.3$ (CH), 136.0 (C) 155.6 (C), 169.0 (C), 169.1 (C), 171.5 (C); LRFAB-MS (3-NOBA matrix): $m / z=$ $472[\mathrm{MH}]^{+}$; HRFAB-MS (3-NOBA matrix): $m / z=472.18935$ (calcd. for $\mathrm{C}_{23} \mathrm{H}_{30} \mathrm{~N}_{3} \mathrm{O}_{6} \mathrm{Si}$, 472.19039).

\section{General procedure for the formation of $N$-chloroacetyl aminoacid methyl esters:}

A solution of aminoacid methyl ester hydrochloride (1 mmol), chloroacetic acid (142 $\mathrm{mg}, 1.5$ mmol, 1.5 equiv), and DMAP (9 mg, $0.05 \mathrm{mmol}, 0.05$ equiv) in $\mathrm{CH}_{2} \mathrm{Cl}_{2}$ (10 mL) and triethylamine $\left(0.42 \mathrm{~mL}, 3 \mathrm{mmol}, 3\right.$ equiv) was cooled to $0^{\circ} \mathrm{C}$. EDCI $(288 \mathrm{mg}, 0.15 \mathrm{mmol}, 1.5$ equiv) was added and the solution stirred for $18 \mathrm{~h}$ at $\mathrm{rt}$ then quenched with water $(10 \mathrm{~mL})$ and diluted with $\mathrm{CH}_{2} \mathrm{Cl}_{2}(30 \mathrm{~mL})$. The organic layer was successively washed with $1 \mathrm{M}$ aqueous $\mathrm{HCl}$ $(2 \times 10 \mathrm{~mL}), 1 \mathrm{M}$ aqueous $\mathrm{NaHCO}_{3}(2 \times 10 \mathrm{~mL})$ and saturated aqueous $\mathrm{NaCl}(10 \mathrm{~mL})$. It was then dried over $\mathrm{MgSO}_{4}$, filtered and concentrated in vacuo. Resulting yellow oil was purified using flash column chromatography (eluent: $\mathrm{CH}_{2} \mathrm{Cl}_{2} / \mathrm{AcOEt} 8 / 2$ ) to give pure $N$-chloroacetyl aminoacid methyl esters 6a-d.

\section{$N$-Chloroacetyl-L-phenylalaninate methyl ester (6a)}<smiles>COC(=O)C([18O])NC(=O)CCl</smiles>

Obtained from commercial H-Phe-OMe.HCl (216 mg, $1 \mathrm{mmol})$ following the general procedure. Colorless oil (236 mg, $92 \%$ ). $[\alpha]^{20}{ }_{\mathrm{D}}=+45.0$ (c 1.0, $\mathrm{CHCl}_{3}$ ); ${ }^{1} \mathrm{H}$ NMR (400 MHz, $\mathrm{CDCl}_{3}, 298$ $\mathrm{K}): \delta=3.13\left(\mathrm{dd}, 1 \mathrm{H}, J=5.8 \mathrm{~Hz}\right.$ and $\left.13.8 \mathrm{~Hz}, \mathrm{H}_{\mathrm{a}^{\prime} 1}\right), 3.18\left(\mathrm{dd}, 1 \mathrm{H}, J=5.8 \mathrm{~Hz}\right.$ and $\left.13.8 \mathrm{~Hz}, \mathrm{H}_{\mathrm{a}^{\prime} 2}\right)$, $3.74\left(\mathrm{~s}, 3 \mathrm{H}, \mathrm{H}_{\mathrm{h}}\right), 4.01\left(\mathrm{~d}, 1 \mathrm{H}, J=15.1 \mathrm{~Hz}, \mathrm{H}_{\mathrm{a} 1}\right), 4.05\left(\mathrm{~d}, 1 \mathrm{H}, J=15.1 \mathrm{~Hz}, \mathrm{H}_{\mathrm{a} 2}\right), 4.83-4.91(\mathrm{~m}, 1 \mathrm{H}$, 
$\mathrm{H}_{\mathrm{c}}$ ), 6.90-7.02 (br d, $\left.1 \mathrm{H}, \mathrm{H}_{\mathrm{b}}\right), 7.08-7.35\left(\mathrm{~m}, 5 \mathrm{H}, \mathrm{H}_{\mathrm{b}}, \mathrm{H}_{\mathrm{c}}\right.$, and $\left.\mathrm{H}_{\mathrm{d}}\right) ;{ }^{13} \mathrm{C} \mathrm{NMR}\left(100 \mathrm{MHz}, \mathrm{CDCl}_{3}\right.$, $298 \mathrm{~K}): \delta=37.7\left(\mathrm{CH}_{2}\right), 42.3\left(\mathrm{CH}_{2}\right), 52.4\left(\mathrm{CH}_{3}\right), 53.3(\mathrm{CH}), 127.3(\mathrm{CH}), 128.6(\mathrm{CH}), 129.1(\mathrm{CH})$, $133.2(\mathrm{C}), 166.6(\mathrm{C}), 171.2(\mathrm{C})$; LRFAB-MS (3-NOBA matrix): $\mathrm{m} / z=256[\mathrm{MH}]^{+}$.

\section{$N$-Chloroacetyl-L-leucine methyl ester (6b)}

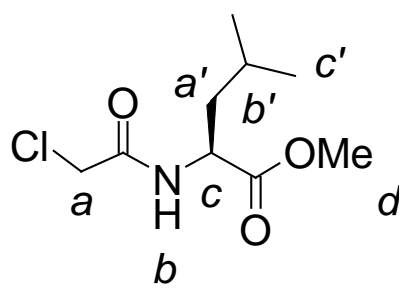

Obtained from commercial H-Leu-OMe. $\mathrm{HCl}(182 \mathrm{mg}, 1 \mathrm{mmol})$ following the general procedure. Colorless oil (208 mg, 94\%). [ $\alpha]^{20}{ }_{\mathrm{D}}=+5.0\left(\mathrm{c} 1.0, \mathrm{CHCl}_{3}\right) ;{ }^{1} \mathrm{H} \mathrm{NMR}\left(400 \mathrm{MHz}, \mathrm{CDCl}_{3}, 298 \mathrm{~K}\right): \delta$ $=0.95\left(\mathrm{~d}, 6 \mathrm{H}, J=6.1 \mathrm{~Hz}, \mathrm{H}_{\mathrm{c}^{\prime}}\right), 1.54-1.77\left(\mathrm{~m}, 3 \mathrm{H}, \mathrm{H}_{\mathrm{a}^{\prime}}\right.$ and $\left.\mathrm{H}_{\mathrm{b}^{\prime}}\right), 3.75\left(\mathrm{~s}, 3 \mathrm{H}, \mathrm{H}_{\mathrm{d}}\right), 4.07(\mathrm{~s}, 2 \mathrm{H}, \mathrm{Ha})$, 4.59-4.67 (m, 1H, $\left.\left.\mathrm{H}_{\mathrm{c}}\right), 6.80-6.97\left(\mathrm{br} \mathrm{d}, 1 \mathrm{H}, \mathrm{H}_{\mathrm{b}}\right) ;{ }^{13} \mathrm{C} \mathrm{NMR} \mathrm{(100} \mathrm{MHz,} \mathrm{CDCl} 3,298 \mathrm{~K}\right): \delta=22.9$ $\left(\mathrm{CH}_{3}\right), 24.9\left(\mathrm{CH}_{2}\right), 41.4\left(\mathrm{CH}_{2}\right), 42.5\left(\mathrm{CH}_{2}\right), 51.1(\mathrm{CH}), 52.6\left(\mathrm{CH}_{3}\right), 165.9(\mathrm{C}), 172.9(\mathrm{C})$; LRFAB-MS (3-NOBA matrix): $m / z=222[M H]^{+}$.

\section{$N$-Chloroacetyl-L-proline methyl ester (6c)}

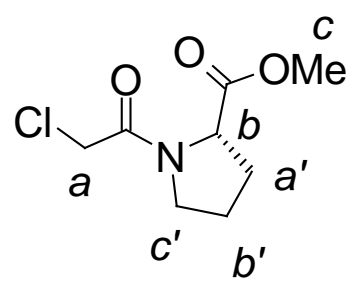

Obtained from commercial H-Pro-OMe. $\mathrm{HCl}(166 \mathrm{mg}, 1 \mathrm{mmol})$ following the general procedure. Colorless oil $(182 \mathrm{mg}, 89 \%)$. Colorless oil. $[\alpha]^{20}{ }_{\mathrm{D}}=+46.3\left(\mathrm{c} 1.0, \mathrm{CHCl}_{3}\right) ;{ }^{1} \mathrm{H}$ NMR $(400 \mathrm{MHz}$, $\left.\mathrm{CDCl}_{3}, 298 \mathrm{~K}\right)$ : mixture of 2 rotamers, major $(\mathrm{M})$ and minor $(\mathrm{m}) \delta=1.86-2.33\left(\mathrm{~m}, 4 \mathrm{H}, \mathrm{H}_{\mathrm{a}}\right.$, and $\left.\mathrm{H}_{\mathrm{b}^{\prime}}\right), 3.56-3.72\left(\mathrm{~m}, 2 \mathrm{H}, \mathrm{H}_{\mathrm{c}^{\prime}}\right), 3.73\left(\mathrm{~s}, 2.49 \mathrm{H}, \mathrm{H}_{\mathrm{c}(\mathrm{M})}\right), 3.77\left(\mathrm{~s}, 0.51 \mathrm{H}, \mathrm{H}_{\mathrm{c}(\mathrm{m})}\right), 3.93(\mathrm{~d}, 0.17 \mathrm{H}, J=$ $\left.12.6 \mathrm{~Hz}, \mathrm{H}_{\mathrm{a} 1(\mathrm{~m})}\right), 3.97\left(\mathrm{~d}, 0.17 \mathrm{H}, J=12.6 \mathrm{~Hz}, \mathrm{H}_{\mathrm{a} 2(\mathrm{~m})}\right), 4.04\left(\mathrm{~d}, 0.83 \mathrm{H}, J=12.6 \mathrm{~Hz}, \mathrm{H}_{\mathrm{a} 1(\mathrm{M})}\right), 4.09$ $\left(\mathrm{d}, 0.83 \mathrm{H}, J=12.6 \mathrm{~Hz}, \mathrm{H}_{\mathrm{al}(\mathrm{M})}\right), 4.51\left(\mathrm{dd}, 0.83 \mathrm{H}, J=4.0 \mathrm{~Hz}, 8.7 \mathrm{~Hz}, \mathrm{H}_{\mathrm{b}(\mathrm{M})}\right), 4.58(\mathrm{dd}, 0.17 \mathrm{H}, J=$ $\left.4.0 \mathrm{~Hz}, 7.6 \mathrm{~Hz}, \mathrm{H}_{\mathrm{b}(\mathrm{M})}\right) ;{ }^{13} \mathrm{C}$ NMR $\left(100 \mathrm{MHz}, \mathrm{CDCl}_{3}, 298 \mathrm{~K}\right)$ : mixture of 2 rotamers, major $(\mathrm{M})$ and minor $(\mathrm{m}) \delta=22.4\left(\mathrm{~m}, \mathrm{CH}_{2}\right), 25.0\left(\mathrm{M}, \mathrm{CH}_{2}\right), 29.2\left(\mathrm{M}, \mathrm{CH}_{2}\right), 31.4\left(\mathrm{~m}, \mathrm{CH}_{2}\right), 41.9\left(\mathrm{~m}, \mathrm{CH}_{2}\right)$, $42.0\left(\mathrm{M}, \mathrm{CH}_{2}\right), 47.1\left(\mathrm{~m}, \mathrm{CH}_{2}\right), 47.2\left(\mathrm{M}, \mathrm{CH}_{2}\right), 52.5(\mathrm{M}, \mathrm{CH}), 53.0(\mathrm{~m}, \mathrm{CH}), 59.3\left(\mathrm{M}, \mathrm{CH}_{3}\right), 59.5$ $\left(\mathrm{m}, \mathrm{CH}_{3}\right), 165.2(\mathrm{M}, \mathrm{C}), 165.3(\mathrm{~m}, \mathrm{C}), 172.2$ (m, C), 172.3 (M, C); LRFAB-MS (3-NOBA matrix): $m / z=206[M H]^{+}$. 


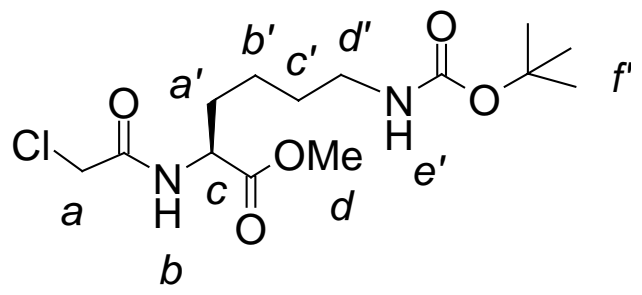

Obtained from commercial H-Lys(Boc)-OMe.HCl (297 mg, $1 \mathrm{mmol}$ ) following the general procedure. Colorless oil $(306 \mathrm{mg}, 91 \%)$. $[\alpha]^{20}{ }_{\mathrm{D}}=+22.5\left(\mathrm{c} 1.0, \mathrm{CHCl}_{3}\right) ;{ }^{1} \mathrm{H} \mathrm{NMR}(400 \mathrm{MHz}$, $\left.\mathrm{CDCl}_{3}, 298 \mathrm{~K}\right): \delta=1.20-1.52\left(\mathrm{~m}, 4 \mathrm{H}, \mathrm{H}_{\mathrm{b}}\right.$, and $\left.\mathrm{H}_{\mathrm{c}^{\prime}}\right), 1.39\left(\mathrm{~s}, 9 \mathrm{H}, \mathrm{H}_{\mathrm{f}}\right), 1.64-1.76\left(\mathrm{~m}, 1 \mathrm{H}, \mathrm{H}_{\mathrm{a}^{\prime} 1}\right)$, 1.80-1.91 (m, 1H, $\left.\mathrm{H}_{\mathrm{a}^{\prime} 2}\right), 2.99-3.11\left(\mathrm{~m}, 2 \mathrm{H}, \mathrm{H}_{\mathrm{d}^{\prime}}\right), 3.71\left(\mathrm{~s}, 3 \mathrm{~h} \mathrm{H}_{\mathrm{d}}\right), 4.03\left(\mathrm{~s}, 2 \mathrm{H}, \mathrm{H}_{\mathrm{a}}\right), 4.51-4.59$ (m, $\left.1 \mathrm{H}, \mathrm{H}_{\mathrm{c}}\right), 4.60-4.70\left(\mathrm{~m}, 1 \mathrm{H}, \mathrm{H}_{\mathrm{e}}\right), 7.10\left(\mathrm{Br} \mathrm{d}, \mathrm{H}, J=7.8 \mathrm{~Hz}, \mathrm{H}_{\mathrm{b}}\right) ;{ }^{13} \mathrm{C} \mathrm{NMR}\left(100 \mathrm{MHz}, \mathrm{CDCl}_{3}, 298\right.$ $\mathrm{K}): \delta=22.5\left(\mathrm{CH}_{2}\right), 28.4\left(\mathrm{CH}_{3}\right), 29.5\left(\mathrm{CH}_{2}\right), 31.8\left(\mathrm{CH}_{2}\right), 40.0\left(\mathrm{CH}_{2}\right), 42.4\left(\mathrm{CH}_{2}\right), 52.1\left(\mathrm{CH}_{3}\right)$, $52.6(\mathrm{CH}), 79.1(\mathrm{C}), 156.0$ (C) 165.9 (C), 172.2 (C); LRFAB-MS (3-NOBA matrix): $m / z=337$ $[\mathrm{MH}]^{+}$.

\section{General procedure for the formation of $\boldsymbol{N}$-azidoacetyl aminoacid methyl derivatives:}

A solution of $N$-chloroacetyl amino acid methyl ester 6a-d (1mmol) and $\mathrm{NaN}_{3}(135 \mathrm{mg}, 2 \mathrm{mmol}$, 2 equiv) in DMF (10 mL) was heated at $90^{\circ} \mathrm{C}$ for $18 \mathrm{~h}$. The solvent was removed in vacuo and the resulting yellow oil diluted with EtOAc $(40 \mathrm{~mL})$ then washed with water $(5 \times 5 \mathrm{~mL})$. The organic layer was dried over $\mathrm{MgSO}_{4}$, filtered and concentrated in vacuo. The resulting yellow oil was purified using flash column chromatography (eluent: $\mathrm{CH}_{2} \mathrm{Cl}_{2} / \mathrm{AcOEt} 8 / 2$ ) to give pure azidemodified dipeptide $\mathbf{7 a - d}$.

\section{$N$-Azidooacetyl-L-phenylalaninate metyl ester (7a)}<smiles>COC(=O)C(Cc1ccccc1)NC(=O)C[Hg]</smiles>

Obatained from $6 \mathbf{a}$ following the general procedure $(231 \mathrm{mg}, 88 \%)$ as a colorless amorphous solid. $[\alpha]_{\mathrm{D}}^{20}=+40.7$ (c 1.0, $\mathrm{CHCl}_{3}$ ); ${ }^{1} \mathrm{H} \mathrm{NMR}\left(400 \mathrm{MHz}, \mathrm{CDCl}_{3}, 298 \mathrm{~K}\right): \delta=3.08(\mathrm{dd}, 1 \mathrm{H}, J=$ $6.3 \mathrm{~Hz}$ and $\left.13.9 \mathrm{~Hz}, \mathrm{H}_{\mathrm{a}^{\prime} 1}\right), 3.15\left(\mathrm{dd}, 1 \mathrm{H}, J=6.3 \mathrm{~Hz}\right.$ and $\left.13.9 \mathrm{~Hz}, \mathrm{H}_{\mathrm{a}}{ }^{\prime}\right), 3.72\left(\mathrm{~s}, 3 \mathrm{H}, \mathrm{H}_{\mathrm{h}}\right), 3.87(\mathrm{~d}$, $\left.1 \mathrm{H}, J=16.4 \mathrm{~Hz}, \mathrm{H}_{\mathrm{a} 1}\right), 3.92\left(\mathrm{~d}, 1 \mathrm{H}, J=16.4 \mathrm{~Hz}, \mathrm{H}_{\mathrm{a} 2}\right), 4.83-4.90\left(\mathrm{~m}, 1 \mathrm{H}, \mathrm{H}_{\mathrm{c}}\right), 7.90-7.02(\mathrm{br} \mathrm{d}, 1 \mathrm{H}$, $\left.\mathrm{H}_{\mathrm{b}}\right), 7.08-7.35\left(\mathrm{~m}, 5 \mathrm{H}, \mathrm{H}_{\mathrm{b}}, \mathrm{H}_{\mathrm{c}}\right.$ and $\left.\mathrm{H}_{\mathrm{d}^{\prime}}\right) ;{ }^{13} \mathrm{C} \mathrm{NMR}\left(100 \mathrm{MHz}, \mathrm{CDCl}_{3}, 298 \mathrm{~K}\right): \delta=37.7\left(\mathrm{CH}_{2}\right)$, $42.3\left(\mathrm{CH}_{2}\right), 52.4\left(\mathrm{CH}_{3}\right), 53.3(\mathrm{CH}), 127.3(\mathrm{CH}), 128.6(\mathrm{CH}), 129.1(\mathrm{CH}), 133.2(\mathrm{C}), 166.6(\mathrm{C})$, 171.2 (C); LRFAB-MS (3-NOBA matrix): $\mathrm{m} / z=263[\mathrm{MH}]^{+}$; HRFAB-MS (3-NOBA matrix): $\mathrm{m} / z$ $=263.1476$ (calcd. for $\mathrm{C}_{12} \mathrm{H}_{15} \mathrm{~N}_{4} \mathrm{O}_{3}, 263.11442$ ). 


\section{$N$-Azidoacetyl-L-leucine methyl ester (7b)}<smiles>COC(=O)C(CC(C)C)NC(=O)C[Hg]</smiles>

Obtained from 6b following the general procedure. Colorless oil $(214 \mathrm{mg}, 94 \%) .{ }^{1} \mathrm{H}$ NMR $\left(\mathrm{CDCl}_{3}\right):[\alpha]^{20}{ }_{\mathrm{D}}=+2.6\left(\mathrm{c} 1.0, \mathrm{CHCl}_{3}\right) ;{ }^{1} \mathrm{H} \mathrm{NMR}\left(400 \mathrm{MHz}, \mathrm{CDCl}_{3}, 298 \mathrm{~K}\right): \delta=0.94(\mathrm{~d}, 6 \mathrm{H}, J=$ 6.1 Hz, $\left.\mathrm{H}_{\mathrm{c}^{\prime}}\right), 1.52-1.73\left(\mathrm{~m}, 3 \mathrm{H}, \mathrm{H}_{\mathrm{a}}\right.$ and $\mathrm{H}_{\mathrm{b}}$ ), $3.74\left(\mathrm{~s}, 3 \mathrm{H}, \mathrm{H}_{\mathrm{d}}\right), 4.02(\mathrm{~s}, 2 \mathrm{H}, \mathrm{Ha}), 4.61-4.69(\mathrm{~m}, 1 \mathrm{H}$, $\left.\mathrm{H}_{\mathrm{c}}\right)$, 6.55-6.70 (m, 1H, $\left.\mathrm{H}_{\mathrm{b}}\right) ;{ }^{13} \mathrm{C} \mathrm{NMR}\left(100 \mathrm{MHz}, \mathrm{CDCl}_{3}, 298 \mathrm{~K}\right): \delta=22.9\left(\mathrm{CH}_{3}\right), 24.9\left(\mathrm{CH}_{2}\right)$, $41.6\left(\mathrm{CH}_{2}\right), 50.7(\mathrm{CH}), 52.5\left(\mathrm{CH}_{3}\right), 52.6\left(\mathrm{CH}_{2}\right), 165.9(\mathrm{C}), 172.9(\mathrm{C})$; LRFAB-MS (3-NOBA matrix): $m / z=229[\mathrm{MH}]^{+}$; HRFAB-MS (3-NOBA matrix): $m / z=229.12931$ (calcd. for $\mathrm{C}_{9} \mathrm{H}_{17} \mathrm{~N}_{4} \mathrm{O}_{3}, 229.13007$ )

\section{$N$-Azidoacetyl-L-proline methyl ester (7c)}

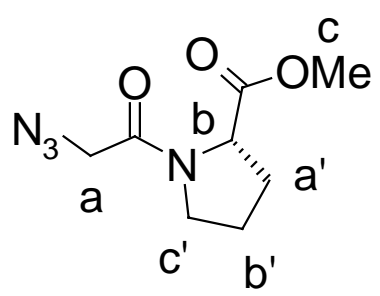

Obtained from $\mathbf{6 c}$ following the general procedure. Colorless oil $(182 \mathrm{mg}, 86 \%)$. $[\alpha]^{20}{ }_{\mathrm{D}}=+108.6$ (c 1.0, $\mathrm{CHCl}_{3}$ ); ${ }^{1} \mathrm{H} \mathrm{NMR}\left(400 \mathrm{MHz}, \mathrm{CDCl}_{3}, 298 \mathrm{~K}\right): 82: 18$ mixture of 2 rotamers, major (M) and minor $(\mathrm{m}) \quad \delta=1.83-2.33\left(\mathrm{~m}, 4 \mathrm{H}, \mathrm{H}_{\mathrm{a}}\right.$, and $\mathrm{H}_{\mathrm{b}}$ ) $), 3.40-3.49\left(\mathrm{~m}, 1 \mathrm{H}, \mathrm{H}_{\mathrm{cl}}\right.$ ) $), 3.54-3.72\left(\mathrm{~m}, 1 \mathrm{H}, \mathrm{H}_{\mathrm{c} 2^{\prime}}\right)$, $3.74\left(\mathrm{~s}, 2.46 \mathrm{H}, \mathrm{H}_{\mathrm{c}(\mathrm{M})}\right), 3.76\left(\mathrm{~s}, 0.54 \mathrm{H}, \mathrm{H}_{\mathrm{c}(\mathrm{m})}\right), 3.89\left(\mathrm{~s}, 2 \mathrm{H}, \mathrm{H}_{\mathrm{a}}\right), 4.36(\mathrm{dd}, 0.18 \mathrm{H}, J=3.2 \mathrm{~Hz}, 7.8$ $\left.\mathrm{Hz}, \mathrm{H}_{\mathrm{b}(\mathrm{m})}\right), 4.55\left(\mathrm{dd}, 0.82 \mathrm{H}, J=3.8 \mathrm{~Hz}, 8.6 \mathrm{~Hz}, \mathrm{H}_{\mathrm{b}(\mathrm{M})}\right) ;{ }^{13} \mathrm{C} \mathrm{NMR}\left(100 \mathrm{MHz}, \mathrm{CDCl}_{3}, 298 \mathrm{~K}\right)$ : mixture of 2 rotamers, major $(\mathrm{M})$ and minor $(\mathrm{m}) \delta=22.3\left(\mathrm{~m}, \mathrm{CH}_{2}\right), 24.9\left(\mathrm{M}, \mathrm{CH}_{2}\right), 29.1(\mathrm{M}$, $\left.\mathrm{CH}_{2}\right), 31.5\left(\mathrm{~m}, \mathrm{CH}_{2}\right), 46.4\left(\mathrm{M}, \mathrm{CH}_{2}\right), 47.0\left(\mathrm{~m}, \mathrm{CH}_{2}\right), 50.9\left(\mathrm{M}, \mathrm{CH}_{2}\right), 51.2\left(\mathrm{~m}, \mathrm{CH}_{2}\right), 52.6(\mathrm{M}$, $\mathrm{CH}), 53.0(\mathrm{~m}, \mathrm{CH}), 59.0\left(\mathrm{~m}, \mathrm{CH}_{3}\right), 59.1\left(\mathrm{M}, \mathrm{CH}_{3}\right), 166.3(\mathrm{M}, \mathrm{C}), 166.4(\mathrm{~m}, \mathrm{C}), 172.1(\mathrm{~m}, \mathrm{C})$, 172.3 (M, C); LRFAB-MS (3-NOBA matrix): $m / z=213[M H]^{+}$; HRFAB-MS (3-NOBA matrix): $m / z=213.09865$ (calcd. for $\mathrm{C}_{8} \mathrm{H}_{13} \mathrm{~N}_{4} \mathrm{O}_{3}, 213.09877$ ). 
$N^{\alpha}$-Azidoacetyl- $N^{\varepsilon}$-Boc-L-leucine methyl ester (7d)

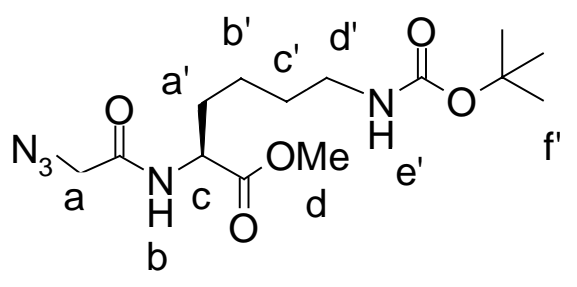

Obtained from 6d following the general procedure. Colorless oil (295 mg, 84\%). $[\alpha]^{20}{ }_{\mathrm{D}}=+14.4$ (c 1.0, $\left.\mathrm{CHCl}_{3}\right)$; ${ }^{1} \mathrm{H}$ NMR $\left(400 \mathrm{MHz}, \mathrm{CDCl}_{3}, 298 \mathrm{~K}\right): \delta=1.24-1.39\left(\mathrm{~m}, 2 \mathrm{H}, \mathrm{H}_{\mathrm{b}}\right), 1.43(\mathrm{~s}, 9 \mathrm{H}$, $\left.\mathrm{H}_{\mathrm{f}}\right)$, 1.44-1.55 (m, 2H, $\left.\mathrm{H}_{\mathrm{c}^{\prime}}\right)$, 1.67-1.79 (m, $\left.1 \mathrm{H}, \mathrm{H}_{\mathrm{a}^{\prime} 1}\right), 1.83-1.94\left(\mathrm{~m}, 1 \mathrm{H}, \mathrm{H}_{\mathrm{a}^{\prime} 2}\right), 3.04-3.16(\mathrm{~m}, 2 \mathrm{H}$, $\left.\mathrm{H}_{\mathrm{d}^{\prime}}\right), 3.76\left(\mathrm{~s}, 3 \mathrm{~h} \mathrm{H}_{\mathrm{d}}\right), 3.99$ (d, $\left.1 \mathrm{H}, J=16.6 \mathrm{~Hz}, \mathrm{H}_{\mathrm{a} 1}\right), 4.05\left(\mathrm{~d}, 1 \mathrm{H}, J=16.6 \mathrm{~Hz}, \mathrm{H}_{\mathrm{a} 1}\right), 4.50-4.65(\mathrm{~m}$, $2 \mathrm{H}, \mathrm{H}_{\mathrm{c}}$ and $\left.\mathrm{H}_{\mathrm{e}}\right), 6.81\left(\mathrm{br} \mathrm{d}, \mathrm{H}, J=7.8 \mathrm{~Hz}, \mathrm{H}_{\mathrm{b}}\right) ;{ }^{13} \mathrm{C} \mathrm{NMR}\left(100 \mathrm{MHz}, \mathrm{CDCl}_{3}, 298 \mathrm{~K}\right): \delta=22.5$ $\left(\mathrm{CH}_{2}\right), 28.5\left(\mathrm{CH}_{3}\right), 29.7\left(\mathrm{CH}_{2}\right), 32.0\left(\mathrm{CH}_{2}\right), 40.2\left(\mathrm{CH}_{2}\right), 52.0\left(\mathrm{CH}_{3}\right), 52.6\left(\mathrm{CH}_{2}\right), 52.7(\mathrm{CH}), 79.0$ (C), 156.2 (C) 166.6 (C), 172.4 (C); LRFAB-MS (3-NOBA matrix): $\mathrm{m} / z=344[\mathrm{MH}]^{+}$; HRFABMS (3-NOBA matrix): $m / z=344.19322$ (calcd. for $\mathrm{C}_{14} \mathrm{H}_{26} \mathrm{IN} \mathrm{O}_{5}, 344.19340$ ).

\section{TMS-alkyne triazole pseudohexapeptide (8)}

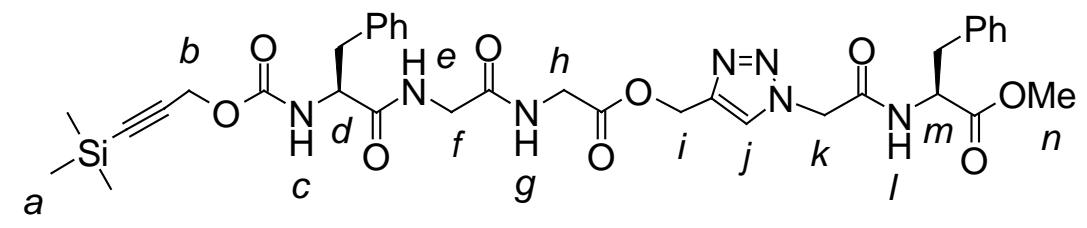

To a solution of dialkyne $5(47 \mathrm{mg}, 0.1 \mathrm{mmol})$ and azide-modified dipeptide $7 \mathbf{a}$ ( $26 \mathrm{mg}, 0.1$ mmol, 1.0 equiv) in $t$-BuOH $(4 \mathrm{~mL})$ and water $(200 \mu \mathrm{L})$ were added $\mathrm{CuSO}_{4}(0.1 \mathrm{M}$ in water, 100 $\mu \mathrm{L}, 0.01 \mathrm{mmol}, 0.1$ equiv) and sodium ascorbate $(0.1 \mathrm{M}$ in water, $200 \mu \mathrm{L}, 0.02 \mathrm{mmol}, 0.2$ equiv). The resulting solution was stirred at $35^{\circ} \mathrm{C}$ for $18 \mathrm{~h}$ then diluted with water $(10 \mathrm{~mL})$ and extracted with $\mathrm{CH}_{2} \mathrm{Cl}_{2}$ (4 x $10 \mathrm{~mL}$ ). The combined organic layers were dried over $\mathrm{MgSO}_{4}$, filtered and concentrated in vacuo. The resulting yellow oil was purified using short path flash column chromatography on silica gel (eluent: $\mathrm{CH}_{2} \mathrm{Cl}_{2} / \mathrm{MeOH}$ gradient from 98/2 to 90/10) to give pure triazole 8 as a colorless amorphous solid $(69 \mathrm{mg}, 94 \%)$. $[\alpha]^{20}{ }_{\mathrm{D}}=+21.3\left(\mathrm{c} 1.0, \mathrm{CHCl}_{3}\right) ;{ }^{1} \mathrm{H}$ NMR $\left(400 \mathrm{MHz}, \mathrm{CDCl}_{3}, 298 \mathrm{~K}\right): \delta=0.15\left(\mathrm{~s}, 9 \mathrm{H}, \mathrm{H}_{\mathrm{a}}\right), 2.96-3.17\left(\mathrm{~m}, 4 \mathrm{H}, \mathrm{CH}_{2} \mathrm{Ph}\right), 3.71\left(\mathrm{~s}, 3 \mathrm{H}, \mathrm{H}_{\mathrm{n}}\right), 3.82$ $\left(\mathrm{dd}, 1 \mathrm{H}, J=5.7 \mathrm{~Hz}\right.$ and $\left.16.7 \mathrm{~Hz}, \mathrm{H}_{\mathrm{fl}}\right), 3.88-4.01\left(\mathrm{~m}, 3 \mathrm{H}, \mathrm{H}_{\mathrm{f} 2}\right.$ and $\left.\mathrm{H}_{\mathrm{h}}\right), 4.38-4.46\left(\mathrm{~m}, 1 \mathrm{H}, \mathrm{H}_{\mathrm{d}}\right), 4.58$ $\left(\mathrm{d}, 1 \mathrm{H}, J=15.9 \mathrm{~Hz}, \mathrm{H}_{\mathrm{b} 1}\right), 4.63\left(\mathrm{~d}, 1 \mathrm{H}, J=15.9 \mathrm{~Hz}, \mathrm{H}_{\mathrm{b} 2}\right), 4.80-4.87\left(\mathrm{~m}, 1 \mathrm{H}, \mathrm{H}_{\mathrm{m}}\right), 4.98(\mathrm{~d}, 1 \mathrm{H}, J=$ $\left.16.2 \mathrm{~Hz}, \mathrm{H}_{\mathrm{k} 1}\right), 5.04\left(\mathrm{~d}, 1 \mathrm{H}, J=16.2 \mathrm{~Hz}, \mathrm{H}_{\mathrm{k} 2}\right), 5.24\left(\mathrm{~s}, 2 \mathrm{H}, \mathrm{H}_{\mathrm{i}}\right), 5.72\left(\mathrm{br} \mathrm{d}, 1 \mathrm{H}, J=7.3 \mathrm{~Hz}, \mathrm{H}_{\mathrm{c}}\right.$ ), $6.92\left(\mathrm{br} \mathrm{d}, 1 \mathrm{H}, J=7.4 \mathrm{~Hz}, \mathrm{H}_{\mathrm{l}}\right), 6.96-7.31\left(\mathrm{~m}, 12 \mathrm{H}, \mathrm{H}_{\mathrm{Ar}}, \mathrm{H}_{\mathrm{g}}\right.$ and $\left.\mathrm{H}_{\mathrm{e}}\right), 7.74\left(\mathrm{~s}, 1 \mathrm{H}, \mathrm{H}_{\mathrm{j}}\right) ;{ }^{13} \mathrm{C} \mathrm{NMR}$ $\left(100 \mathrm{MHz}, \mathrm{CDCl}_{3}, 298 \mathrm{~K}\right): \delta=-0.2\left(\mathrm{CH}_{3}\right), 37.7\left(\mathrm{CH}_{2}\right), 38.2\left(\mathrm{CH}_{2}\right), 41.4\left(\mathrm{CH}_{2}\right), 43.2\left(\mathrm{CH}_{2}\right), 52.7$ $\left(\mathrm{CH}_{2}\right), 52.8(\mathrm{CH}), 53.7(\mathrm{CH}), 53.9\left(\mathrm{CH}_{2}\right), 56.6\left(\mathrm{CH}_{3}\right), 58.3\left(\mathrm{CH}_{2}\right), 92.5(\mathrm{C}), 99.2(\mathrm{C}), 125.7(\mathrm{CH})$, $127.3(\mathrm{CH}), 127.4(\mathrm{CH}), 128.8(\mathrm{CH}), 128.9(\mathrm{CH}), 129.3(\mathrm{CH}), 129.4(\mathrm{CH}), 135.6(\mathrm{C}), 136.3(\mathrm{C})$, 142.8 (C), 155.6 (C), 165.0 (C), 169.4 (C), 169.5 (C), 171.7 (C), 171.8 (C); LRFAB-MS (3- 
NOBA matrix): $m / z=734[M H]^{+}$; HRFAB-MS (3-NOBA matrix): $m / z=734.29618$ (calcd. for $\left.\mathrm{C}_{35} \mathrm{H}_{44} \mathrm{~N}_{7} \mathrm{O}_{9} \mathrm{Si}, 734.29698\right)$.

\section{Alkyne triazole pseudohexapeptide (9)}

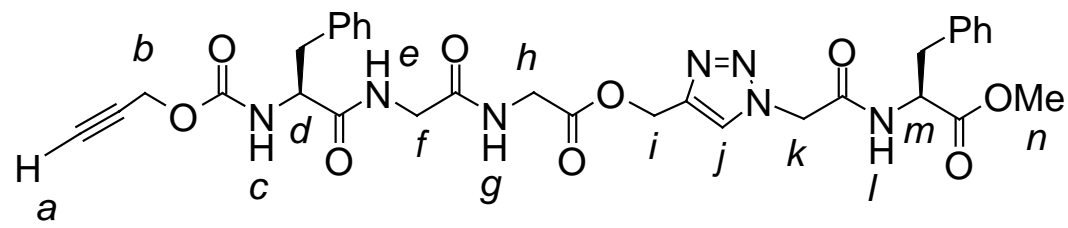

To a solution of TMS-alkyne 8 ( $69 \mathrm{mg}, 0.094 \mathrm{mmol})$ in acetone $(1 \mathrm{~mL})$ and water $(200 \mu \mathrm{L})$ were added $\mathrm{AgBF}_{4}$ (3.7 mg, 0.019 mmol, 0.2 equiv) The resulting solution was stirred at $\mathrm{rt}$ for $18 \mathrm{~h}$ then diluted with water $(10 \mathrm{~mL})$ and extracted with $\mathrm{CH}_{2} \mathrm{Cl}_{2}(4 \times 10 \mathrm{~mL})$. The combined organic layers were dried over $\mathrm{MgSO}_{4}$, filtered and concentrated in vacuo. The resulting yellow oil was purified using short path flash column chromatography on silica gel (eluent: $\mathrm{CH}_{2} \mathrm{Cl}_{2} / \mathrm{MeOH}$ gradient from 98/2 to 90/10) to give pure alkyne 9 as a colorless amorphous solid (56 $\mathrm{mg}, 90 \%$ ). $[\alpha]^{20}{ }_{\mathrm{D}}=+23.8\left(\mathrm{c} 1.0, \mathrm{CHCl}_{3}\right) ;{ }^{1} \mathrm{H} \mathrm{NMR}\left(400 \mathrm{MHz}, \mathrm{CDCl}_{3}, 298 \mathrm{~K}\right): \delta=2.44(\mathrm{t}, 1 \mathrm{H}, J=2.4 \mathrm{~Hz}$, $\left.\mathrm{H}_{\mathrm{a}}\right), 2.92-3.17\left(\mathrm{~m}, 4 \mathrm{H}, \mathrm{CH}_{2} \mathrm{Ph}\right), 3.69\left(\mathrm{~s}, 3 \mathrm{H}, \mathrm{H}_{\mathrm{n}}\right), 3.81\left(\mathrm{dd}, 1 \mathrm{H}, J=5.3 \mathrm{~Hz}\right.$ and $\left.16.9 \mathrm{~Hz}, \mathrm{H}_{\mathrm{fl}}\right)$, 3.89-4.08 (m, 3H, $\mathrm{H}_{\mathrm{f} 2}$ and $\left.\mathrm{H}_{\mathrm{h}}\right)$, 4.39-4.48 $\left(\mathrm{m}, 1 \mathrm{H}, \mathrm{H}_{\mathrm{d}}\right)$, $4.56\left(\mathrm{~s}, 2 \mathrm{H}, \mathrm{H}_{\mathrm{b}}\right), 4.79-4.87\left(\mathrm{~m}, 1 \mathrm{H}, \mathrm{H}_{\mathrm{m}}\right)$, $4.98\left(\mathrm{~d}, 1 \mathrm{H}, J=16.1 \mathrm{~Hz}, \mathrm{H}_{\mathrm{k} 1}\right), 5.04\left(\mathrm{~d}, 1 \mathrm{H}, J=16.1 \mathrm{~Hz}, \mathrm{H}_{\mathrm{k} 2}\right), 5.20\left(\mathrm{~s}, 2 \mathrm{H}, \mathrm{H}_{\mathrm{i}}\right), 5.99(\mathrm{br} \mathrm{d}, 1 \mathrm{H}, J=$ $\left.7.3 \mathrm{~Hz}, \mathrm{H}_{\mathrm{c}}\right), 7.01-7.33\left(\mathrm{~m}, 13 \mathrm{H}, \mathrm{H}_{\mathrm{Ar}}, \mathrm{H}_{\mathrm{g}}, \mathrm{H}_{\mathrm{e}}\right.$ and $\left.\mathrm{H}_{\mathrm{l}}\right), 7.75\left(\mathrm{~s}, 1 \mathrm{H}, \mathrm{H}_{\mathrm{j}}\right) ;{ }^{13} \mathrm{C}$ NMR $(100 \mathrm{MHz}$, $\left.\mathrm{CDCl}_{3}, 298 \mathrm{~K}\right): \delta=37.5\left(\mathrm{CH}_{2}\right), 37.9\left(\mathrm{CH}_{2}\right), 41.2\left(\mathrm{CH}_{2}\right), 42.6\left(\mathrm{CH}_{2}\right), 52.2\left(\mathrm{CH}_{2}\right), 52.6(\mathrm{CH}), 52.9$ $\left(\mathrm{CH}_{2}\right), 53.8(\mathrm{CH}), 56.6\left(\mathrm{CH}_{3}\right), 58.2\left(\mathrm{CH}_{2}\right), 75.1(\mathrm{CH}), 77.9(\mathrm{C}), 125.7(\mathrm{CH}), 127.1(\mathrm{CH}), 127.2$ $(\mathrm{CH}), 128.67(\mathrm{CH}), 128.72(\mathrm{CH}), 129.16(\mathrm{CH}), 129.24(\mathrm{CH}), 135.7(\mathrm{C}), 136.3(\mathrm{C}), 142.5(\mathrm{C})$, 155.8 (C), 165.2 (C), 169.7 (C), 169.9 (C), 171.8 (C), 171.2 (C); LRFAB-MS (3-NOBA matrix): $\mathrm{m} / \mathrm{z}=662[\mathrm{MH}]^{+}$.

\section{General procedure for the $\mathrm{Cu}(\mathrm{I})$ then $\operatorname{Ag}(\mathrm{I})$ one-pot succesive formation of two triazole linkages:}

To a solution of dialkyne 5 (47 mg, $0.1 \mathrm{mmol})$ and azide-modified dipeptide $7 \mathbf{a}$ (26 mg, 0.1 mmol, 1 equiv) in $t$ - $\mathrm{BuOH}(4 \mathrm{~mL})$ and water $(200 \mu \mathrm{L})$ were added $\mathrm{CuSO}_{4}(0.1 \mathrm{M}$ in water, 100 $\mu \mathrm{L}, 0.1$ equiv) and sodium ascorbate $(0.1 \mathrm{M}$ in water, $200 \mu \mathrm{L}, 0.2$ equiv). The resulting solution was stirred at $35^{\circ} \mathrm{C}$ for $18 \mathrm{~h}$ and then the azide-modified dipeptide methyl ester $7 \mathbf{b}, 7 \mathbf{c}$, or $7 \mathbf{d}(1.2$ mmol, 1.2 equiv) and $\mathrm{AgBF}_{4}(38 \mathrm{mg}, 0.2 \mathrm{mmol}, 0.2$ equiv) were added. The dark suspension was stirred at $35{ }^{\circ} \mathrm{C}$ for $24 \mathrm{~h}$ following which a new batch of $\mathrm{CuSO}_{4}(0.1 \mathrm{M}$ in water, $100 \mu \mathrm{L}, 0.1$ equiv) and sodium ascorbate $(0.1 \mathrm{M}$ in water, $200 \mu \mathrm{L}, 0.2$ equiv) were added. The resulting suspension was stirred at $35{ }^{\circ} \mathrm{C}$ for $18 \mathrm{~h}$ then diluted with water $(10 \mathrm{~mL})$ and extracted with $\mathrm{CH}_{2} \mathrm{Cl}_{2}(4 \times 10 \mathrm{~mL})$. The combined organic layers were dried over $\mathrm{MgSO}_{4}$, filtered and concentrated in vacuo. Resulting yellow oil was purified using short path flash column chromatography on silica gel (eluent: $\mathrm{CH}_{2} \mathrm{Cl}_{2} / \mathrm{MeOH}$ gradient from 98:2 to 88:12) to give pure triazoles 10a-c as colorless amorphous solids. 
Bis-triazole pseudononapeptide (10a)

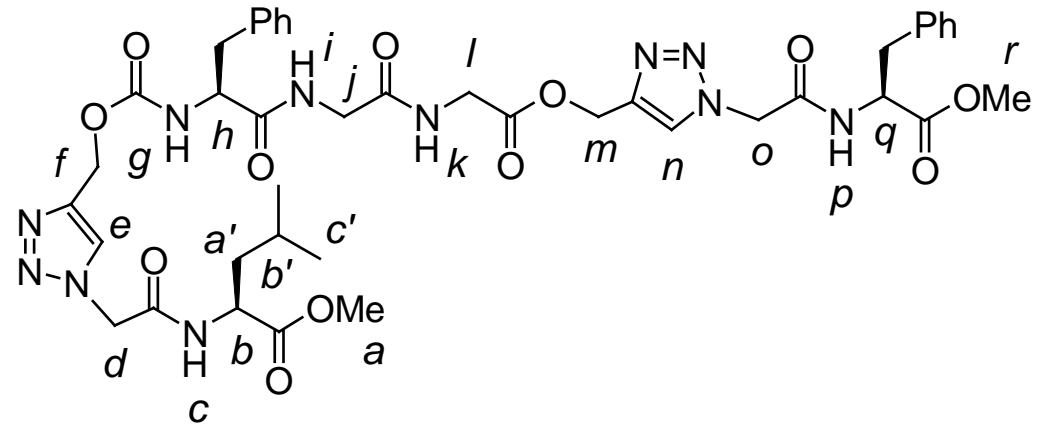

Obtained from 5, 7a and 7b following the general procedure. Colorless oil $(83 \mathrm{mg}, 93 \%)$. $[\alpha]^{20}{ }_{\mathrm{D}}$ $=+32.9\left(\mathrm{c} 1.0, \mathrm{CHCl}_{3}\right) ;{ }^{1} \mathrm{H} \mathrm{NMR}\left(400 \mathrm{MHz}, \mathrm{CDCl}_{3} / \mathrm{CD}_{3} \mathrm{OD} 8: 2,298 \mathrm{~K}\right): \delta=0.87(\mathrm{~d}, 3 \mathrm{H}, J=5.4$ $\left.\mathrm{Hz}, \mathrm{H}_{\mathrm{c}^{\prime} 1}\right), 0.92\left(\mathrm{~d}, 3 \mathrm{H}, J=5.4 \mathrm{~Hz}, \mathrm{H}_{\mathrm{c}^{\prime} 2}\right), 1.46-1.68\left(\mathrm{~m}, 3 \mathrm{H}, \mathrm{H}_{\mathrm{a}}\right.$ and $\left.\mathrm{H}_{\mathrm{b}^{\prime}}\right), 2.86-3.19(\mathrm{~m}, 4 \mathrm{H}$, $\left.\mathrm{CH}_{2} \mathrm{Ph}\right), 3.67\left(\mathrm{~s}, 6 \mathrm{H}, \mathrm{H}_{\mathrm{a}}\right.$ and $\left.\mathrm{H}_{\mathrm{r}}\right), 3.78\left(\mathrm{~d}, 1 \mathrm{H}, J=16.0 \mathrm{~Hz}, \mathrm{H}_{\mathrm{j} 1}\right), 3.83-4.02\left(\mathrm{~m}, 3 \mathrm{H}, \mathrm{H}_{\mathrm{j} 2}\right.$ and $\left.\mathrm{H}_{\mathrm{i}}\right)$, 4.24-4.35 (m, $\left.1 \mathrm{H}, \mathrm{H}_{\mathrm{h}}\right), 4.43-4.51\left(\mathrm{~m}, 1 \mathrm{H}, \mathrm{H}_{\mathrm{b}}\right)$, 4.68-4.77 (m, 1H, $\left.\mathrm{H}_{\mathrm{q}}\right), 4.98-5.27\left(\mathrm{~m}, 8 \mathrm{H}, \mathrm{H}_{\mathrm{d}}, \mathrm{H}_{\mathrm{f}}\right.$, $\mathrm{H}_{\mathrm{m}}$ and $\left.\mathrm{H}_{\mathrm{o}}\right)$, 7.08-7.32 (m, 10H, $\left.\mathrm{H}_{\mathrm{Ar}}\right), 7.80\left(\mathrm{~s}, 2 \mathrm{H}, \mathrm{H}_{\mathrm{e}}\right.$ and $\left.\mathrm{H}_{\mathrm{n}}\right) ;{ }^{13} \mathrm{C} \mathrm{NMR}(100 \mathrm{MHz}$, $\left.\mathrm{CDCl}_{3} / \mathrm{CD}_{3} \mathrm{OD} 8: 2,298 \mathrm{~K}\right): \delta=20.9\left(\mathrm{CH}_{3}\right), 22.2\left(\mathrm{CH}_{3}\right), 24.3(\mathrm{CH}), 29.2\left(\mathrm{CH}_{2}\right), 37.1\left(\mathrm{CH}_{2}\right), 40.1$ $\left(\mathrm{CH}_{2}\right), 40.6\left(\mathrm{CH}_{2}\right), 42.0\left(\mathrm{CH}_{2}\right), 50.8(\mathrm{CH}), 51.8\left(\mathrm{CH}_{2}\right), 51.9\left(\mathrm{CH}_{2}\right), 52.2\left(\mathrm{CH}_{3}\right), 52.3\left(\mathrm{CH}_{3}\right), 53.6$ $(\mathrm{CH}), 56.2\left(\mathrm{CH}_{2}\right), 56.5(\mathrm{CH}), 57.5\left(\mathrm{CH}_{2}\right), 57.6\left(\mathrm{CH}_{2}\right), 125.5(\mathrm{CH}), 125.6(\mathrm{CH}), 126.4(\mathrm{CH})$, $126.6(\mathrm{CH}), 128.1(\mathrm{CH}), 128.2(\mathrm{CH}), 128.7(\mathrm{CH}), 135.7(\mathrm{C}), 136.4(\mathrm{C}), 142.0(\mathrm{C}), 142.7(\mathrm{C})$, 156.3 (C), 165.4 (C), 165.7 (C), 169.2 (C), 170.1 (C), 171.5 (C), 172.6 (C), 172.9 (C); LRFABMS (3-NOBA matrix): $m / z=890[\mathrm{MH}]^{+}$; HRFAB-MS (3-NOBA matrix): $\mathrm{m} / z=890.37928$ (calcd. for $\mathrm{C}_{41} \mathrm{H}_{52} \mathrm{~N}_{11} \mathrm{O}_{12}, 890.37969$ ).

\section{Bis-triazole pseudononapeptide (10b)}

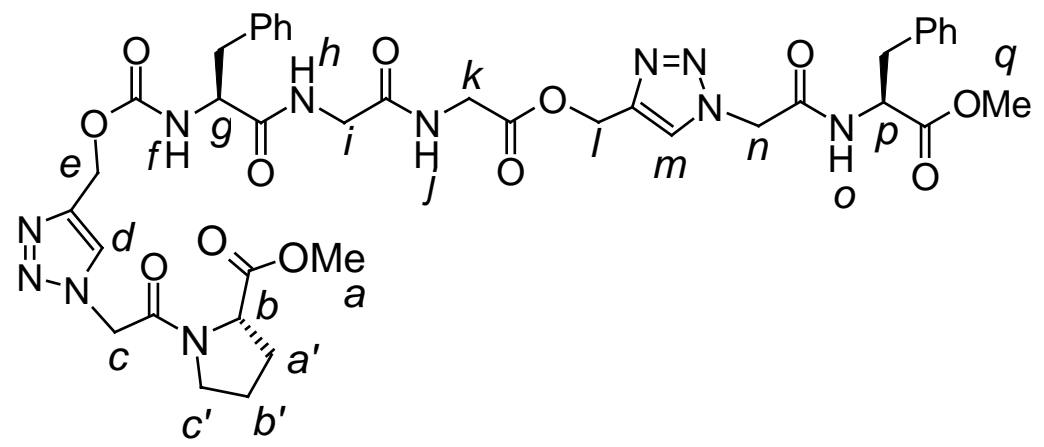

Obtained from 5, 7a and 7c following the general procedure. Colorless oil $(79 \mathrm{mg}, 91 \%)$. $[\alpha]^{20}{ }_{\mathrm{D}}=$ -8.4 (c 1.0, $\left.\mathrm{CHCl}_{3}\right) ;{ }^{1} \mathrm{H} \mathrm{NMR}\left(400 \mathrm{MHz}, \mathrm{CDCl}_{3} / \mathrm{CD}_{3} \mathrm{OD}\right.$ 95:5, $298 \mathrm{~K}$ ): 82:18 mixture of two rotamers, major $(\mathrm{M})$ and minor $(\mathrm{m}) \quad \delta=1.79-2.31\left(\mathrm{~m}, 4 \mathrm{H}, \mathrm{H}_{\mathrm{a}}\right.$, and $\left.\mathrm{H}_{\mathrm{b}},\right), 2.85-3.17(\mathrm{~m}, 4 \mathrm{H}$, $\left.\mathrm{CH}_{2} \mathrm{Ph}\right), 3.45-3.96\left(\mathrm{~m}, 6 \mathrm{H}, \mathrm{H}_{\mathrm{i}}, \mathrm{H}_{\mathrm{k}}\right.$ and $\left.\mathrm{H}_{\mathrm{c}^{\prime}}\right), 3.66\left(\mathrm{~s}, 5.46 \mathrm{H}, \mathrm{H}_{\mathrm{q}}\right.$ and $\left.\mathrm{H}_{\mathrm{a}(\mathrm{M})}\right), 3.77\left(\mathrm{~s}, 0.54 \mathrm{H}, \mathrm{H}_{\mathrm{c}(\mathrm{m})}\right)$, 4.23-4.32 (m, $\left.1 \mathrm{H}, \mathrm{H}_{\mathrm{g}}\right), 4.46\left(\mathrm{dd}, 0.82 \mathrm{H}, J=3.8 \mathrm{~Hz}, 8.5 \mathrm{~Hz}, \mathrm{H}_{\mathrm{b}(\mathrm{M})}\right), 4.63(\mathrm{dd}, 0.18 \mathrm{H}, J=5.2 \mathrm{~Hz}$, $\left.5.6 \mathrm{~Hz}, \mathrm{H}_{\mathrm{b}(\mathrm{m})}\right), 4.70-4.78\left(\mathrm{~m}, 1 \mathrm{H}, \mathrm{H}_{\mathrm{p}}\right), 4.89-5.29\left(\mathrm{~m}, 8 \mathrm{H}, \mathrm{H}_{\mathrm{c}}, \mathrm{H}_{\mathrm{e}}, \mathrm{H}_{1}\right.$ and $\left.\mathrm{H}_{\mathrm{n}}\right), 6.16(\mathrm{br} \mathrm{d}, 0.18 \mathrm{H}, J=$ $\left.6.2 \mathrm{~Hz}, \mathrm{H}_{\mathrm{f}(\mathrm{m})}\right), 6.23$ (br d, 0.82H, $\left.J=6.2 \mathrm{~Hz}, \mathrm{H}_{\mathrm{f}(\mathrm{M})}\right), 7.01-7.28\left(\mathrm{~m}, 10 \mathrm{H}, \mathrm{H}_{\mathrm{Ar}}\right), 7.39-7.89(\mathrm{~m}, 5 \mathrm{H}$, $\left.\mathrm{H}_{\mathrm{d}}, \mathrm{H}_{\mathrm{h}}, \mathrm{H}_{\mathrm{j}}, \mathrm{H}_{\mathrm{m}}\right) ;{ }^{13} \mathrm{C} \mathrm{NMR}\left(100 \mathrm{MHz}, \mathrm{CDCl}_{3} / \mathrm{CD}_{3} \mathrm{OD}\right.$ 95:5, $\left.298 \mathrm{~K}\right)$ : mixture of 2 rotamers, major 
(M) and minor $(\mathrm{m}) \quad \delta=22.3\left(\mathrm{~m}, \mathrm{CH}_{2}\right), 24.7\left(\mathrm{M}, \mathrm{CH}_{2}\right), 28.9\left(\mathrm{M}, \mathrm{CH}_{2}\right), 29.7\left(\mathrm{~m}, \mathrm{CH}_{2}\right), 31.2$ $\left(\mathrm{CH}_{2}\right)$, $37.5\left(\mathrm{CH}_{2}\right), 41.1\left(\mathrm{CH}_{2}\right), 41.2\left(\mathrm{CH}_{2}\right), 42.6\left(\mathrm{CH}_{2}\right), 42.7\left(\mathrm{CH}_{2}\right), 46.6\left(\mathrm{M}, \mathrm{CH}_{2}\right), 47.2(\mathrm{~m}$, $\left.\mathrm{CH}_{2}\right), 51.4\left(\mathrm{CH}_{2}\right), 52.1\left(\mathrm{CH}_{2}\right), 52.47(\mathrm{CH}), 52.54(\mathrm{CH}), 53.1\left(\mathrm{~m}, \mathrm{CH}_{3}\right), 53.6\left(\mathrm{CH}_{3}\right), 53.7(\mathrm{M}$, $\left.\mathrm{CH}_{3}\right), 58.05\left(\mathrm{~m}, \mathrm{CH}_{2}\right), 58.13\left(\mathrm{M}, \mathrm{CH}_{2}\right), 59.2(\mathrm{~m}, \mathrm{CH}), 59.3(\mathrm{M}, \mathrm{CH}), 126.95(\mathrm{M}, \mathrm{CH}), 127.04(\mathrm{~m}$, $\mathrm{CH}), 127.1(\mathrm{CH}), 128.5(\mathrm{CH}), 128.59(\mathrm{CH}), 128.62(\mathrm{CH}), 128.7(\mathrm{CH}), 129.1(\mathrm{CH}), 129.2(\mathrm{CH})$, 135.7 (C), 136.5 (C), 142.5 (C), 142.8 (M, C), 142.9 (m, C), 156.4 (C), 164.2 (M, C), 164.6 (m, C), 165.1 (C), 165.2 (C), 169.7 (C), 170.1 (M, C), 170.2 (m, C), 171.71 (C), 171.73 (C), 171.8 (C), 172.3 (C); LRFAB-MS (3-NOBA matrix): $m / z=874[M H]^{+}$; HRFAB-MS (3-NOBA matrix): $m / z=874.34811$ (calcd. for $\mathrm{C}_{40} \mathrm{H}_{48} \mathrm{~N}_{11} \mathrm{O}_{12}, 874.34839$ ).

\section{Bis-triazole pseudononapeptide (10c)}

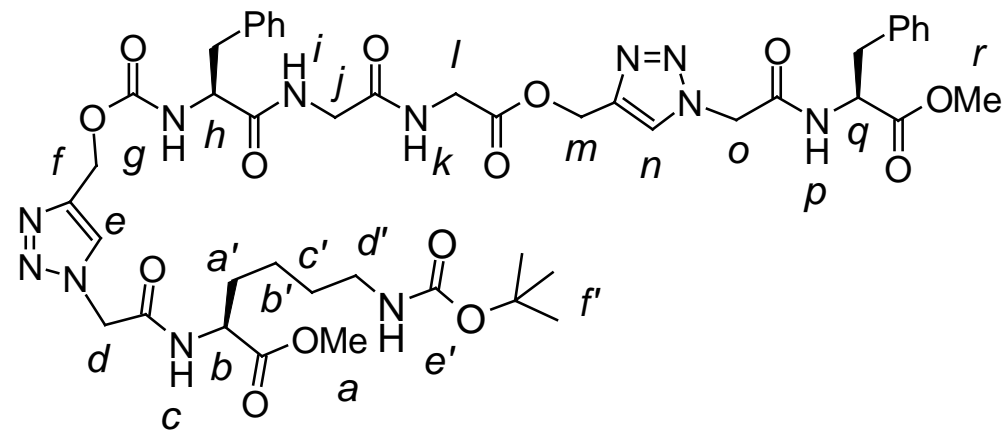

Obtained from 5, 7a and 7e following the general procedure. Colorless oil $(88 \mathrm{mg}, 88 \%)$. $[\alpha]^{20}{ }_{\mathrm{D}}=$ $+15.5\left(\mathrm{c} 1.0, \mathrm{CHCl}_{3}\right) ;{ }^{1} \mathrm{H}$ NMR (500 MHz, $\left.\mathrm{CDCl}_{3} / \mathrm{CD}_{3} \mathrm{OD} 9: 1,298 \mathrm{~K}\right): \delta=1.22-1.32(\mathrm{~m}, 2 \mathrm{H}$, $\left.\mathrm{H}_{\mathrm{b}^{\prime}}\right), 1.37\left(\mathrm{~s}, 9 \mathrm{H}, \mathrm{H}_{\mathrm{f}}\right), 1.37-1.45\left(\mathrm{~m}, 2 \mathrm{H}, \mathrm{H}_{\mathrm{c}^{\prime}}\right), 1.58-1.69\left(\mathrm{~m}, 1 \mathrm{H}, \mathrm{H}_{\mathrm{a}^{\prime} 1}\right), 1.71-1.82\left(\mathrm{~m}, 1 \mathrm{H}, \mathrm{H}_{\mathrm{a}^{\prime} 2}\right)$, 2.81-3.12 $\left(\mathrm{m}, 6 \mathrm{H}, \mathrm{H}_{\mathrm{d}^{\prime}}\right.$ and $\left.\mathrm{CH}_{2} \mathrm{Ph}\right), 3.63$ and $3.64\left(2 \mathrm{~s}, 2 \times 3 \mathrm{H}, \mathrm{H}_{\mathrm{a}}\right.$ and $\left.\mathrm{H}_{\mathrm{r}}\right), 3.71(\mathrm{~d}, 1 \mathrm{H}, J=16.8$ $\left.\mathrm{Hz}, \mathrm{H}_{\mathrm{j} 1}\right), 3.85\left(\mathrm{~d}, 1 \mathrm{H}, J=16.8 \mathrm{~Hz}, \mathrm{H}_{\mathrm{j} 2}\right), 3.88\left(\mathrm{~d}, 1 \mathrm{H}, J=17.1 \mathrm{~Hz}, \mathrm{H}_{11}\right), 3.92(\mathrm{~d}, 1 \mathrm{H}, J=17.1 \mathrm{~Hz}$, $\left.\mathrm{H}_{12}\right)$, 4.21-4.27 (m, $\left.1 \mathrm{H}, \mathrm{H}_{\mathrm{h}}\right)$, 4.35-4.41 (m, $\left.1 \mathrm{H}, \mathrm{H}_{\mathrm{b}}\right)$, 4.66-4.72 (m, 1H, $\left.\mathrm{H}_{\mathrm{q}}\right)$, 4.96-5.12 (m, 6H, $\mathrm{H}_{\mathrm{f}}$, $\mathrm{H}_{\mathrm{m}}$ and $\left.\mathrm{H}_{\mathrm{o}}\right), 5.18\left(\mathrm{~s}, 2 \mathrm{H}, \mathrm{H}_{\mathrm{d}}\right), 7.03-7.22\left(\mathrm{~m}, 10 \mathrm{H}, \mathrm{H}_{\mathrm{Ar}}\right), 7.74$ and $7.76\left(2 \mathrm{~s}, 2 \mathrm{x} 1 \mathrm{H}, \mathrm{H}_{\mathrm{e}}\right.$ and $\left.\mathrm{H}_{\mathrm{n}}\right) ;{ }^{13} \mathrm{C}$ NMR (126 MHz, CDCl $3 / \mathrm{CD}_{3} \mathrm{OD}$ 9:1, $\left.298 \mathrm{~K}\right): \delta=22.6\left(\mathrm{CH}_{2}\right), 28.5\left(\mathrm{CH}_{3}\right), 29.6\left(\mathrm{CH}_{2}\right), 31.4$ $\left(\mathrm{CH}_{2}\right), 37.6\left(\mathrm{CH}_{2}\right), 37.7\left(\mathrm{CH}_{2}\right), 40.1\left(\mathrm{CH}_{2}\right), 41.4\left(\mathrm{CH}_{2}\right), 43.1\left(\mathrm{CH}_{2}\right), 52.3\left(\mathrm{CH}_{2}\right), 52.4\left(\mathrm{CH}_{2}\right)$, $52.46\left(\mathrm{CH}_{3}\right), 52.54\left(\mathrm{CH}_{3}\right), 52.6(\mathrm{CH}), 53.8(\mathrm{CH}), 57.0(\mathrm{CH}), 58.4\left(\mathrm{CH}_{2}\right), 58.5\left(\mathrm{CH}_{2}\right), 77.4(\mathrm{C})$, $125.59(\mathrm{CH}), 125.64(\mathrm{CH}), 127.1(\mathrm{CH}), 127.3(\mathrm{CH}), 128.7(\mathrm{CH}), 128.8(\mathrm{CH}), 129.29(\mathrm{CH})$, $129.31(\mathrm{CH}), 135.8(\mathrm{C}), 136.8(\mathrm{C}), 142.8(\mathrm{C}), 142.9(\mathrm{C}), 156.4(\mathrm{C}), 156.5(\mathrm{C}), 165.2(\mathrm{C}), 165.6$ (C), 169.6 (C), 169.8 (C), 171.9 (C), 172.1 (C), 172.4 (C); LRFAB-MS (3-NOBA matrix): $\mathrm{m} / \mathrm{z}=$ $1005[M H]+$; HRFAB-MS (3-NOBA matrix): $\mathrm{m} / z=1005.44287$ (calcd. for C46H61N12O14, 1005.44302).

\section{References}

(1) Aucagne, V.; Hänni, K. D.; Leigh, D. A.; Lusby, P. J.; Walker, D. B. J. Am. Chem. Soc. 2006, $128,2186-2187$

.(2) (a) Homer, J.; Perry, M. C. J. Chem. Soc. Chem. Commun. 1994, 373-374 (b) Homer, J.; Perry, M. C. J. Chem. Soc. Perkin Trans. 2 1995, 533-536. 


\section{NMR and HPLC Characterization}

${ }^{1} \mathrm{H} \mathrm{NMR}$ of 2 in $\mathrm{CDCl}_{3}, 400 \mathrm{MHz}, \mathrm{rt}$

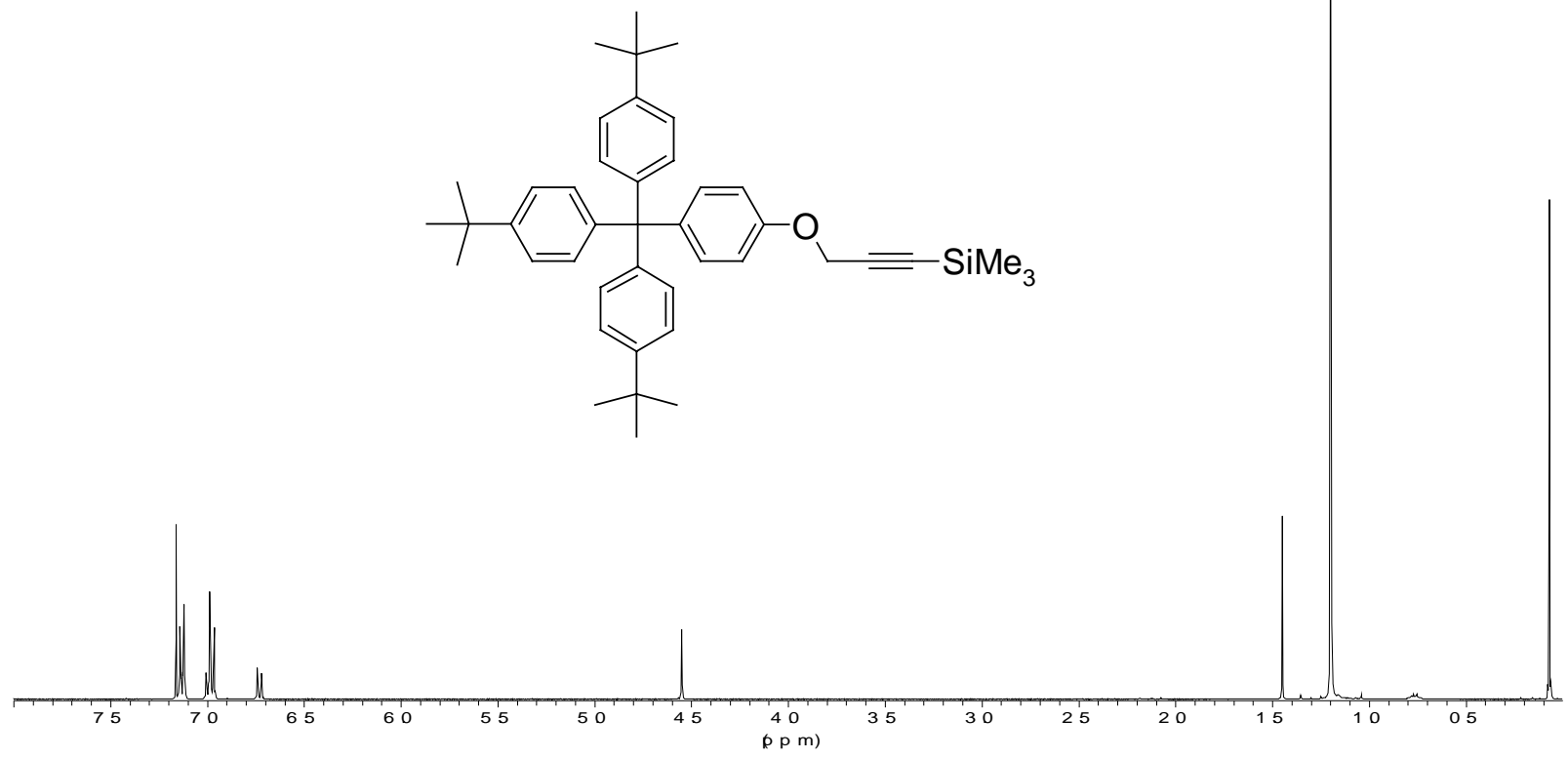

${ }^{13} \mathrm{C}$ pendant $\mathrm{NMR}$ of 2 in $\mathrm{CDCl}_{3}, 100 \mathrm{MHz}, \mathrm{rt}$

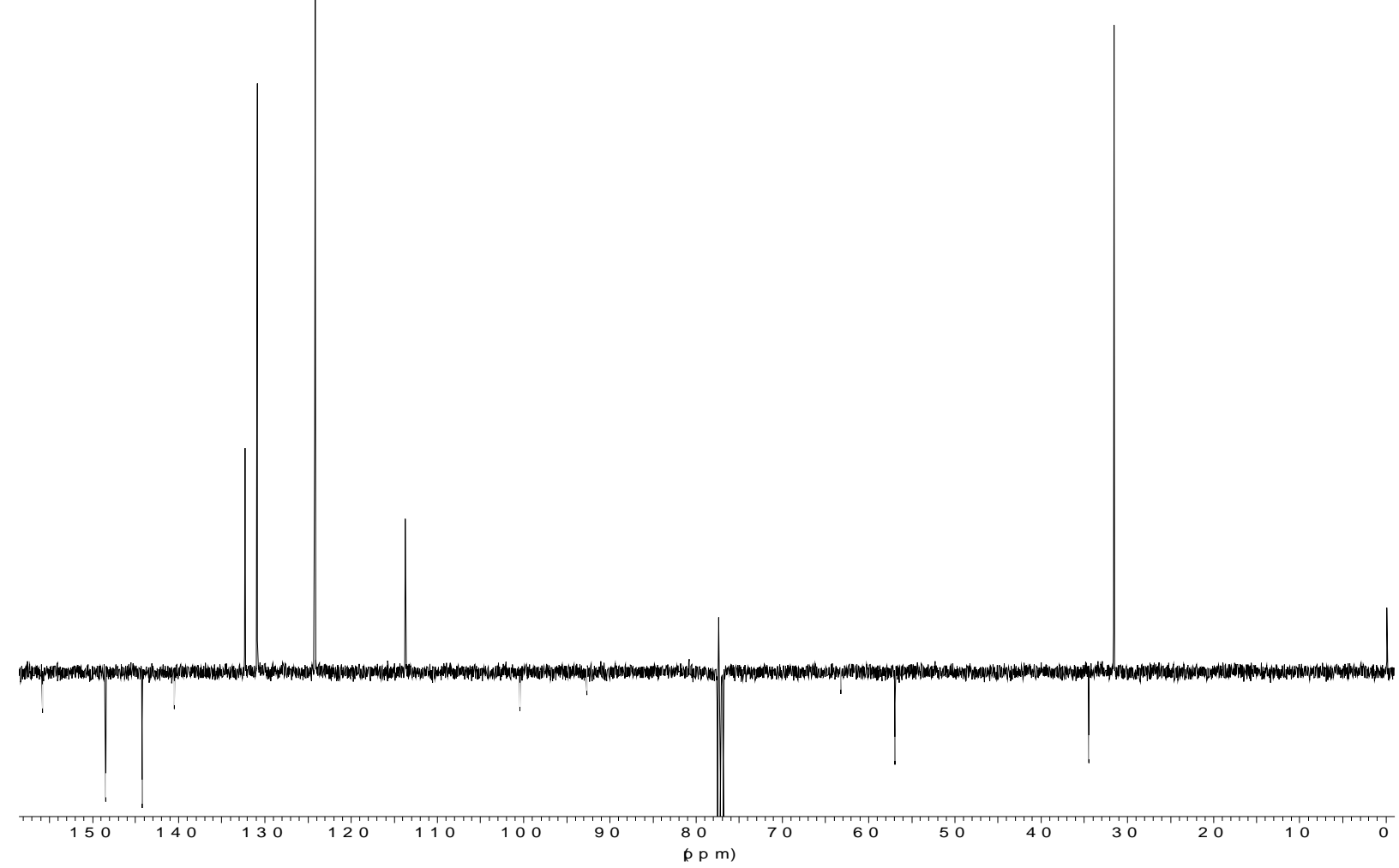


${ }^{1} \mathrm{H}$ NMR of 5 in $\mathrm{CDCl}_{3}, 400 \mathrm{MHz}$, rt

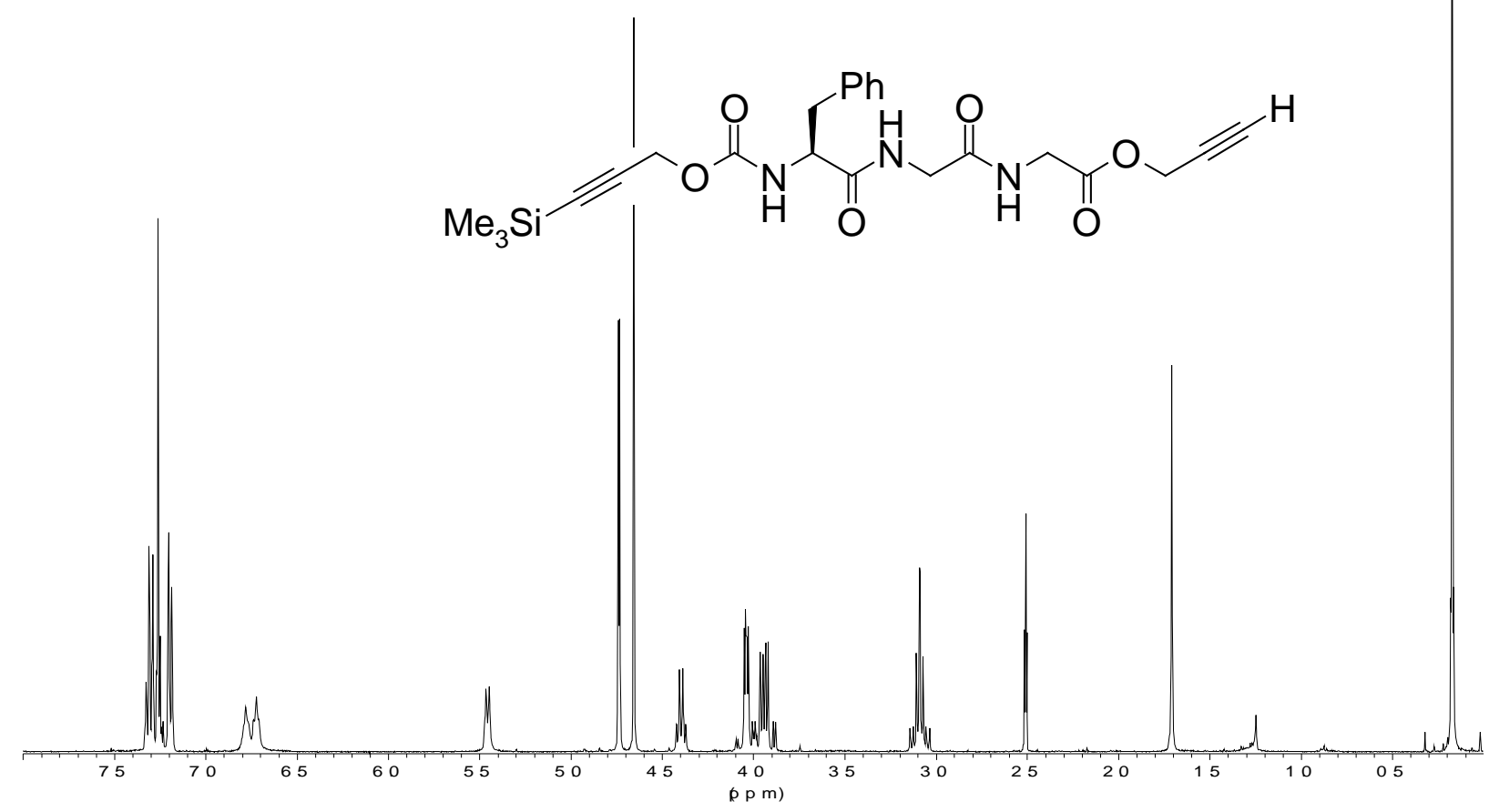

${ }^{13} \mathrm{C}$ pendant NMR of 5 in $\mathrm{CDCl}_{3}, 100 \mathrm{MHz}, \mathrm{rt}$

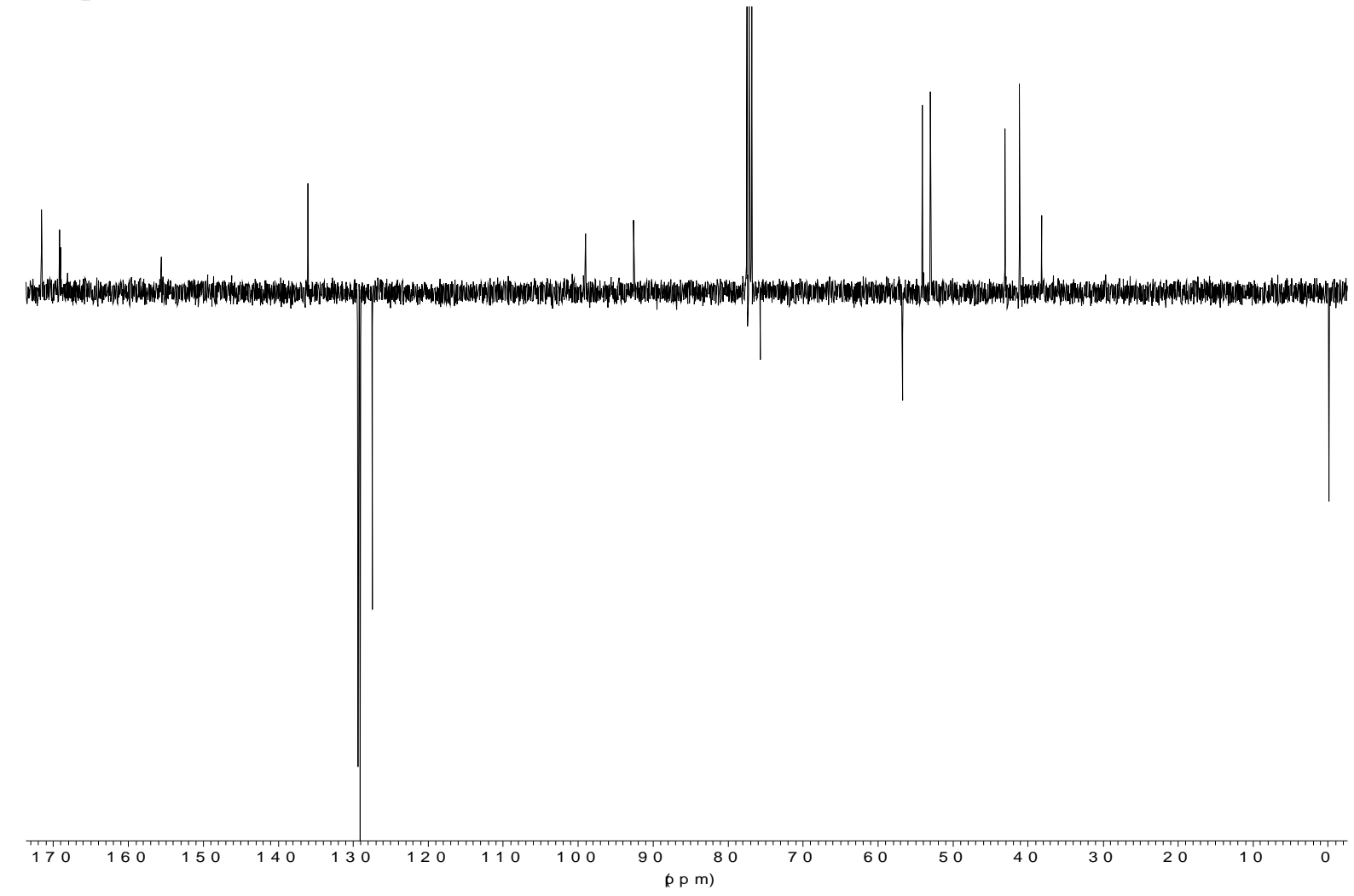


HPLC chromatogram of $\mathbf{5}$, gradient B
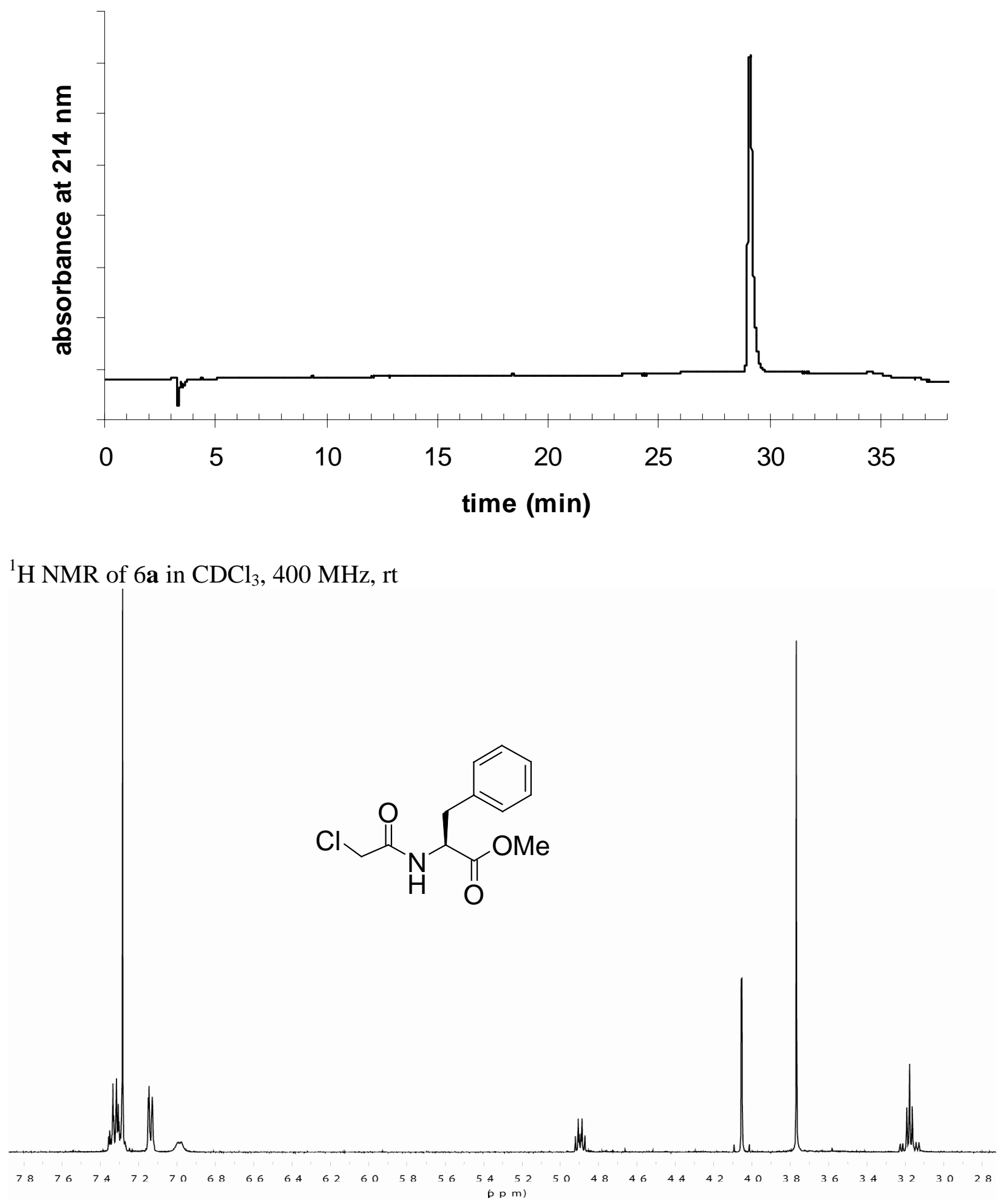


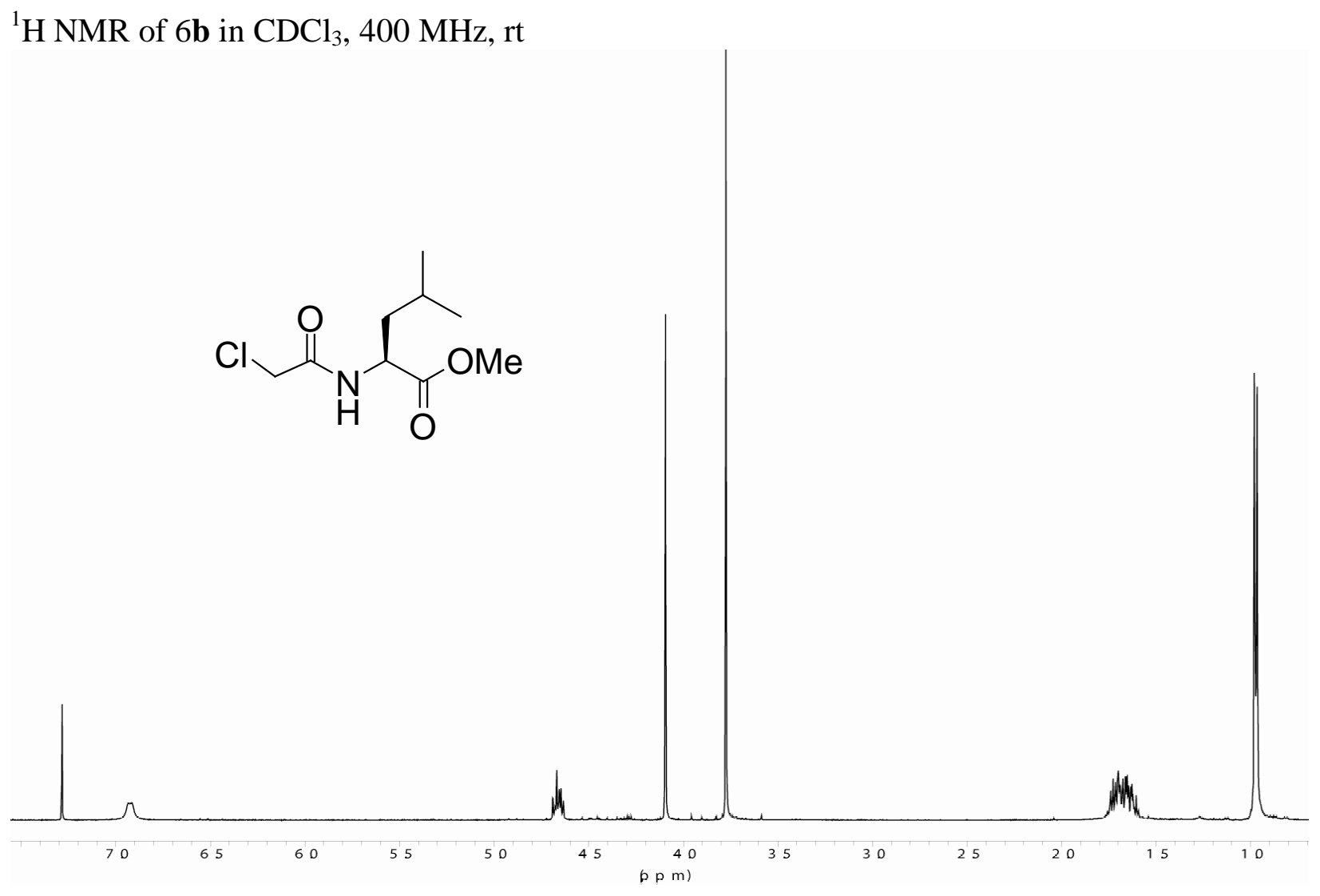

${ }^{1} \mathrm{H} \mathrm{NMR}$ of $6 \mathbf{c}$ in $\mathrm{CDCl}_{3}, 400 \mathrm{MHz}, \mathrm{rt}$

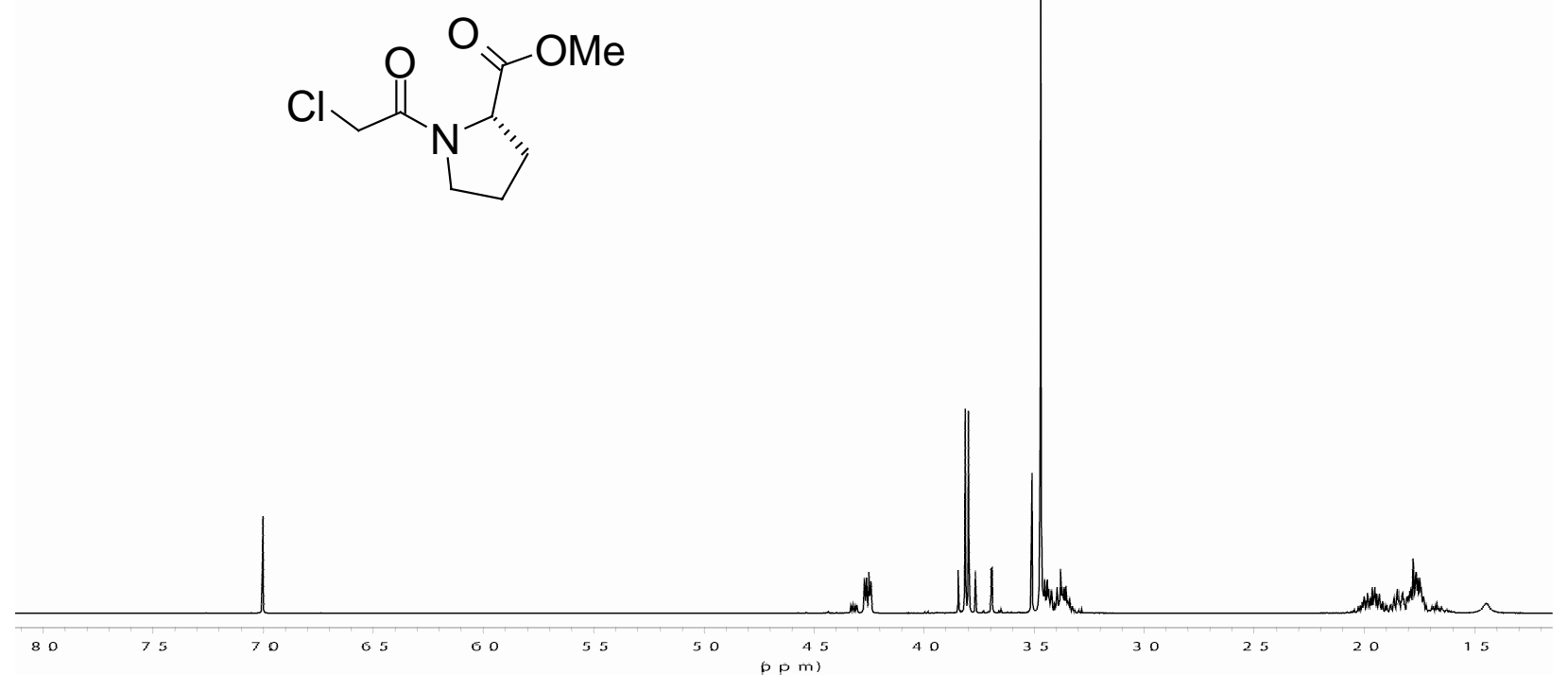


${ }^{1} \mathrm{H}$ NMR of $6 \mathbf{d}$ in $\mathrm{CDCl}_{3}, 400 \mathrm{MHz}, \mathrm{rt}$

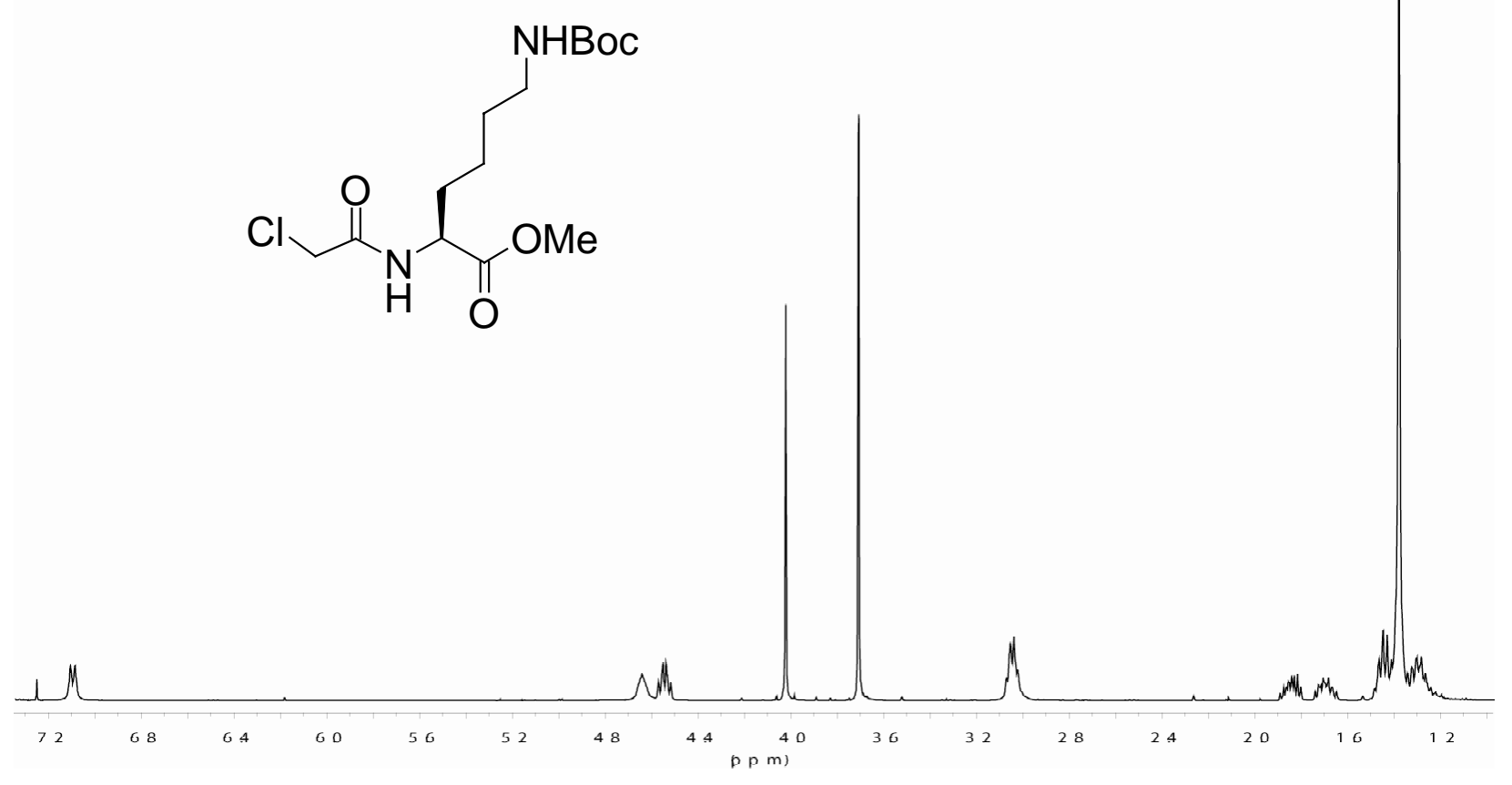

${ }^{1} \mathrm{H}$ NMR of 7a in $\mathrm{CDCl}_{3}, 400 \mathrm{MHz}$, rt

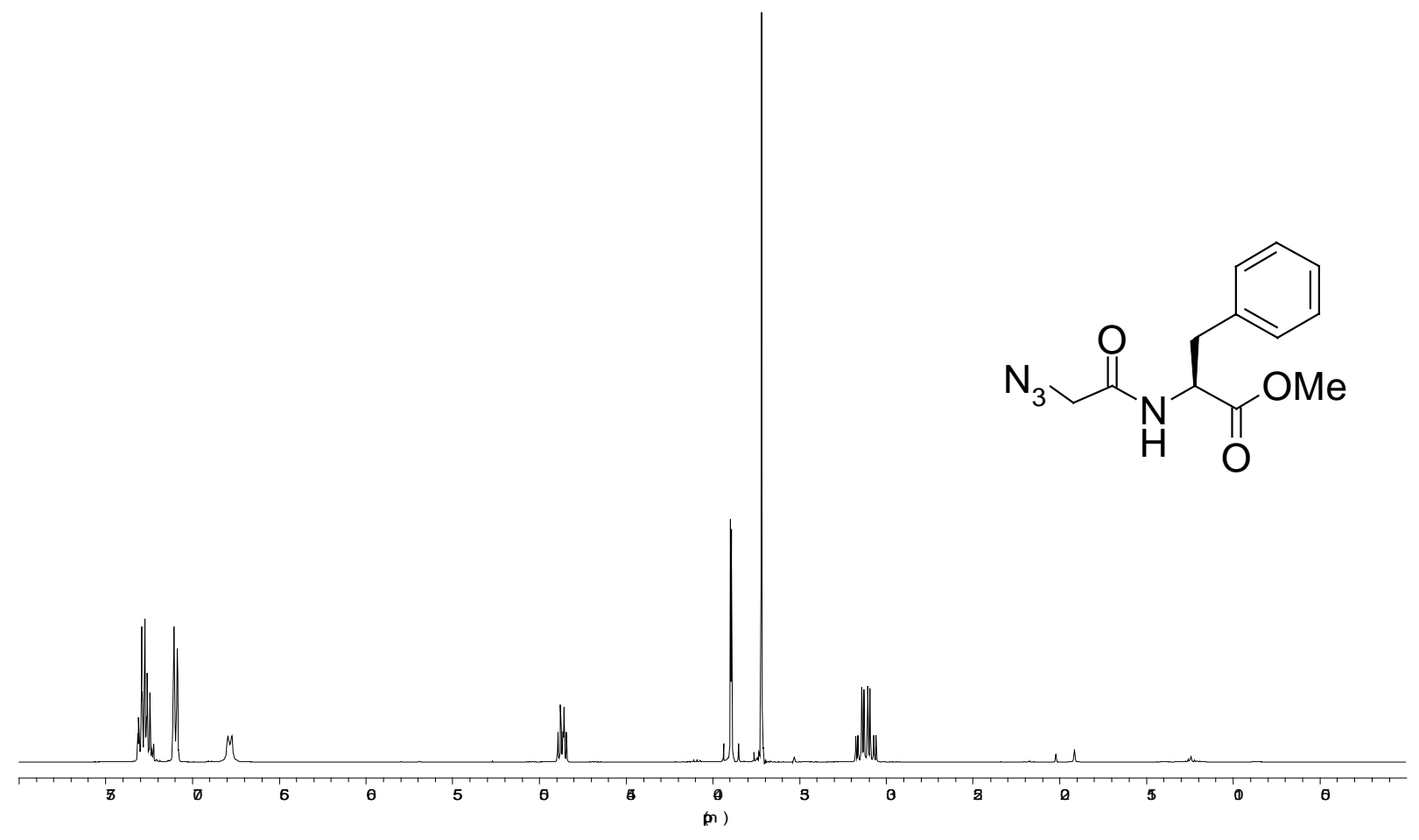


${ }^{13} \mathrm{C}$ NMR of $7 \mathbf{a}$ in $\mathrm{CDCl}_{3}, 62.5 \mathrm{MHz}, \mathrm{rt}$

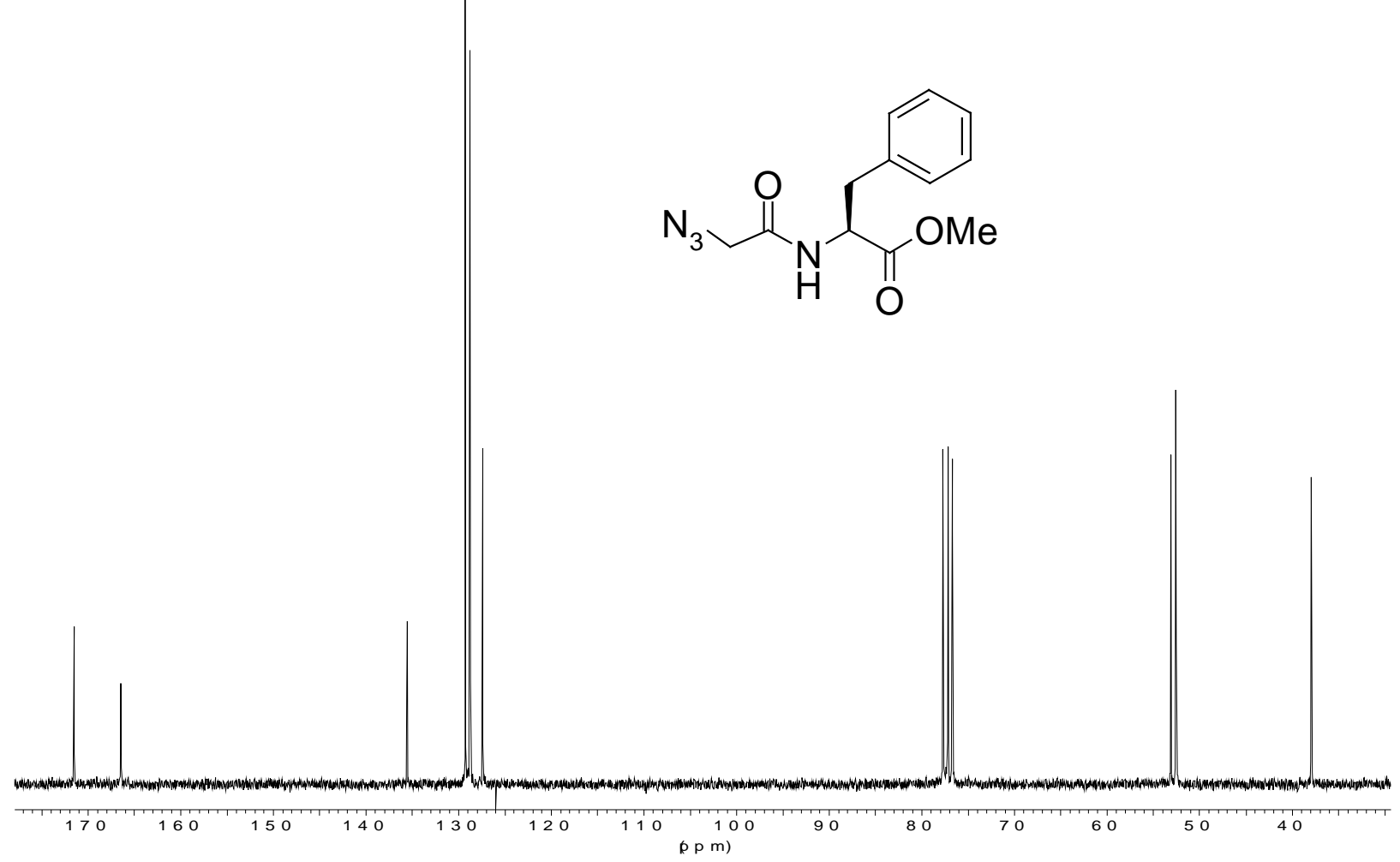

${ }^{13} \mathrm{C}$ pendant $\mathrm{NMR}$ of $\mathbf{7 a}$ in $\mathrm{CDCl}_{3}, 100 \mathrm{MHz}, \mathrm{rt}$

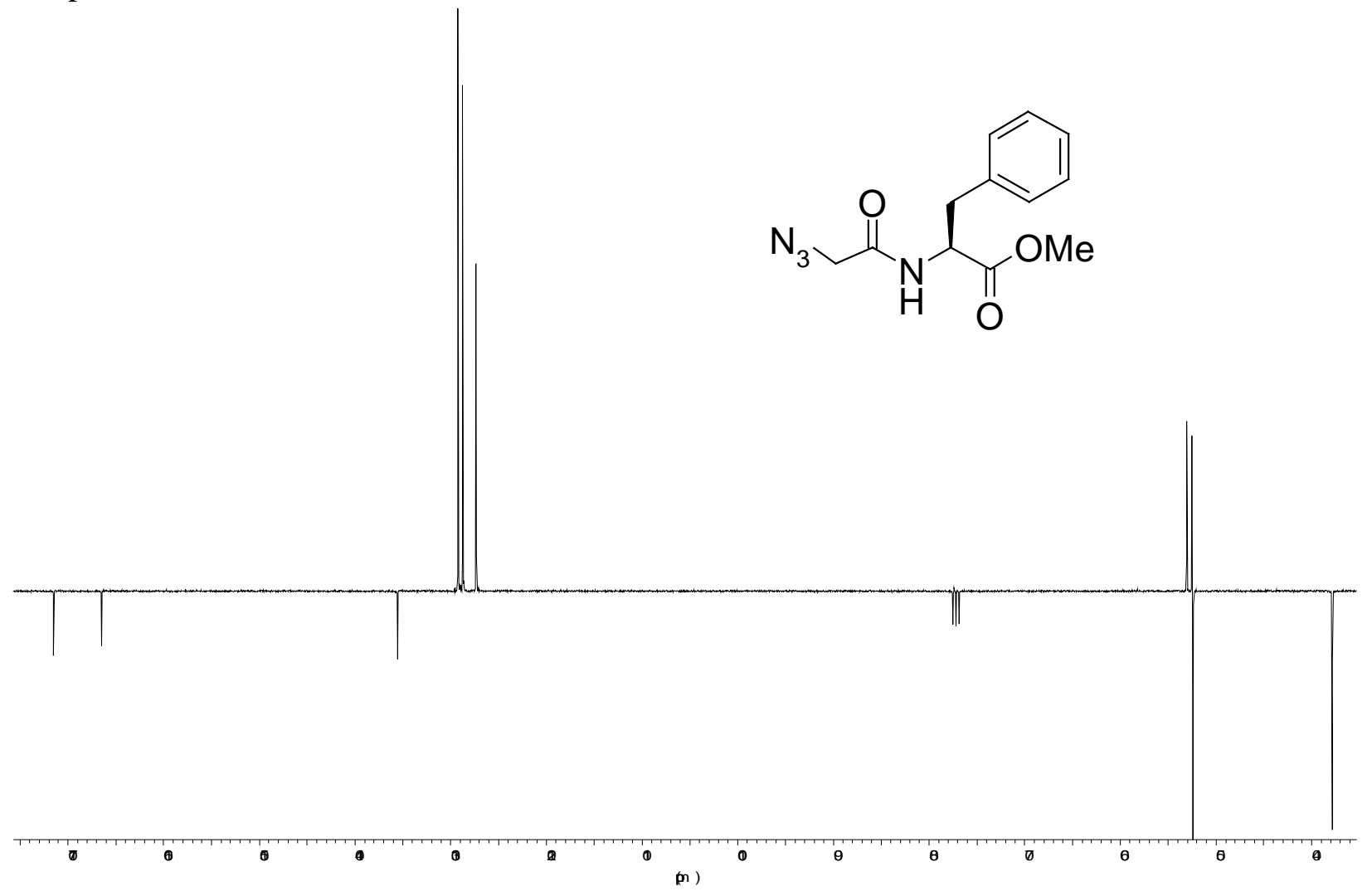




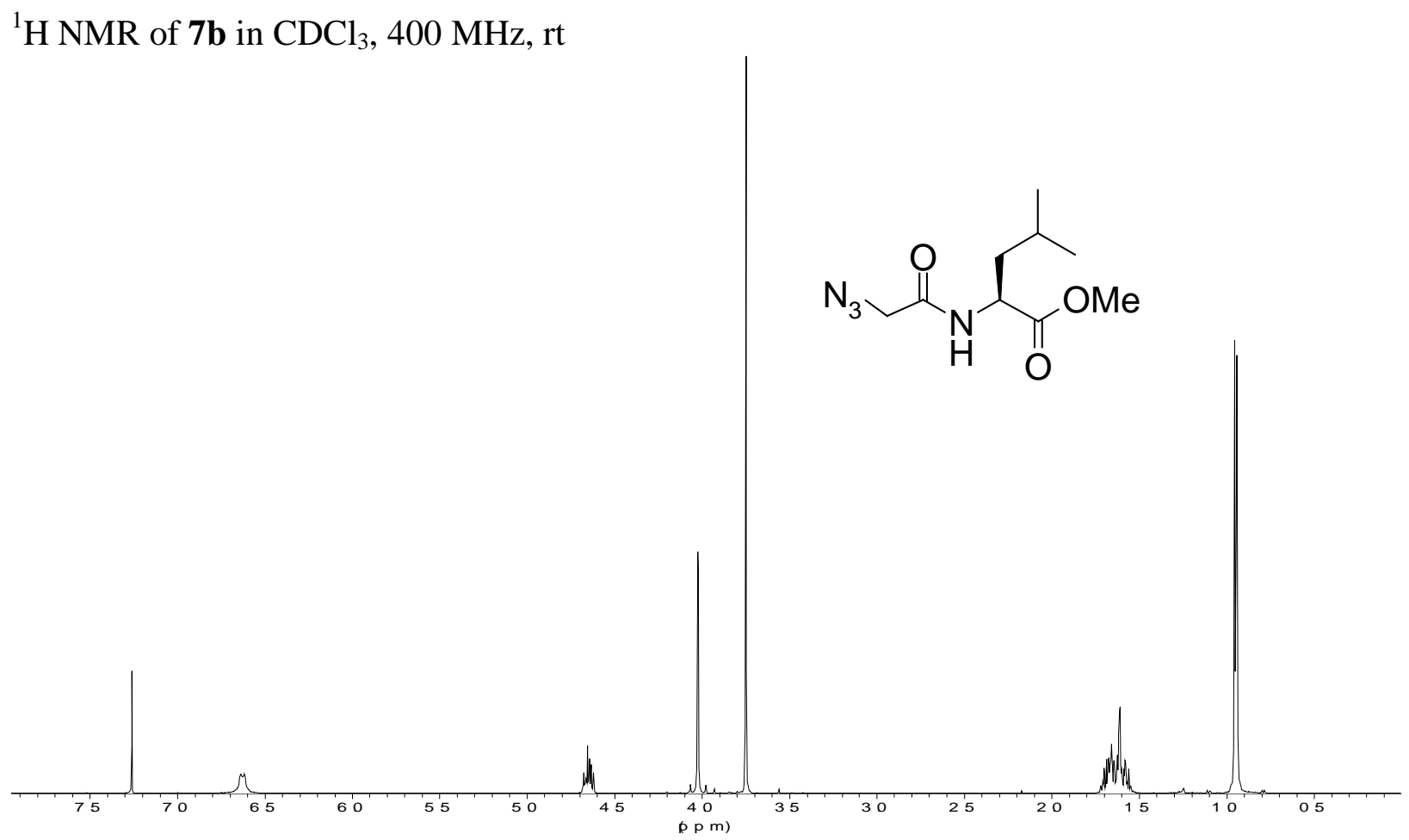

${ }^{13} \mathrm{C}$ NMR of $\mathbf{7 b}$ in $\mathrm{CDCl}_{3}, 62.5 \mathrm{MHz}, \mathrm{rt}$

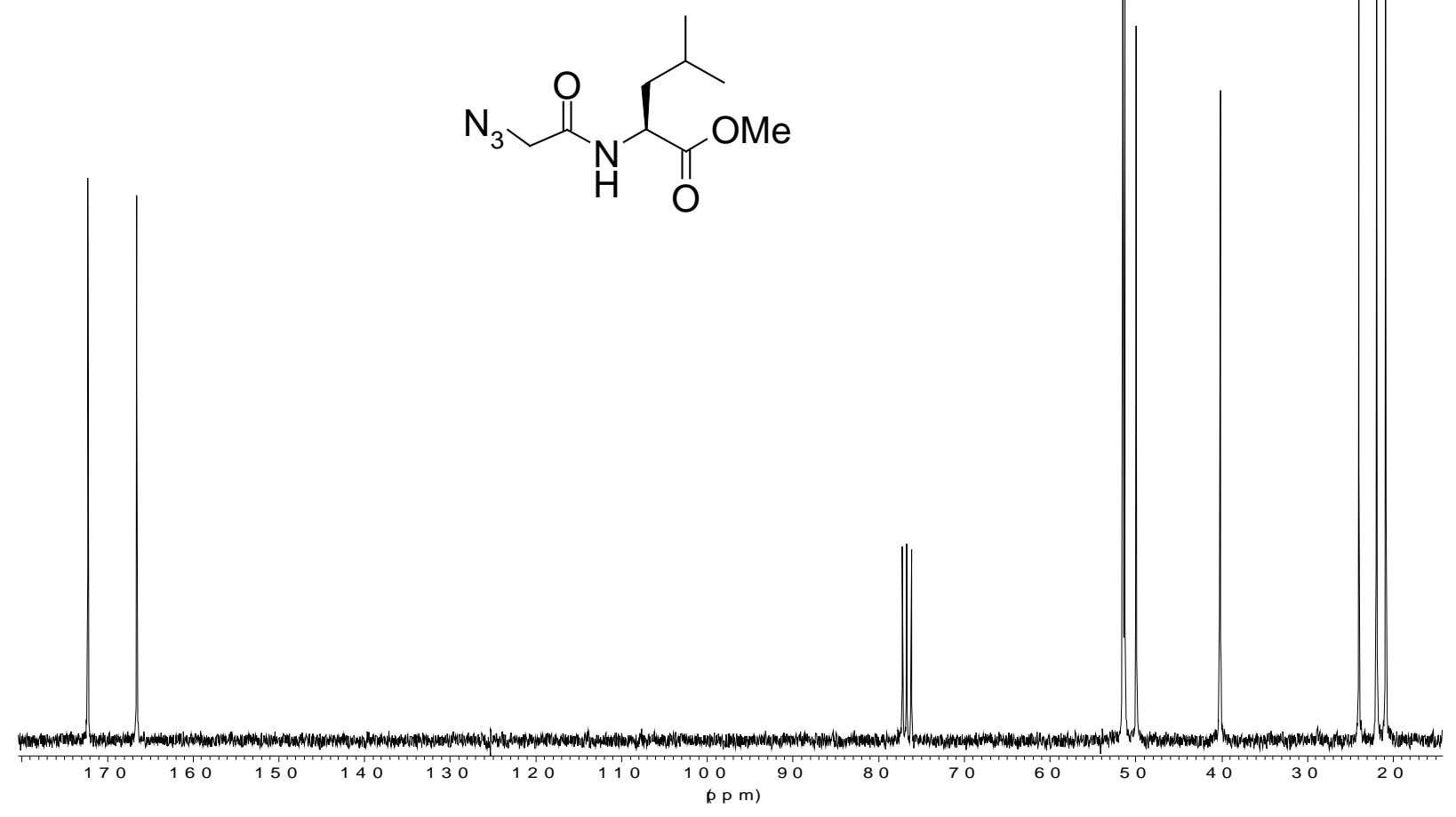


${ }^{13} \mathrm{C}$ pendant NMR of $\mathbf{7 b}$ in $\mathrm{CDCl}_{3}, 100 \mathrm{MHz}, \mathrm{rt}$

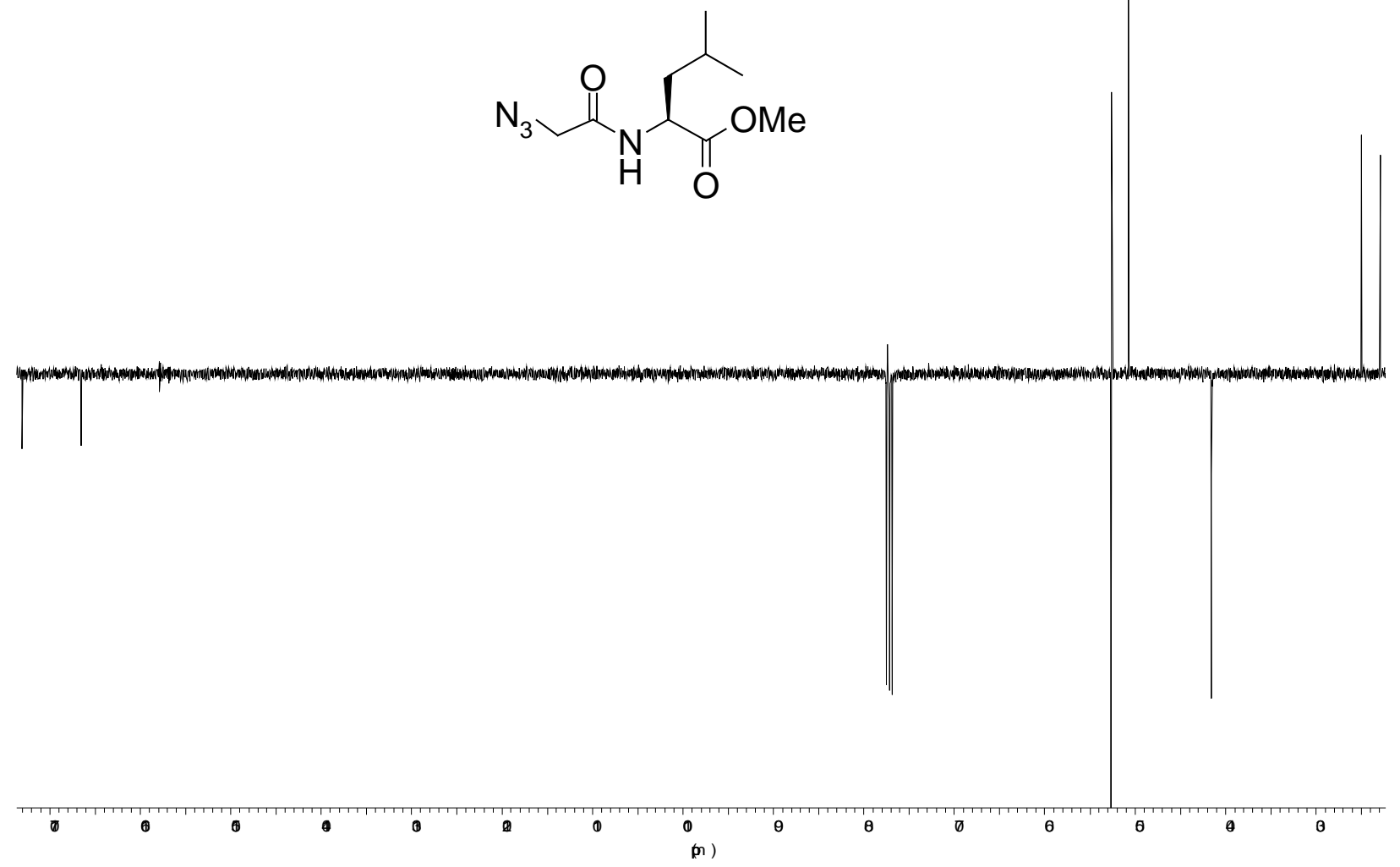

${ }^{1} \mathrm{H}$ NMR of 7c in $\mathrm{CDCl}_{3}, 400 \mathrm{MHz}, \mathrm{rt}$

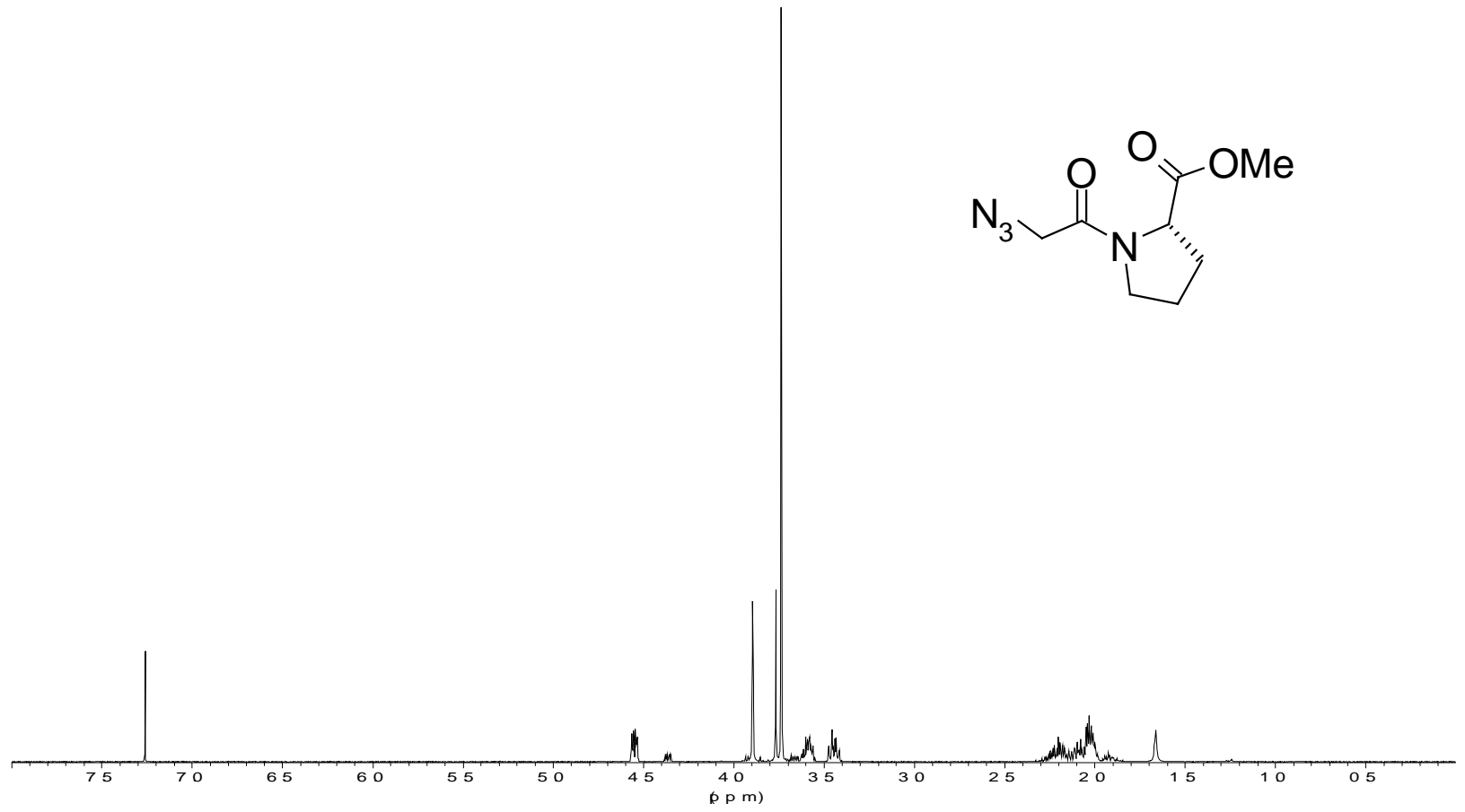


${ }^{13} \mathrm{C}$ NMR of 7c in $\mathrm{CDCl}_{3}, 62.5 \mathrm{MHz}, \mathrm{rt}$

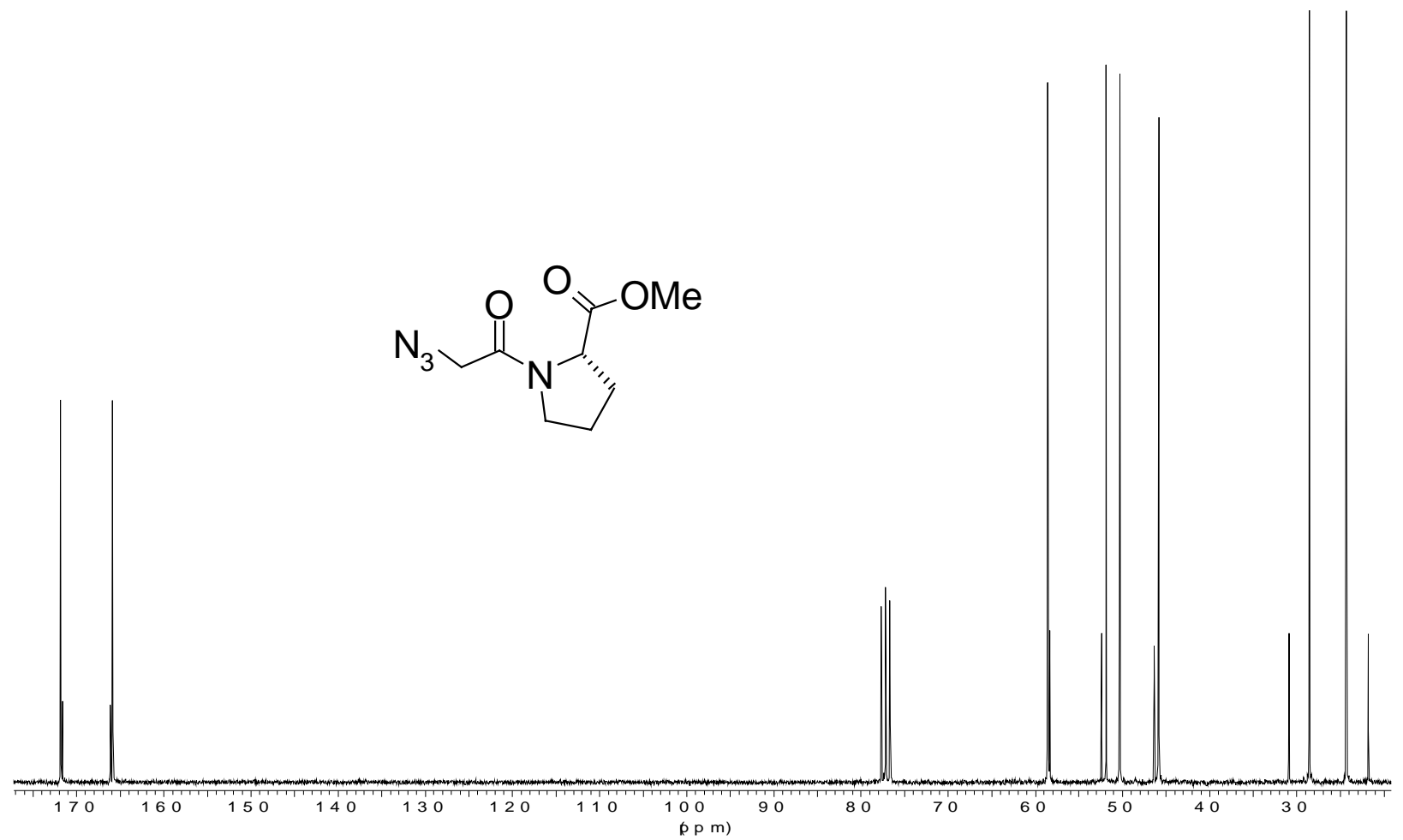

${ }^{13} \mathrm{C}$ pendant $\mathrm{NMR}$ of $7 \mathrm{c}$ in $\mathrm{CDCl}_{3}, 100 \mathrm{MHz}, \mathrm{rt}$

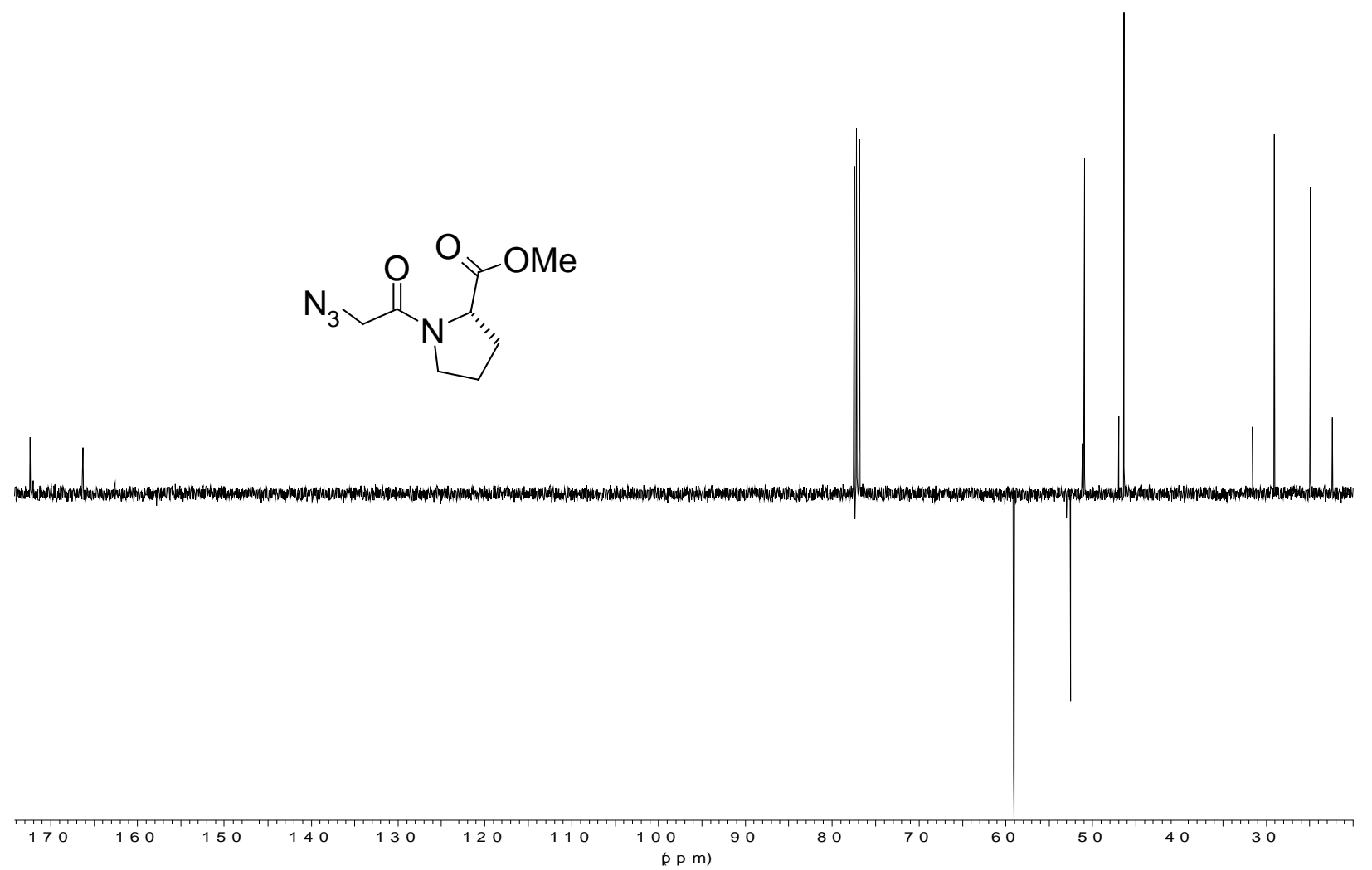


${ }^{1} \mathrm{H}$ NMR of 7d in $\mathrm{CDCl}_{3}, 400 \mathrm{MHz}, \mathrm{rt}$

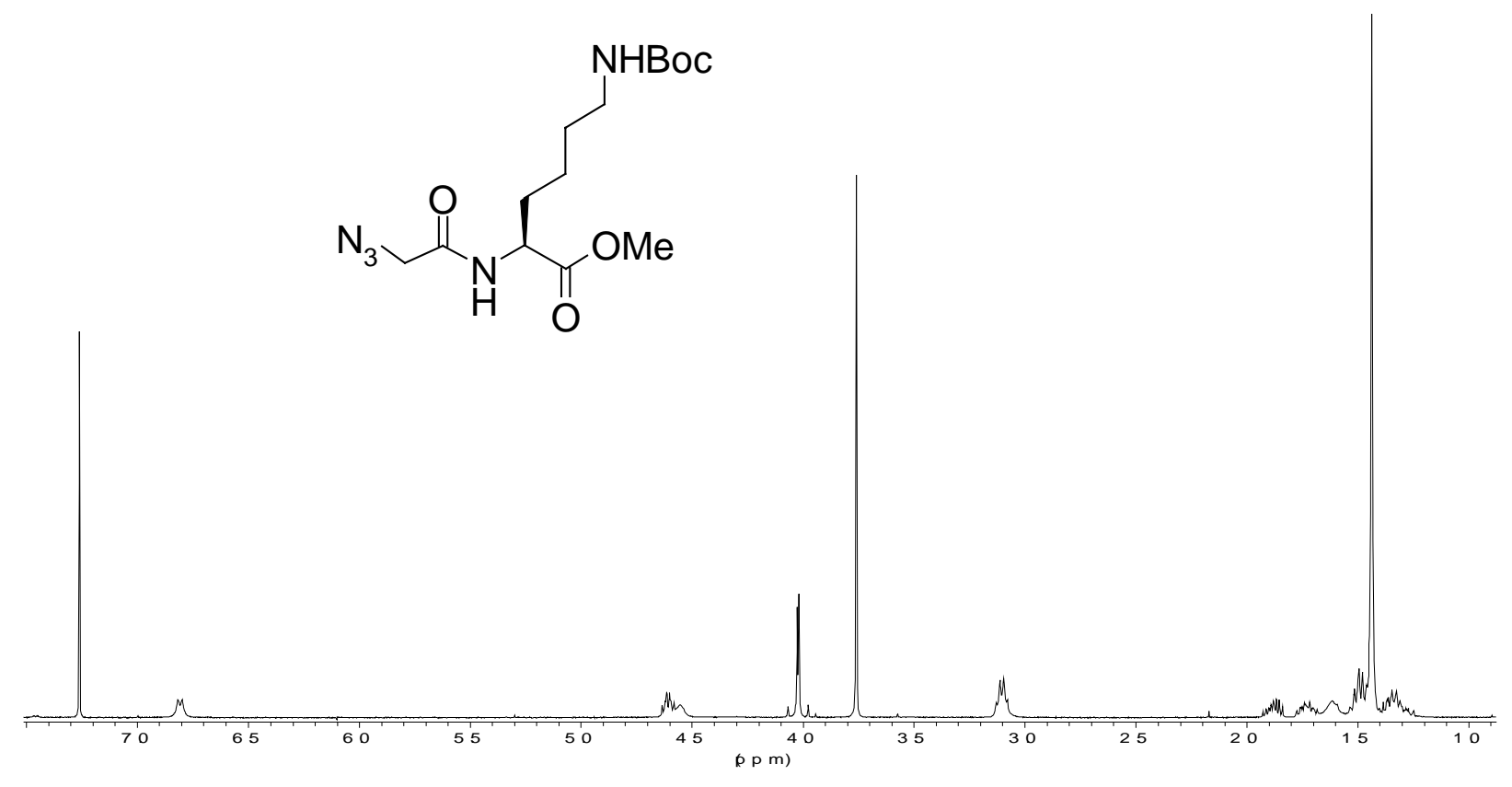

${ }^{13} \mathrm{C}$ NMR of 7d in $\mathrm{CDCl}_{3}, 62.5 \mathrm{MHz}, \mathrm{rt}$

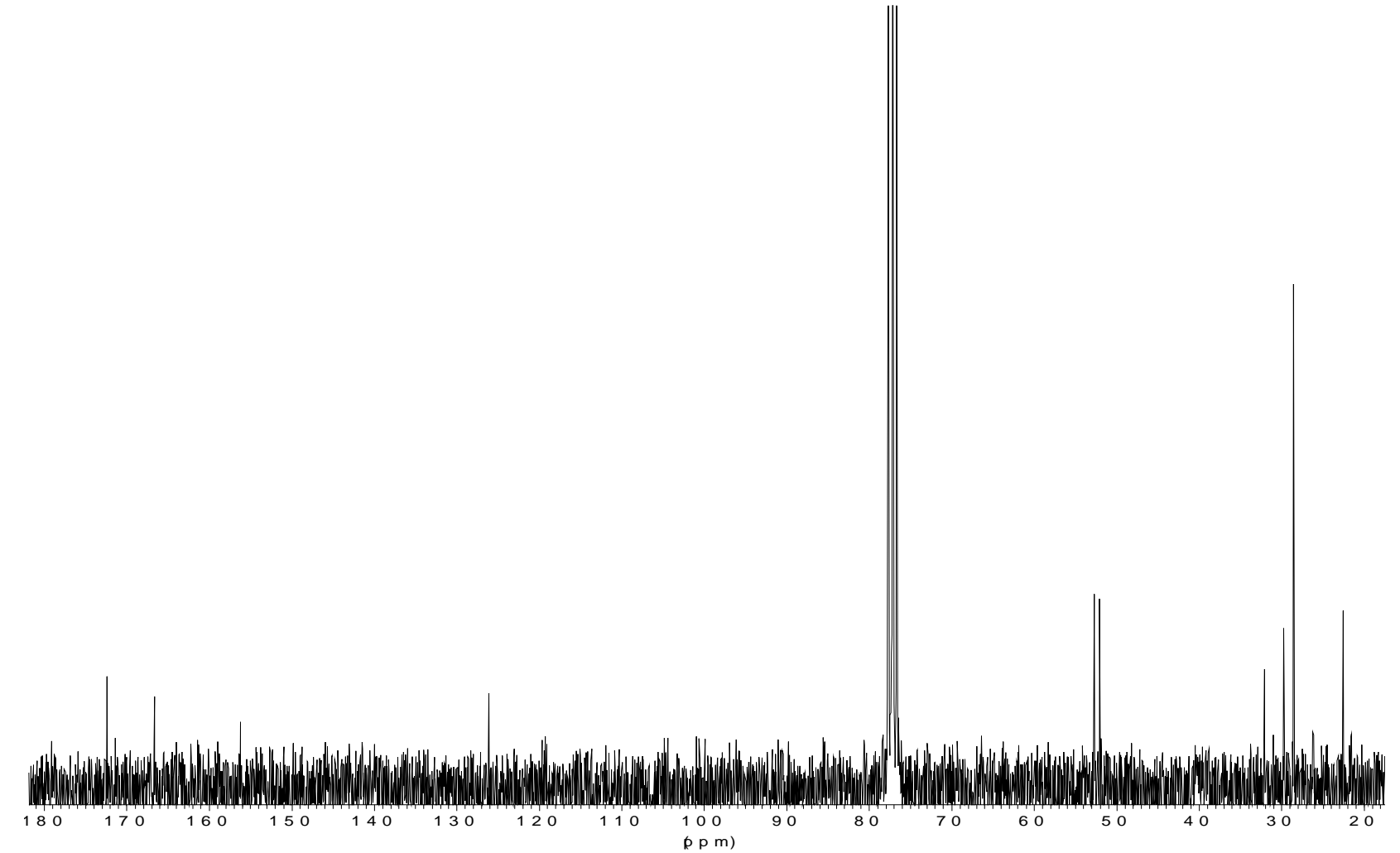


${ }^{13} \mathrm{C}$ pendant $\mathrm{NMR}$ of $7 \mathbf{d}$ in $\mathrm{CDCl}_{3}, 100 \mathrm{MHz}, \mathrm{rt}$

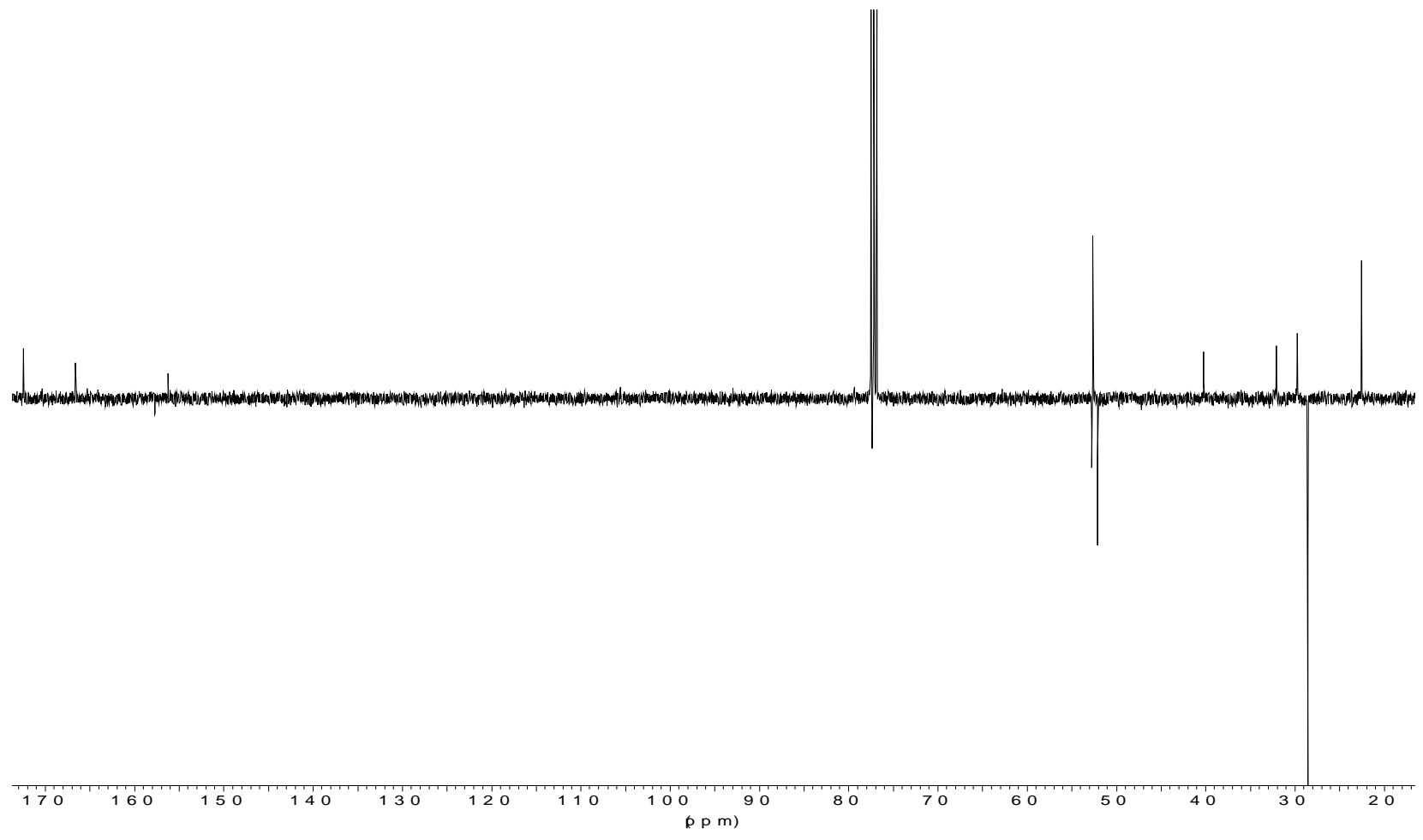

${ }^{1} \mathrm{H}$ NMR of 8 in $\mathrm{CDCl}_{3}, 400 \mathrm{MHz}, \mathrm{rt}$
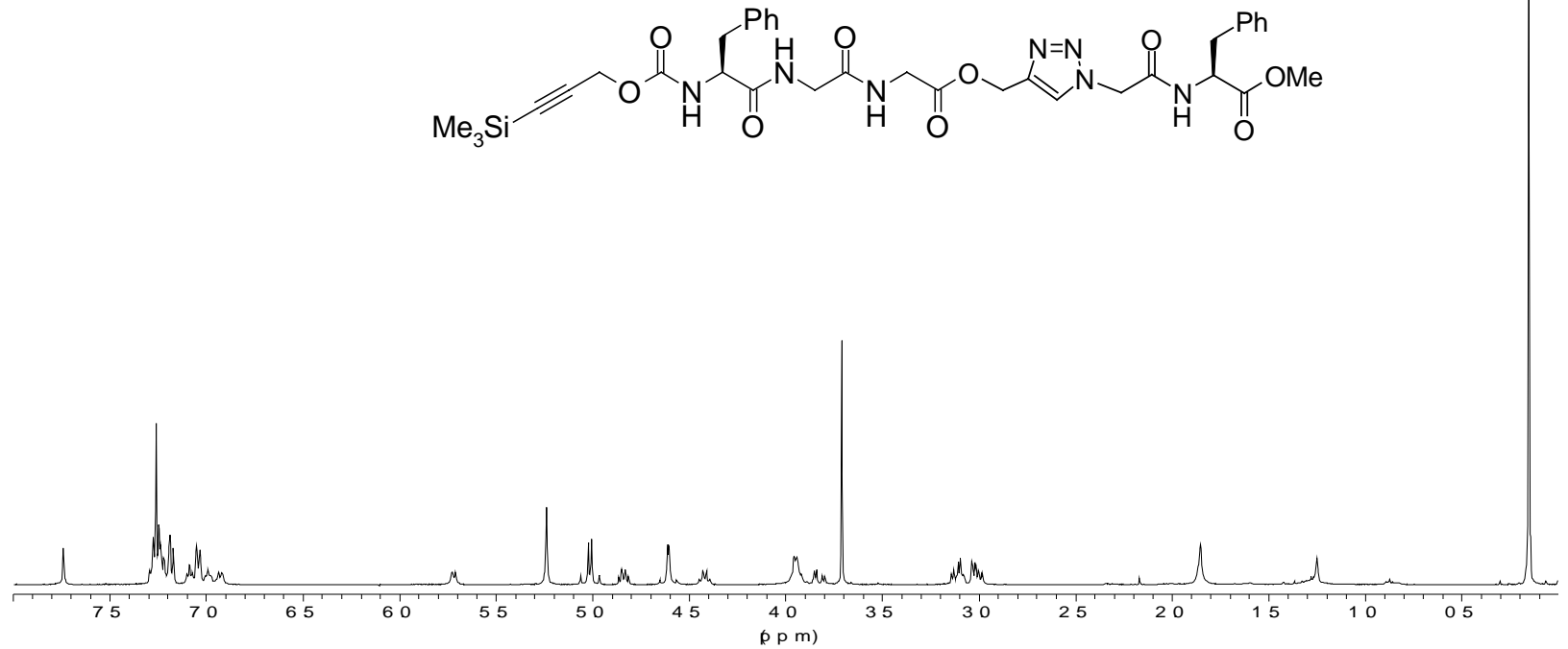
${ }^{13} \mathrm{C}$ NMR of 8 in $\mathrm{CDCl}_{3}, 125 \mathrm{MHz}, \mathrm{rt}$

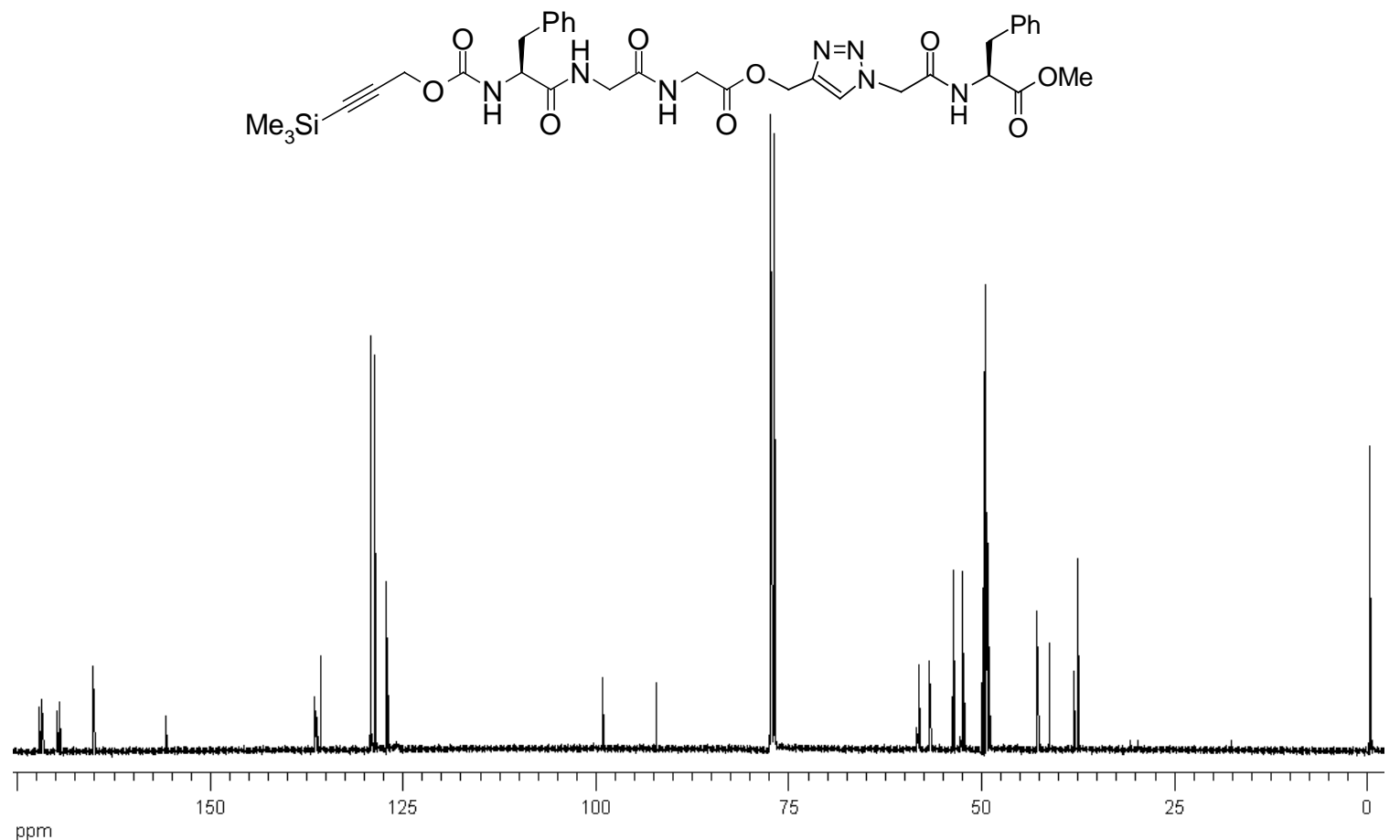

${ }^{13} \mathrm{C}$ pendant $\mathrm{NMR}$ of 8 in $\mathrm{CDCl}_{3}, 100 \mathrm{MHz}, \mathrm{rt}$

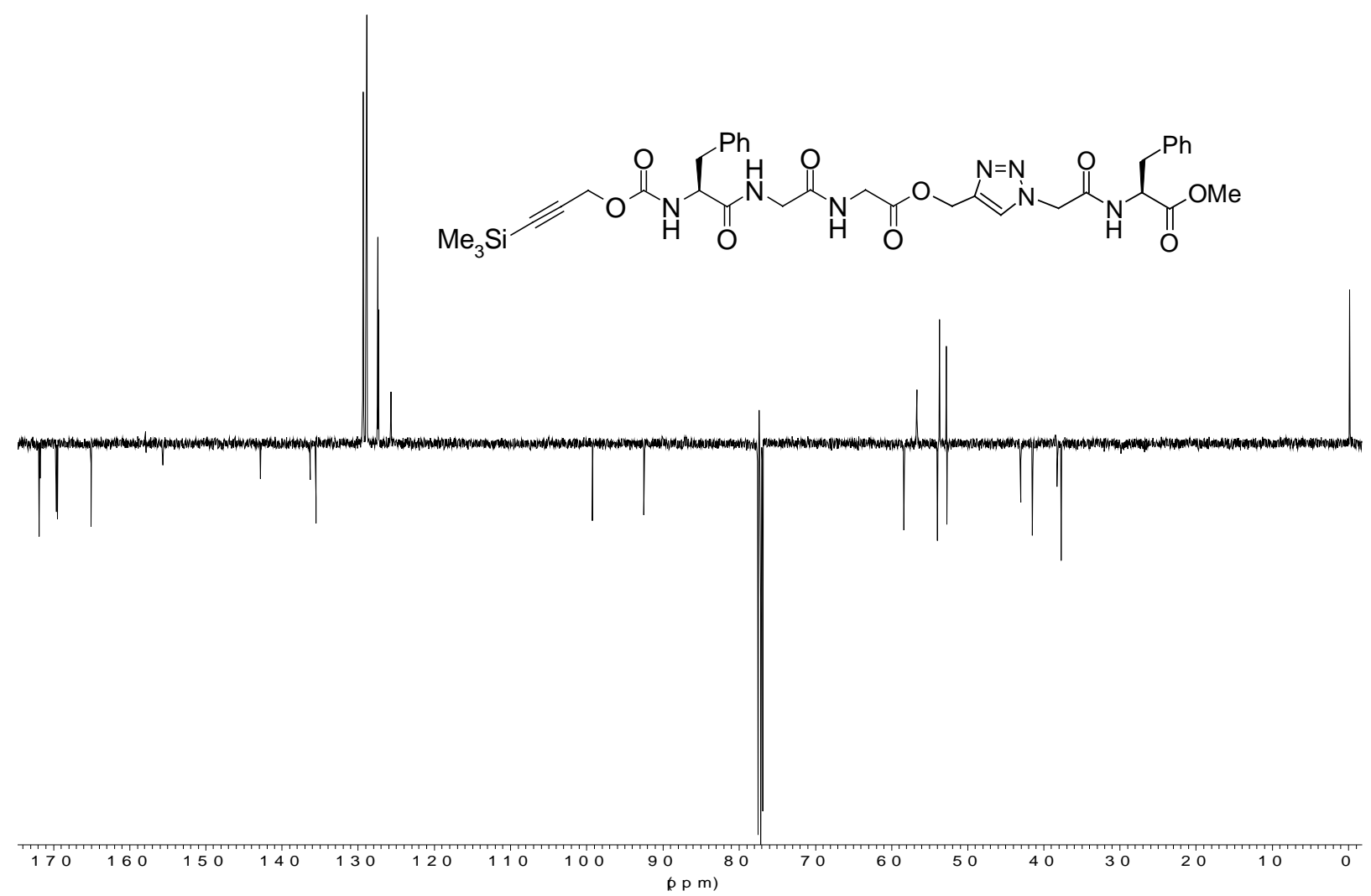


HPLC chromatogram of $\mathbf{8}$, gradient B

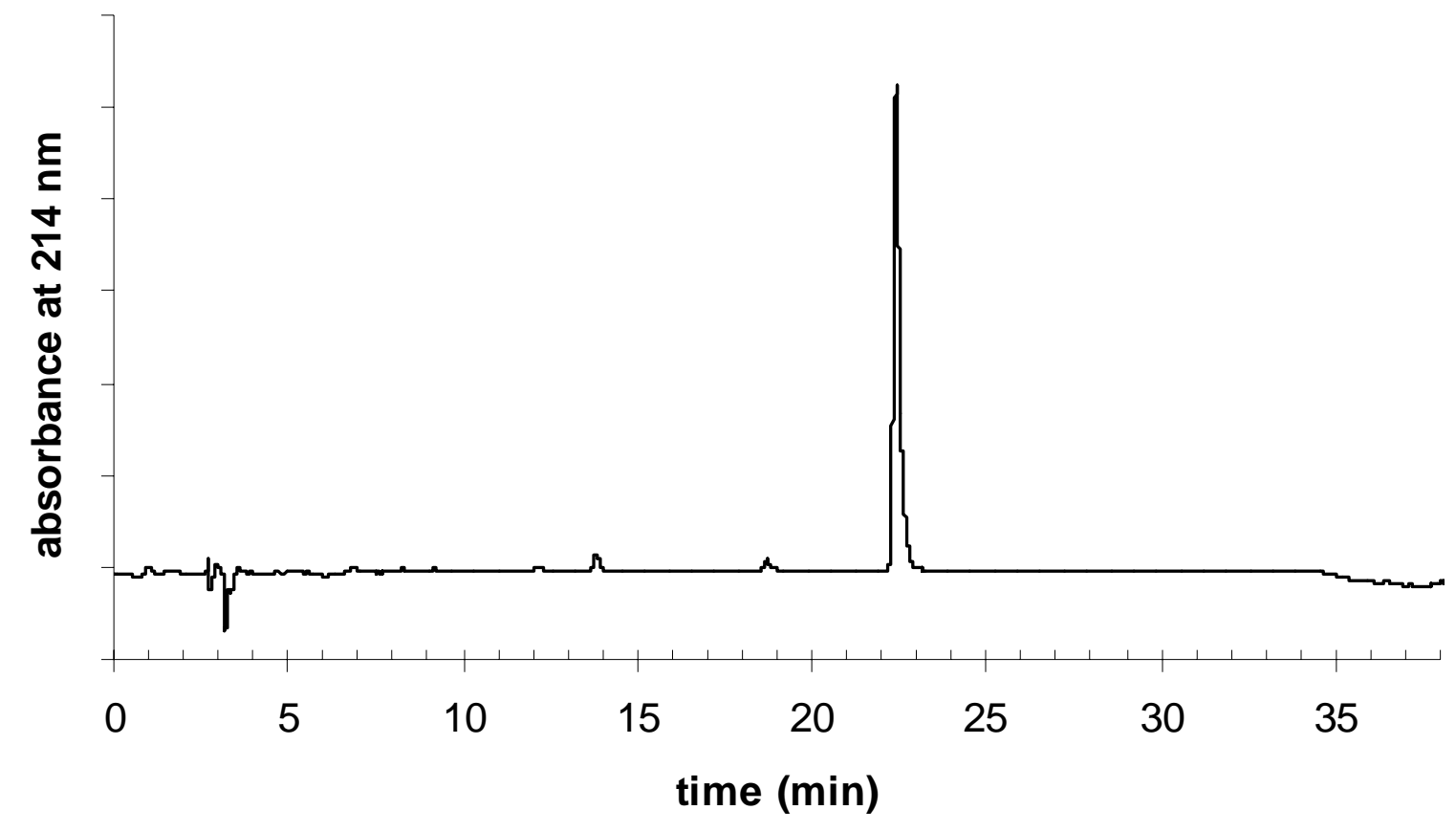

${ }^{1} \mathrm{H}$ NMR of 9 in $\mathrm{CDCl}_{3}: \mathrm{CD}_{3} \mathrm{OD} 98: 2,400 \mathrm{MHz}, \mathrm{rt}$

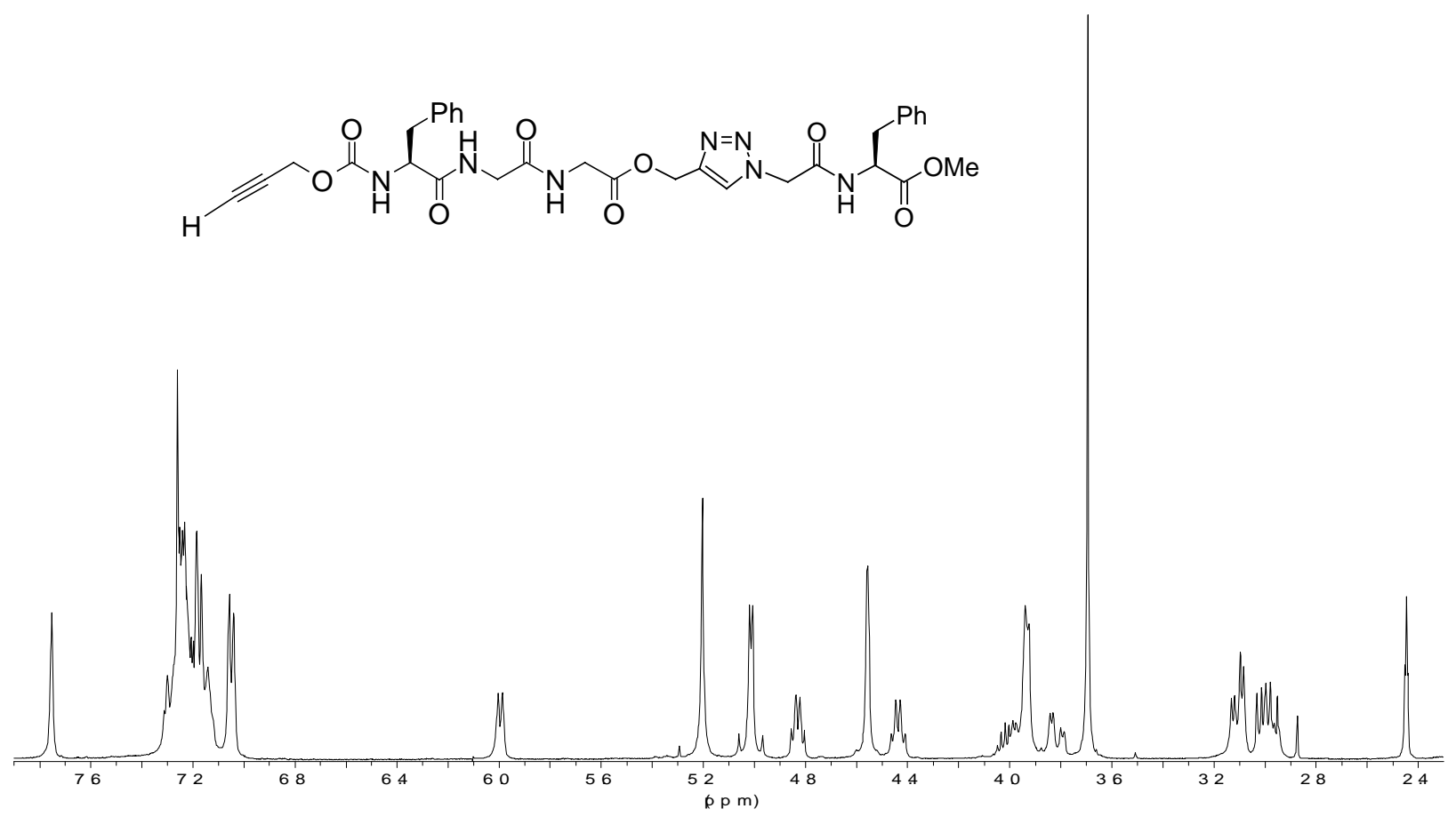


${ }^{13} \mathrm{C}$ pendant $\mathrm{NMR}$ of 9 in $\mathrm{CDCl}_{3}: \mathrm{CD}_{3} \mathrm{OD} 98: 2,100 \mathrm{MHz}, \mathrm{rt}$

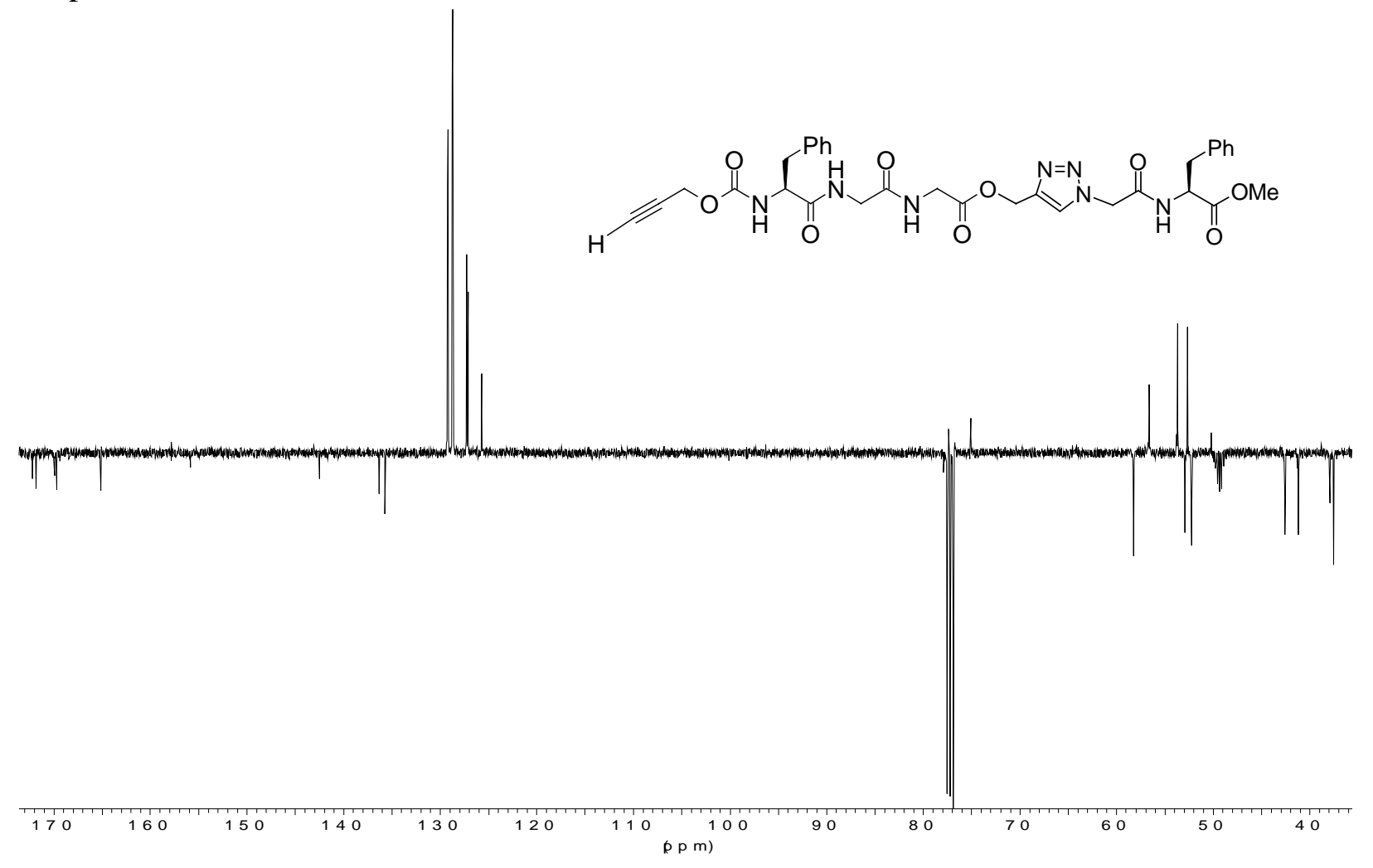

${ }^{1} \mathrm{H}$ NMR of 10a in $\mathrm{CDCl}_{3} / \mathrm{CD}_{3} \mathrm{OD} 8: 2,400 \mathrm{MHz}$, rt

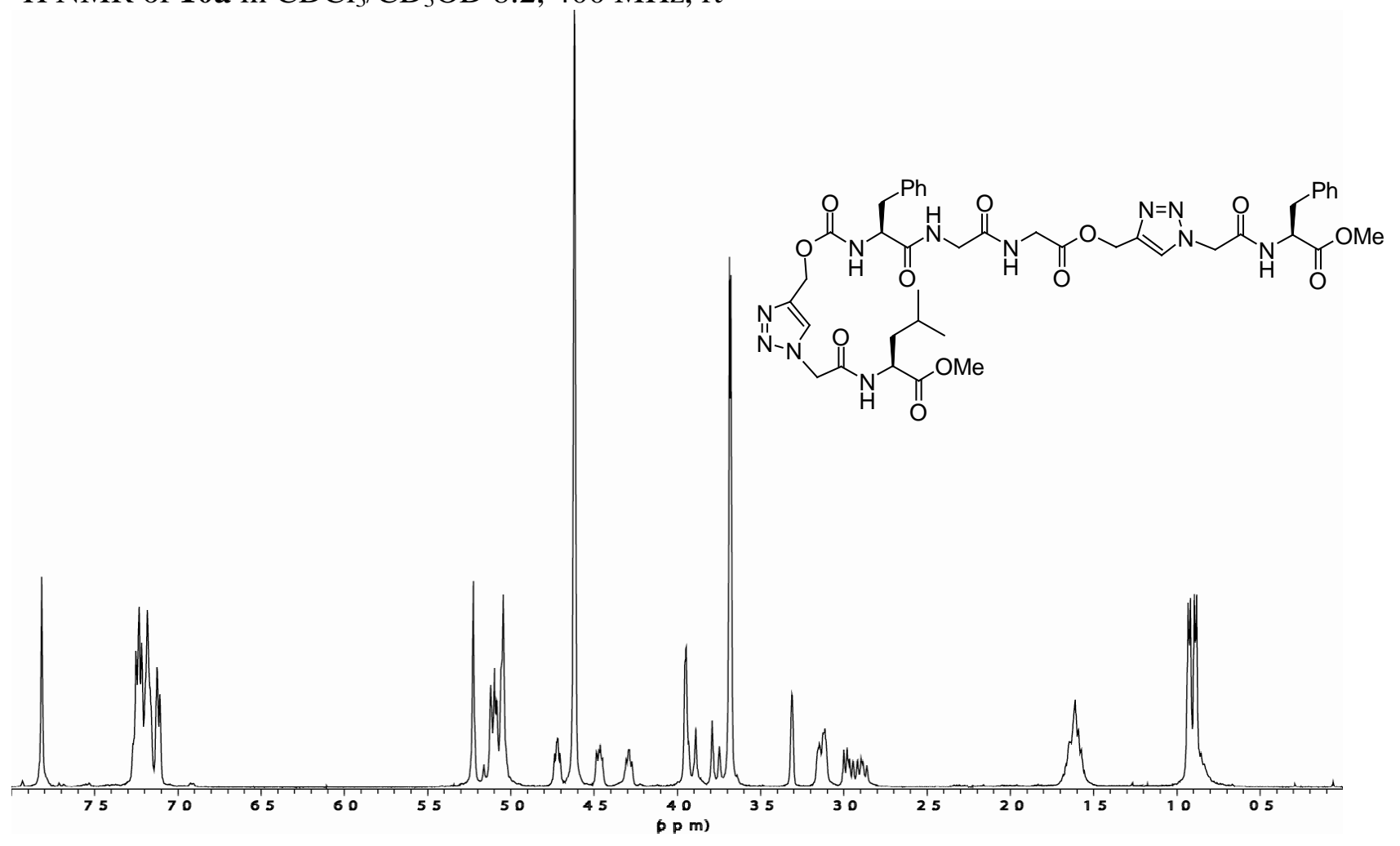


${ }^{13} \mathrm{C}$ NMR of 10a in $\mathrm{CDCl}_{3} / \mathrm{CD}_{3} \mathrm{OD} 8: 2,100 \mathrm{MHz}, \mathrm{rt}$<smiles>COC(=O)C(Cc1ccccc1)NC(=O)Cn1cc(COC(=O)NC(Cc2ccccc2)C(=O)NCC(=O)NCC(=O)OCc2cn(CC(=O)NC(CC(C)O)C(=O)OC)nn2)nn1</smiles>

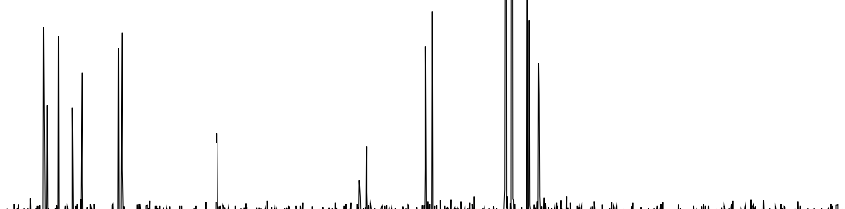

$170160 \quad 150 \quad 140$

HPLC chromatogram of $\mathbf{1 0 a}$, gradient A

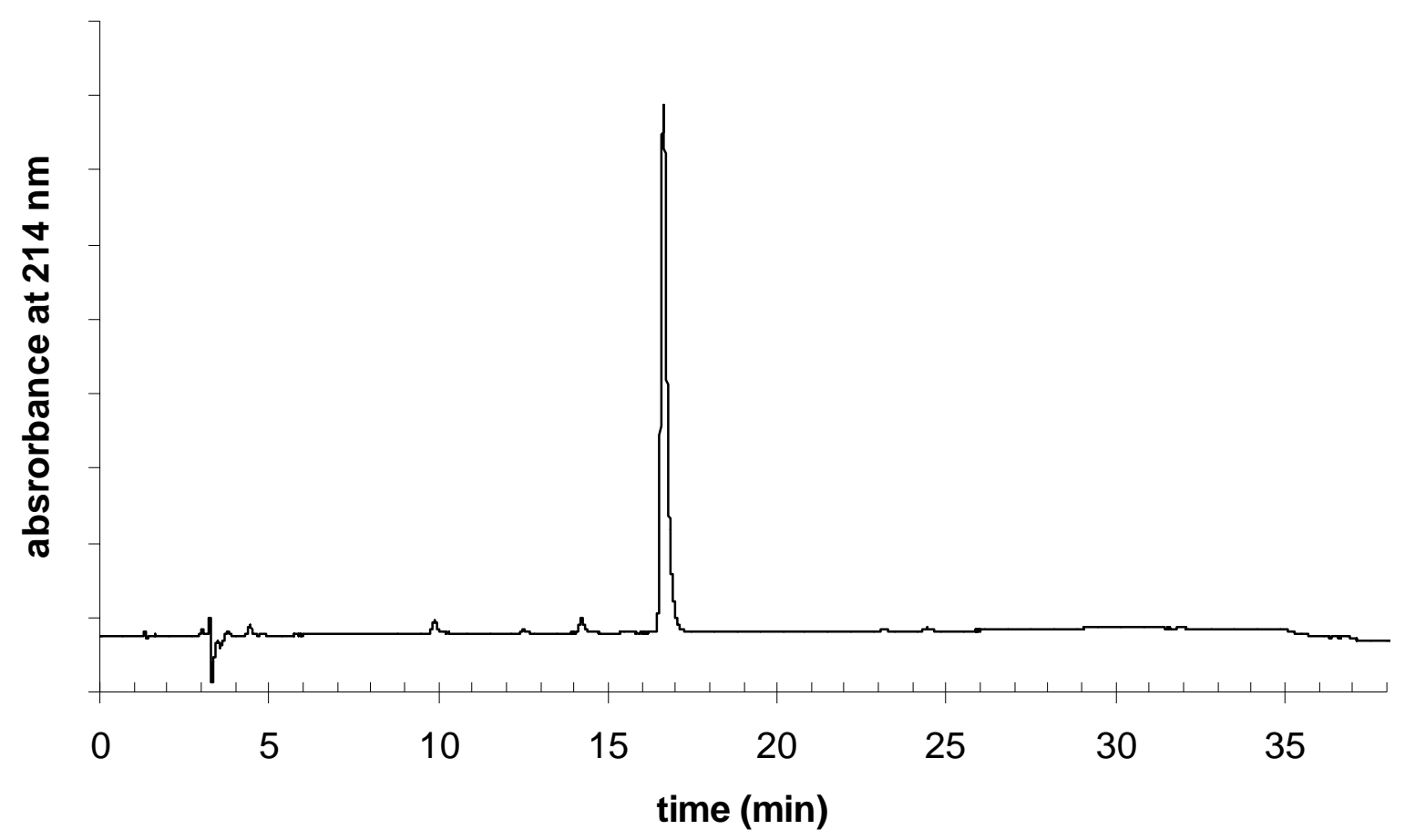


${ }^{1} \mathrm{H}$ NMR of 10b in $\mathrm{CDCl}_{3} / \mathrm{CD}_{3} \mathrm{OD}$ 95:5, $400 \mathrm{MHz}, \mathrm{rt}$<smiles>COC(=O)C(Cc1ccccc1)NC(=O)Cn1cc(COC(=O)CNC(=O)CNC(=O)C(Cc2ccccc2)NC(=O)OCc2cn(CC(=O)N3CCCC3C(=O)OC)nn2)nn1</smiles>

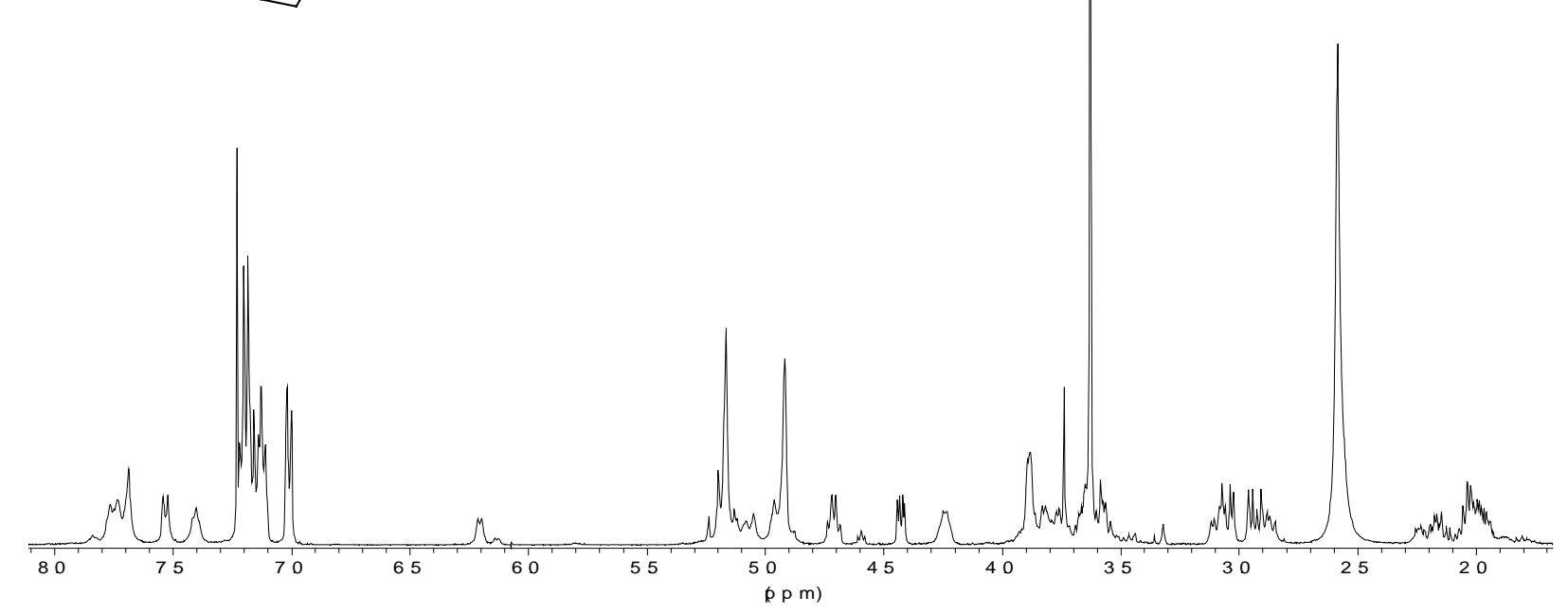

${ }^{13} \mathrm{C}$ NMR of 10b in $\mathrm{CDCl}_{3} / \mathrm{CD}_{3} \mathrm{OD} 95: 5,125 \mathrm{MHz}$, rt

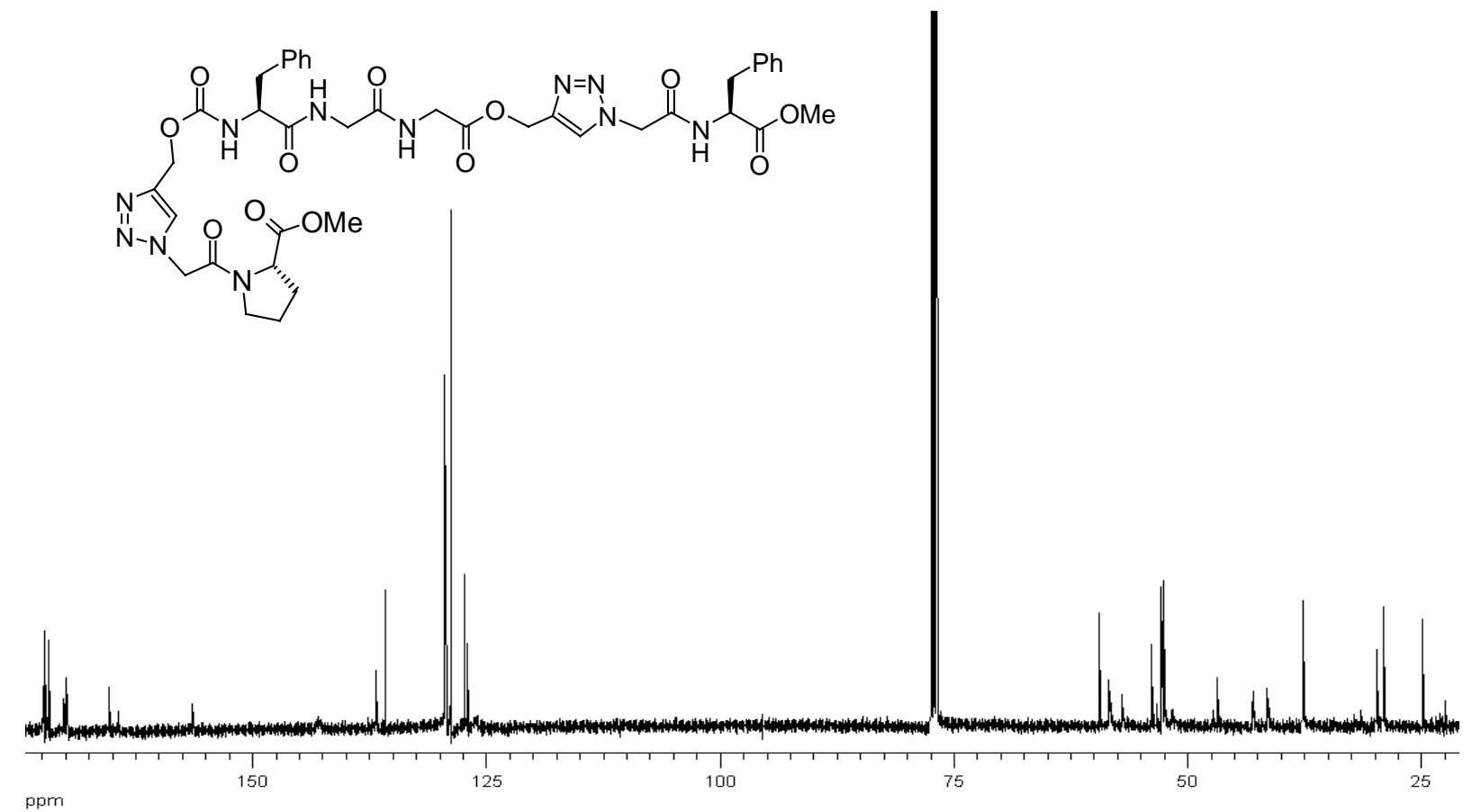


HPLC chromatogram of $\mathbf{1 0 b}$, gradient A

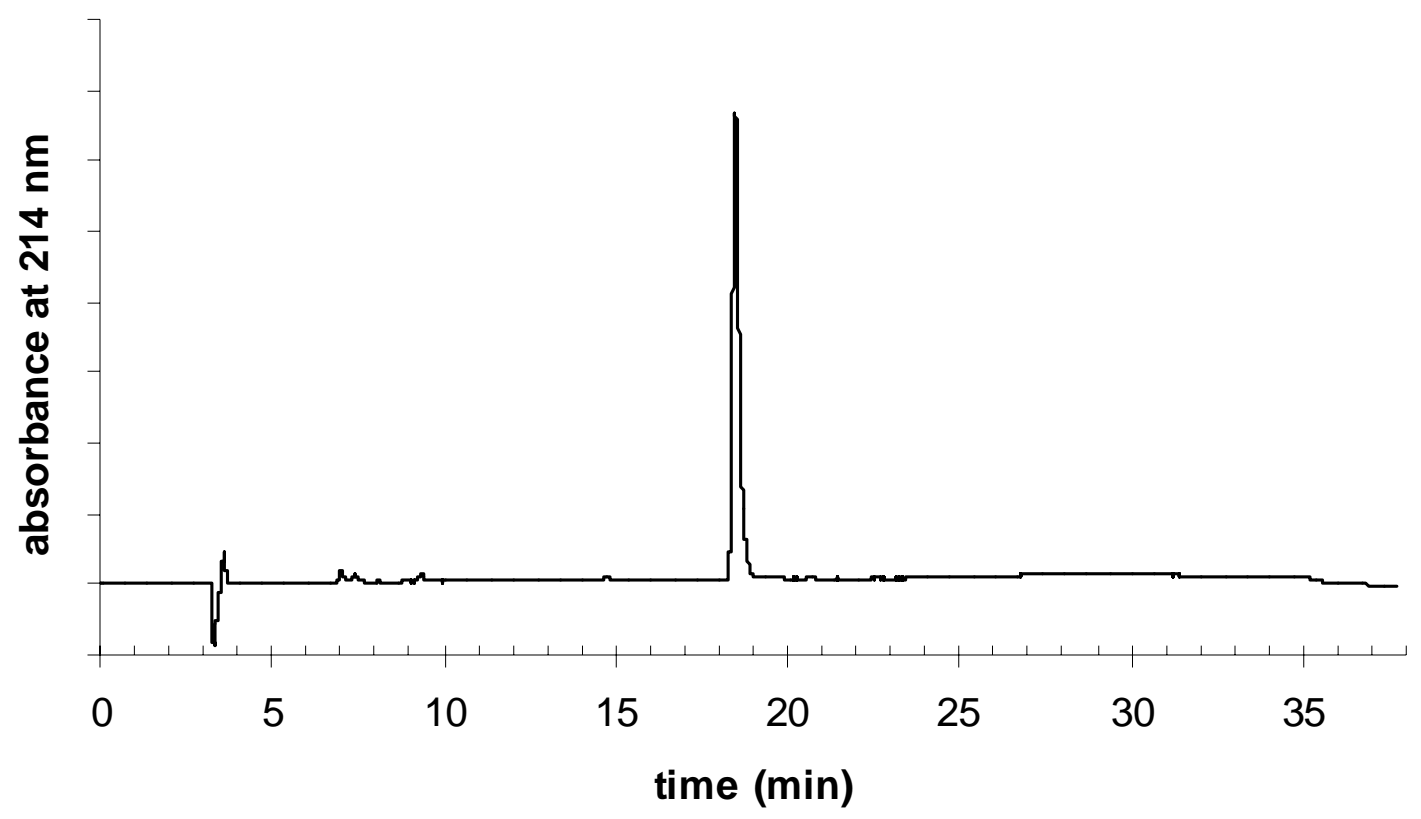

${ }^{1} \mathrm{H}$ NMR of 10c in $\mathrm{CDCl}_{3} / \mathrm{CD}_{3} \mathrm{OD} 9 / 1,500 \mathrm{MHz}, \mathrm{rt}$<smiles>COC(=O)C(CCCNC(=O)OC(C)(C)C)NCC(=O)OCC(=O)NCC(=O)NC(Cc1ccccc1)C(=O)NC(Cc1cn(CC(=O)NC(Cc2ccccc2)C(=O)OC)nn1)C(=O)NC(C)(C)C</smiles>

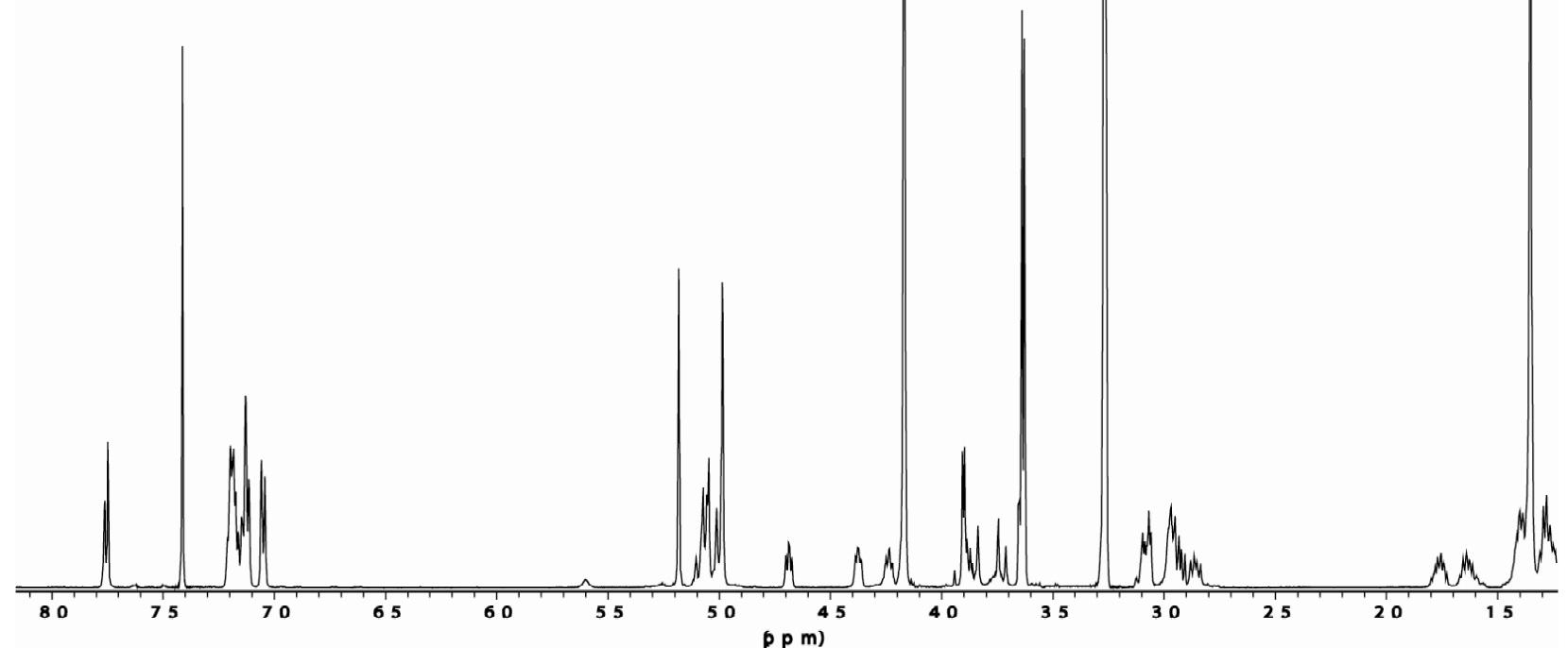


${ }^{13} \mathrm{C}$ NMR of 10c in $\mathrm{CDCl}_{3} / \mathrm{CD}_{3} \mathrm{OD} 9 / 1,125 \mathrm{MHz}, \mathrm{rt}$<smiles>COC(=O)C(CCCNC(=O)OC(C)(C)C)NCC(=O)NCC(=O)OCc1cn(CC(=O)NC(Cc2ccccc2)C(=O)OC)nn1</smiles>

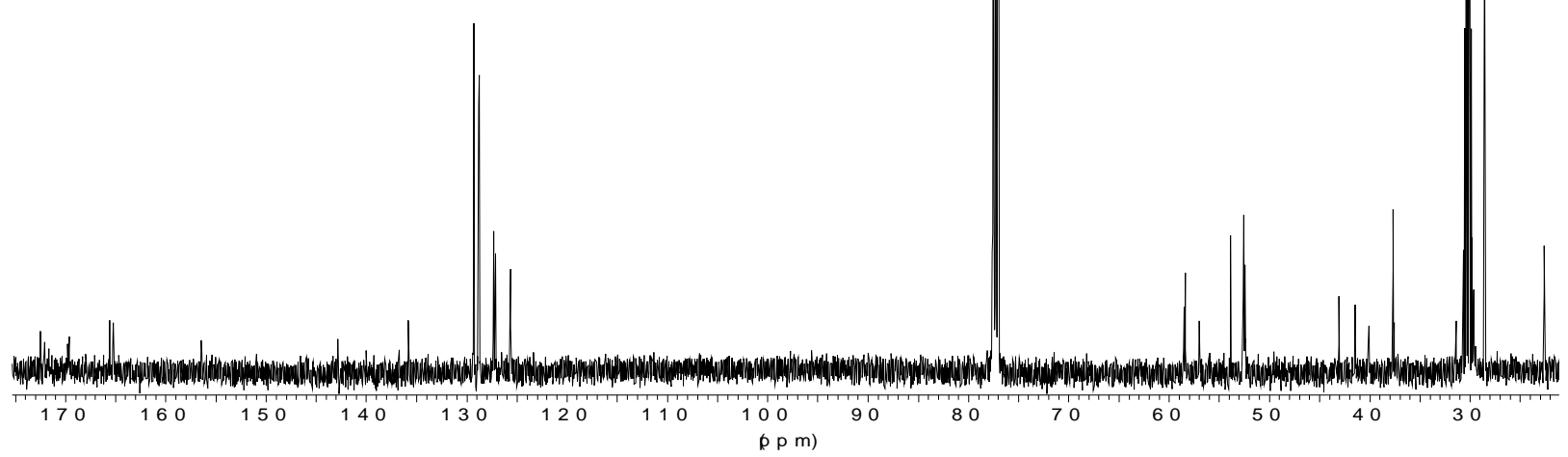

HPLC chromatogram of $\mathbf{1 0 c}$, gradient B

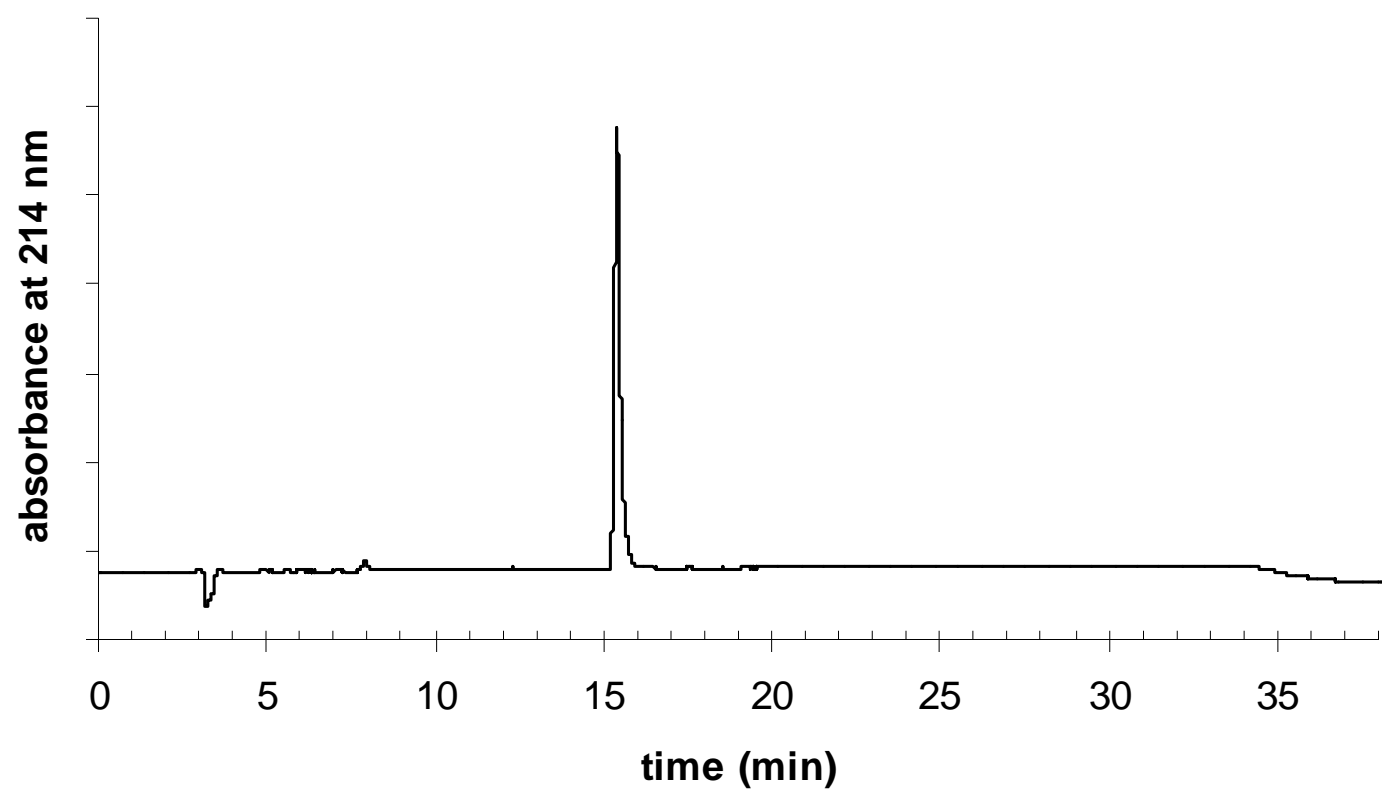

